\title{
Future Smart Cities: Requirements, Emerging Technologies, Applications, Challenges, and Future Aspects
}

\author{
Faisal Shahzad, Saif ur Rehman, Abdul Rehman Javed, Zunera Jalil, Yousaf Bin Zikria
}

\begin{abstract}
Future smart cities are the key to fulfill the evergrowing demands of citizens. Information and communication advancements will empower better administration of accessible resources. The eventual fate of the world lies in its urban environment. A more significant part of the total populace lives in urban areas, and that number is growing. This fast influx of individuals creates possibility, yet it additionally causes challenges. Creating sustainable, reasonable space in the world's steadily extending cities is tested confronting governments worldwide. The model of the Smart Cities rise, where the rights and well-being of the smart city citizens are assured, the industry is in action, and the assessment of urban planning from an environmental point of view. This paper presents a survey on the analysis of future technologies and requirements for future Smart Cities. We provide extensive research to identify and inspect the latest technology advancements, which acts as the foundation stone of the upcoming robust era. Such technologies include deep learning (DL), machine learning (ML), internet of things (IoT), mobile computing, big data, blockchain, sixth-generation (6G) networks, WiFi-7, industry 5.0, robotic systems, heating ventilation, and air conditioning (HVAC), digital forensic, industrial control systems, connected and automated vehicles (CAVs), electric vehicles, product recycling, flying Cars, pantry backup, calamity backup and vital integration of cybersecurity to keep the user concerns secured. We provide a detailed review of the existing future smart cities application frameworks. Furthermore, we discuss various technological challenges of future smart cities. Finally, we identify the future dimensions of smart cities to develop smart cities with the precedence of smart living.
\end{abstract}

Index Terms-Survey, State-of-the-Art, Future Cities, Smart cities , Internet of Things, Big Data, Cloud computing, Intelligent sensors, Transportation, Data Mining, smart education, smart health, smart mobility, urban modeling, Real-time systems, Technologies, Applications, Challenges, Best Practices

\section{INTRODUCTION}

Internet and its allied technologies are duly penetrating in every field. The benefits of technology incorporation in everyday life have now surpassed from impacting the individual level and giving benefits to masses at the community and metropolitan level. The critical component of

Faisal Shahzad is with the Department of Cyber Security, Air University, Islamabad, Pakistan (e-mail: faisal.rwp@gmail.com).

Saif ur Rehman is with the Department of Computer Science, Air University, Islamabad, Pakistan (e-mail: 181065@students.au.edu.pk).

Abdul Rehman Javed is with the Department of Cyber Security, Air University, Islamabad, Pakistan (e-mail: abdulrehman.cs@au.edu.pk).

Zunera Jalil is with the Department of Cyber Security, Air University, Islamabad, Pakistan (e-mail: zunera.jalil@mail.au.edu.pk).

Yousaf Bin Zikria (Corresponding Author) is with the Department of Information and Communication Engineering, College of Engineering, Yeungnam University, Gyeongsan-Si 38541, South Korea (e-mail: yousafbinzikria@ynu.ac.kr). these new internet-enabled technologies is IoT devices which made reality to make us live in smart cities [1]. These IoT devices revolutionize human-computer interaction (HCI) to bring the paradigm shift and open new technology integration methods within everyday life. Now the HCI technology is more human-centric than computer-centric as in the past [2]. The sustainability and coherence in the HCI in the past decade results in increased dimensions where $\mathrm{HCI}$ is now utilized in a more effective way [3].

To reap the benefits of smart governance and management, countries started incorporating innovative techniques and technologies to manage better and govern the affairs of their cities [4]. For having a truly smart city, almost all required aspects of management and governance of that city need to incorporate these technical advancements within the allied processes. A city transformation is required at the design level to make a city truly smart which is a substantially primary task and can positively impact socially, economically, humanly, and environmentally.

Many cities in the developed world are already on the move to smart cities technology implementation. These cities include (but not limited to) London, Stockholm, Dubai, New York, Barcelona, Hong Kong, Amsterdam, Singapore, Tokyo, Paris, and Copenhagen [5]. A conceptual model of a smart city is also included in ISO standard library known as ISO/IEC 30182:2017(en) Smart city concept model. It guides establishing a model for data interoperability. It is seen as a first step of the organization towards the standardization of the various aspects of smart cities in the coming future [6].

Motivation: Smart cities are composed of information and communication technologies (ICT) and the IoT to increase operational efficiency, improve both the quality of government services and citizen welfare, develop, deploy, and promote sustainable development practices to fulfill the ever-growing demands of citizens. Information and communication advancements empower better administration of accessible resources. Development of the technology has a major reason to bring ease and innovation into daily human life. Moreover, the research conducted over the emerging technologies also intends to bring efficiency to the present solutions. Increasing the efficiency of these technologies can make them eco-friendly, more productive, and agile. The digital revolution proved to be key to success wherever applied-keeping in view the easiness and efficiency in operation. In contrast, making an informed decision based on data and facts can make it the key interacting factor in any field. The same holds for the Smart 
City arena. Thus, this makes a major cause of researching the improvement of upcoming technology to better human life. Envisioning the future aspects of smart cities through the current scenarios opens the door to new research. It helps the researchers develop a wholly new framework of the smart cities to view the interacting aspect mentioned in this paper. This paper can be regarded as the overview of the technical environment, which decides the compatibility and functionalities of different technologies and their outcome when those technologies work together. To our knowledge, this is the first approach of looking into the Future Smart Cities as a System of Systems and discussion on the various interacting aspects of smart cities in a research-oriented way. The taxonomy presented in this paper covers the future smart cities on the angle of 360 degrees, i.e., try to encapsulate all the major aspects/systems interacting with each other to become a system we know as Smart City.

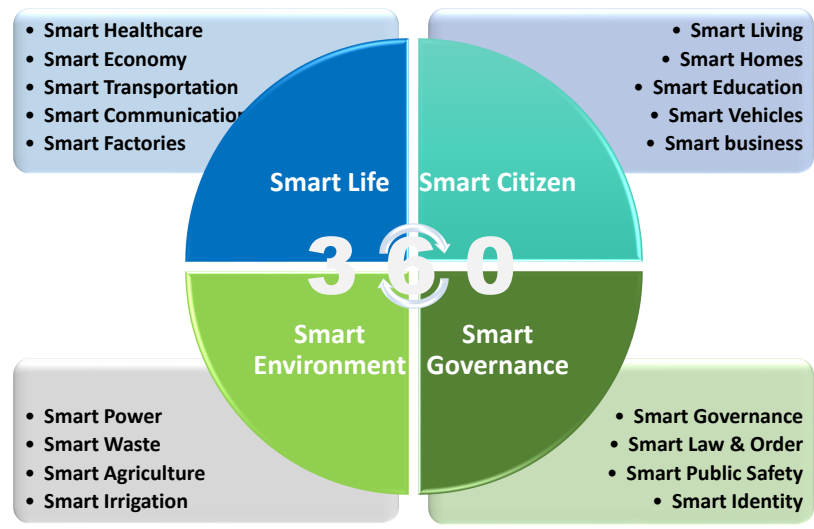

Fig. 1: 360 Degrees Of Smartness in Smart City - A System Of Systems Approach

A 360-degree technological coverage is required to make a city smart in true aspects. Fortunately, in the fourth industrial revolution, the area of communication, compute, and storage observed a drastic reduction in hardware costs, which made this technology integration possible and cost-effective to reach the common person level. This paper discusses 360-degree coverage requirements, challenges, and implementation strategies briefly to make them a baseline standard for future smart cities. Figure 1 presents 360-degree coverage of smart cities.

Figure 2 depicts the taxonomy diagram of the proposed survey. The taxonomy includes seven significant aspects of advancing and developing a smart city, including 24 critical sub-sections of research areas necessary for developing the future smart city.

Contribution: This paper targets to present an in-depth survey and analysis of future technologies for Smart cities. As smart cities' concept is transforming with the advances in complementary technologies, there is a need to accumulate the works in these different fields and create a unified repository. We can summarize our contributions as follows.
1) We present an overview of the future smart cities, their robust capabilities to revolutionize the way of living, and smart solutions to various problems. The requirements to achieve the proposed theory are also highlighted by studying the current state-of-the-art researches and applications developed for future smart cities to date.

2) We provide an analysis of future smart cities' characteristics based on state-of-the-art technologies, including AI (AI), IoT, and cybersecurity. Such technologies include DL, ML, IoT, mobile computing, big data, blockchain, 6G networks, WiFi-7, industry 5.0, robotic systems, HVAC, digital forensic, industrial control systems, connected and CAVs, electric vehicles, product recycling, flying Cars, pantry backup, calamity backup and vital integration of cybersecurity to keep the user concerns secured.

3) The core contribution of this extensive research identifies and inspects the latest advancements of technology which acts as the foundation stone of the upcoming robust era. Such technologies include deep and ML, mobile computing, big data, blockchain, and vital cybersecurity integration to keep the user concerns secured.

4) We analyze the most feasible frameworks and their proficiency that combines multiple technologies, which can ultimately result in the efficient implementation of procedures required to develop future smart cities.

5) Next, we provide technology challenges identified in the literature, and finally, the future directions are identified to develop smart cities further. Future research directions based on $6 \mathrm{G}$ networks, WIFI 7, Big data 2.0, industry 5.0 , and the internet of nano things (IoNT) are also introduced.

Paper Organization: Table I] presents the notation used in the entire paper. The paper is further organized in the following section. Section III deals with the related studies on smart cities. Section III covers the existing technologies and their implementation in current and emerging smart cities. Section IV identifies the baseline for emerging to future smart cities requirements. Section $\mathrm{V}$ discusses the open research issues and future directions. Finally, Section $\mathrm{VI}$ presents the conclusion.

\section{RELATED STUDIES}

In this section, the latest state-of-the-art studies are reviewed, and their crucial point of interest are highlighted. Moreover, a taxonomy comparison table has also been represented at the end of the section, which compares the proposed future smart city architecture with current state-of-the-art studies. It can be seen that the reviewed studies lack in several areas of research which majorly includes sixth-generation (6G) networks, WiFi-7, Industry 5.0, Robotic Systems, Human well-being, Heating, ventilation, and air conditioning (HVAC), Pantry Backup, Calamity Backup. These mentioned research topics for future smart cities are critically essential to provide an accessible and sustainable lifestyle; however, the belowreviewed articles lack covering all these critical issues.

HCI saw a significant rise in the recent past after the emergence of the Fourth Industrial Revolution era (4IR). Technological advancements in the field of high-speed computing 


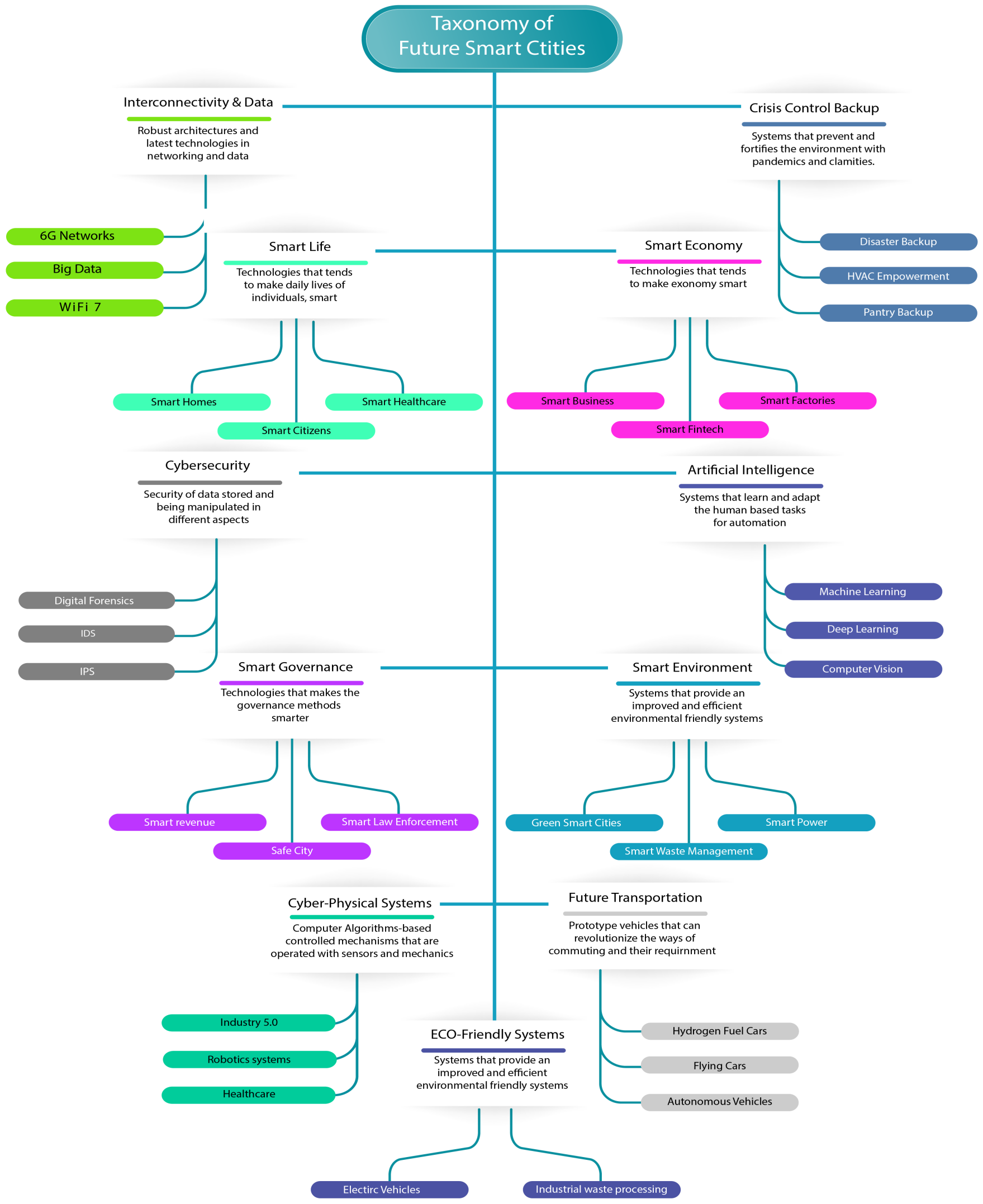

Fig. 2: Taxonomy Diagram of Future Smart Cities 
TABLE I: List of Abbreviations

\begin{tabular}{|c|c|}
\hline Abbreviation & Description \\
\hline $4 \mathrm{G}$ & Fourth Generation \\
\hline 4IR & Fourth Industrial Revolution \\
\hline $5 \mathrm{G}$ & Fifth Generation \\
\hline $6 \mathrm{G}$ & Sixth Generation \\
\hline AGVs & Automated Guided Vehicles \\
\hline B5G & Beyond 5G \\
\hline $\mathrm{B} 2 \mathrm{~B}$ & Business To Business \\
\hline $\mathrm{B} 2 \mathrm{C}$ & Business To Customer \\
\hline $\mathrm{B} 2 \mathrm{G}$ & Business To Government \\
\hline $\mathrm{C} 2 \mathrm{~B}$ & Customer To Business \\
\hline $\mathrm{C} 2 \mathrm{C}$ & Customer To Customer \\
\hline $\mathrm{C} 2 \mathrm{G}$ & Customer To Government \\
\hline CMU-MIMO & Clustering Multi-user Multiple-Input Multiple-Output \\
\hline $\mathrm{CNN}$ & Convolutional Neural Network \\
\hline CPS & Cyber-Physical System \\
\hline CPSS & Cyber-Physical-Social System \\
\hline CSMA/CA & Carrier Sense Multiple Access/Collission Avoidance \\
\hline $\mathrm{CV}$ & Computer Vision \\
\hline DFaaS & Digital Forensics as a Service \\
\hline DL & Deep Learning \\
\hline EHT & Extremely High Throughput \\
\hline eMBB & Enhanced Mobile Broadband \\
\hline EU & European Union \\
\hline G2B & Government To Business \\
\hline G2C & Government To Customer \\
\hline G2G & Government To Government \\
\hline GWSN & Green Wireless Sensor Network \\
\hline $\mathrm{HCI}$ & human computer interaction \\
\hline HCPS & Human-Cyber-Physical Systems \\
\hline $\mathrm{HI}$ & Human intelligence \\
\hline H-IoT & Healthcare Internet Of things \\
\hline HVAC & Heating, ventilation, and air conditioning \\
\hline ICT & Information Communication Technology \\
\hline IDS & Intrusion Detection System \\
\hline IIoT & Industrial Internet of Things \\
\hline IoNT & Internet of Nano Things \\
\hline IoT & Internet Of Things \\
\hline IPS & Intrusion Prevention System \\
\hline IR5.0 & Industrial Revolution 5.0 \\
\hline ISM & Industrial, Scientific and Medical \\
\hline ITU & International Telecommunication Union \\
\hline LEA & Law Enforcement Agencies \\
\hline MELINDA & Multilevel Information Fusion Edge Architecture \\
\hline MIMO & Multiple-Input Multiple Output \\
\hline ML & Machine Learning \\
\hline mMTC & Massive machine type communication \\
\hline mULC & Massive Ultra-reliale Low-latency Communication \\
\hline NFV & Network Function Virtualization \\
\hline OCHA & ordination for Humanitarian Affairs \\
\hline PDS & Public Distribution System \\
\hline RoI & Return on Investment \\
\hline SDN & Software Defined Networks \\
\hline UAVs & Unmanned Aerial Vehicles \\
\hline UL MU-MIMO & UpLink MultiUser Multiple-Input Multiple-Output \\
\hline ULBC & Ultra-Reliable Low-Latency Broad-Band Communication \\
\hline uMBB & Ubiquitous Mobile Broadband \\
\hline uRLLC & low latency communication \\
\hline URLLC & Ultra-Reliable Low-Latency Communication \\
\hline $\mathrm{V} 2 \mathrm{C}$ & Vehicle to Cloud \\
\hline V2D & vehicle-to-devices \\
\hline V2G & vehicle-to-grid \\
\hline $\mathrm{V} 2 \mathrm{H}$ & Vehicle to Home \\
\hline V2I & vehicle to infrastructure \\
\hline $\mathrm{V} 2 \mathrm{~N}$ & vehicle-to-networks \\
\hline $\mathrm{V} 2 \mathrm{P}$ & Vehicle to Pedestrian \\
\hline V2R & vehicle-to-road-side unit \\
\hline V2S & Vehicle to Sensors \\
\hline $\mathrm{V} 2 \mathrm{~V}$ & Vehicle to Vehicle \\
\hline V2X & Vehicle To Everything \\
\hline
\end{tabular}

result in more powerful miniaturized machines available for utilization in every aspect of human life [7]. This phenomenon also significantly shifts a portion of computing activities from cloud computing to edge computing. The linkage of smart cities concerning their various operations and processes in the current literature is comprehensively reviewed in this section.

The authors [8] reviewed advanced healthcare techniques with their technical properties and their different application areas. The authors also deeply reviewed communication technologies with different parametric configurations and then highlights the significance and future aspects of IoT-based health care techniques. The authors in [9] presented a detailed review of smart city foundations and core elements. The smart cities' highest standards are also presented to make a competitive comparison between state-of-the-art smart cities research. It has been discussed in detail how smart cities make living standards comparatively better and wiser across various countries. This research also provides the technical aspects of developing smart cities, yet they present the latest research according to those technical standards.

The authors in [10] proposed energy consumption prediction of electrifying vehicles under a smart city environment. They addressed the necessary causes and their predicted results after implementing the proposed approach based on real-life data with an error rate of $8.44 \%$. The authors in [11] highlighted the issues in the advanced healthcare systems, in their technical and non-technical depths. Hence, they review wearable devices' solutions, including sensors, power management, signal processing, computing architectures, and communication, and highlighted the future research directions. The authors in [12] emphasized maintainable technological systems for future smart cities. The authors also highlighted the life cycle design and recycling procedures of products to examine the adverse effects on human life. Furthermore, the authors also reviewed the computing systems, including their electronic circuitry of essential IoT devices to industry-level data centers. Finally, the authors also highlighted the safety, confidentiality, and effectiveness of maintainable computing for future smart cities.

The authors in [13] deeply reviewed the AI-based developments in the RE region European union (EU). They mainly highlighted energy consumption and efficiency of transformation procedures and consequences of renewable energy by sources such as sun, water, wind, and biomass. The authors in [14] comprehensively study the current recent work done over the region of fog computing concerned with smart cities. The authors also analyzed modern studies' literature reviews and highlighted the future research areas. The authors in [15] highlighted the issues in emerging technologies required to establish future smart cities. Their study includes the IoT, big data, blockchain, AI, data analytics, machine, and cognitive learning, which are implemented for health, energy, transportation, education, public safety to provide a better, easier, and smarter life. The authors in [16] highlighted the role of edge computing and new challenges for the emerging technologies. The authors also critically review the state-of-the-art studies based on edge computing applications in smart cities. The authors in [17] thoroughly surveyed the DL advancements and applications designed for smart cities. The authors also propose the current drawbacks in the model and provide a future research direction in DL. Table II] shows the detailed comparison of the above-reviewed studies. It can be evaluated that the current state-of-the-art studies still lack in providing the latest most technology in all domains of development 
which are necessary for a future smart city of the 21 st century.

The researcher in [18] presents the features critical segments of the information the board structure, surveys different smart city applications, and talks about protection and security challenges related to smart city information. From the point of view of information systems, it is seen that the information utilized in smart city applications is unstructured, coming from heterogeneous sources, i.e., sensors and web-based media, other than others. In this way, the assortment, preparing, examination, the executives, and representation of such information are testing. To play out these undertakings, ongoing advances, i.e., the IoT, sensor organizations, have been utilized.

The researchers in [19] highlight the robustness of ICT that accomplished Smart Cities' idea. In a smart city, a few IoT sensors are sent across a few areas to gather information about traffic, waste, the versatility of residents, and the bits of knowledge acquired from this information are utilized to oversee assets, resources, and so on viably. Profound learning has been utilized widely on the information created by IoT sensors in a smart city by a few specialists. Moreover, an endeavor is made to review a few state-of-the-art DL on Smart City information. A few future exploration headings are recommended toward the finish of the article.

The authors in [20] proposed a systematic literature review (SLR) for the best fog-based methodologies in smart urban communities. Moreover, as indicated by the investigation's substance, a scientific classification is proposed, which falls into three classes: administration-based, asset-based, and application-based. This SLR likewise explores the assessment factors, utilized devices, assessment techniques, benefits, and negative marks of each class. Kinds of proposed calculations in each category are also referenced. Regardless of anything else, by considering different points of view, far-reaching and unmistakable open issues and difficulties are given through grouping future patterns and issues into reasonable sub-classes.

The researchers in article [21], presents an overview is given on a portion of the momentum research work that adds to empowering cyber-physical-social systems (CPSSs). Some significant parts of CPSSs are distinguished, including the improvement from cyber-physical systems (CPSs) to CPSSs, engineering plan, applications, guidelines, genuine contextual analyses, empowering strategies, and organizations for CPSSs. To establish a framework for improving the open savvy world, we propose a virtualization design and a coordinated structure of storing, figuring, and systems administration for CPSSs. Reenactments confirm the presentation improvement of the proposition. Finally, some examination issues with difficulties and potential arrangements are uncovered for scientists in the connected exploration regions.

The authors in [22] present an extensive overview of unmanned aerial vehicle (UAV) networks from a CPS point of view. First and foremost, they survey the nuts and bolts and advances of the three CPS segments in UAV organizations. At that point, they look inside to explore how these parts add to the framework execution by characterizing UAV networks into three orders, i.e., cell level, framework level, and arrangement of framework level. Besides, the coupling impacts among these CPS segments are expressly delineated, edifying to manage the difficulties in every individual angle. New exploration bearings and open issues are talked about toward the finish of this review. With this escalated writing survey, they expect to give a novel understanding of the cutting edge in UAV organizations.

The essential empowering innovations incorporate the correspondence frameworks between the detecting hubs and the processors and prepare calculations for producing a yield from the information gathered by the sensors. Notwithstanding, as of now, these empowering advances are likewise upheld by a few new advances. The utilization of AI has changed the healthcare IoT (H-IoT) frameworks at pretty much every level. The fog/edge worldview is bringing the figuring power near the sent organization and consequently relieving numerous difficulties all the while. In contrast, substantial information permits dealing with a gigantic measure of information. Furthermore, the software-defined networks (SDNs) carry adaptability to the framework while the blockchains track down the most novel use cases in H-IoT frameworks. The IoNT and tactile internet (TI) are driving the advancement in $\mathrm{H}$ IoT applications. The Researchers in [23] digs into how these innovations are changing the $\mathrm{H}$-IoT frameworks and recognize the future course for improving the quality of service (QoS) utilizing these new advancements.

The authors in [24] present an overview of shrewd city activities and dissect their key ideas and specific information on the executive's methods. They played out a solid writing overview and survey by applying an unpredictable writing framework, including terms similar to smart individuals, shrewd economy, keen administration, brilliant versatility, keen climate, and keen living. They additionally talk about various points of view of sensitive coasting urban areas in detail. With the proposed approach, late advances and pragmatic future freedoms for smart urban areas can be uncovered.

Each of these paradigms is further divided into three subsections.

\section{REQUIREMENTS FOR FUtURE SMART CitiES}

Shaping a robust future smart city has several fundamental requirements, which are also regarded as the building blocks of sustainable and advanced technologies. By studying the current state-of-the-art researches in light of the below-mentioned parameters, the requirements for the proposed future smart city is hence justified as below:

\section{A. Interoperability}

The dawn of networking was the future endeavor for the concept of interoperability in today's modern technology. This concept's most significant invention is IoT's supremacy, yet still, under the phase of innovation [25]. The communication of the technologies is a significant aspect of IoT. It allows the devices to be more compatible in the environment. It increases the technology's feature capacity as the capability of interpretation increases the data sharing among the device and lets them work with even more proficiency capabilities. Moreover, User experience is one of the most emphasized parameters in modern technologies. For a comfortable user experience, the interface acts as the foundation stone for that 

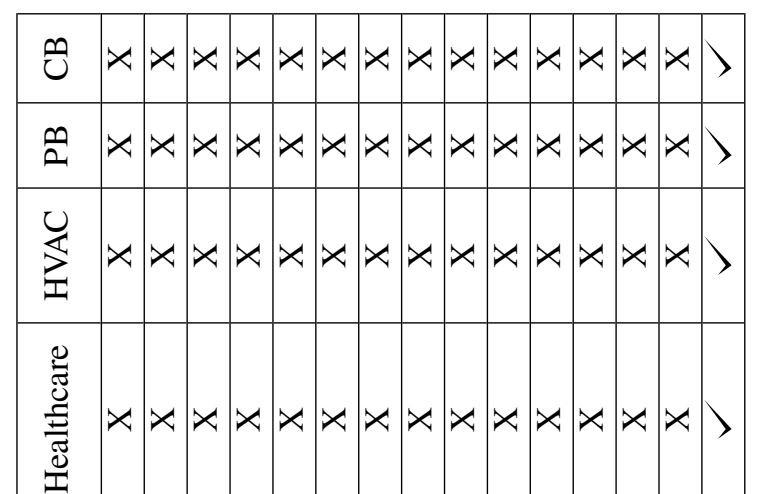

ต $\geqslant|x|>>x \times x \times x|x| x|x|\rangle$

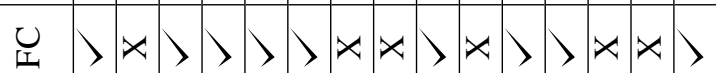
䛼 $x \times x|x| x|x\rangle \times x|x| x|x\rangle$ Z $x \times x \times x \times x \times x|x| x|x\rangle$

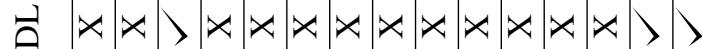
$\left.\sum x|x|>|x|>>x\right\rangle|x| x|>\rangle>$ $\sum|x| x|>| x|>\rangle|x| x|x| x|>\rangle>$ $\stackrel{a}{a}|x\rangle>x|x\rangle|x\rangle>x|x| x\rangle>>$ $\sum x>x|x| x|>| x|x| x|x|>|x\rangle$ $\geq>x|x| x|x| x|x| x|x| x$ $\stackrel{E}{\Leftrightarrow}>x|x| x|x| x|x| x|x| x\rangle$ $\tilde{n}|x\rangle|x| x|x| x|x\rangle|x\rangle|x\rangle>$

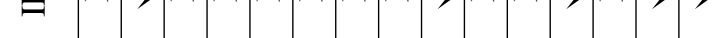

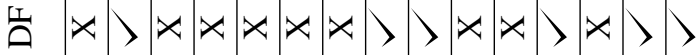
$\approx x \times x|x| x|x\rangle \times x|x| x|x\rangle$

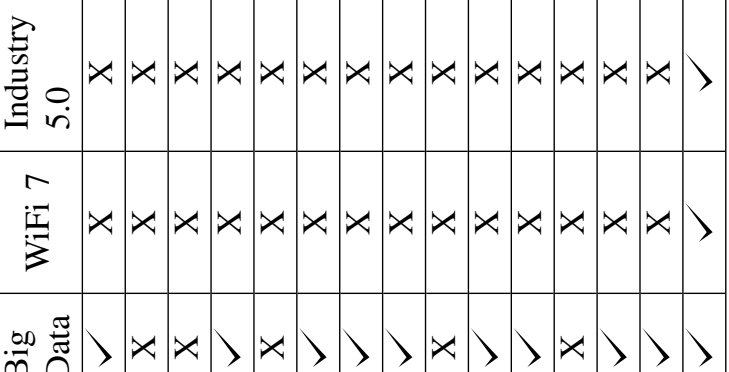
ص丿

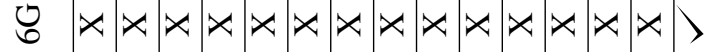

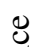
$\stackrel{\circlearrowright}{0}$

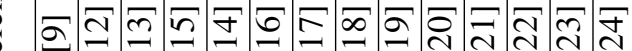
$\frac{2}{2}$ cause. Nevertheless, the interface is also regarded as playing a vital role in incrementing the interoperability in the latest technologies since it allows the users to interact with devices easily yet with significant extensions in features.

\section{B. Scalability}

It is defined as the versatility nature of any product. The scalability of a particular item is also emphasized in modern technologies to ensure suitable performance and efficiency. This parameter also measures the needs fulfilled by a specific technology in consumer requirements [26]. Better scalability also ensures that a product is capable of providing more features to consumers. The more features in the product also save the cost being spent on other items and enhance the usability of the product [27].

\section{Fast Deployment}

The design of the technology plays a crucial role in managing the space and deployment of the technology. In the modern era of technology, sustainable but portable deployment is preferred since it takes less time in implementation with less workforce. The intelligent technologies in construction materials or generic interfaces make the manufacturing procedures efficient and straightforward in terms of cost and time. The authors extensively provide information regarding developing innovative and robust conventional interfaces and deployment procedures for fast deployment.

\section{Robustness}

The rigorous trials in vast research fields are based on outperforming the base studies regarding performance and efficiency. This trend in research tends the upcoming technologies to be more advanced than the previous ones, which has regarded the robustness in the technologies. This robustness is considered to be the innovation as well in modern technology. In the technical terminology of robustness, it can be regarded as the rigorous procedures held at the testing phase of the technology to test the technology's capability to overcome the limitations and errors [28], [29].

\section{E. Eco Friendly and Efficiency}

The fundamental source of power is the electricity that most of the technologies consume. However, the source of power can be based on multiple fuels. With the rise in global warming and adverse changes in global weather conditions [30], research and manufacturing technologies have become highly eco-friendly and efficient oriented. Electronic appliances' power consumption has been significantly decreased with safe environmental emissions [31]. This is necessary for society's global well-being, but it is also a vital parameter in defining the cost-efficient manufacturing and usage of products. 


\section{F. Multi-Modal Access}

IIt is also concerned with the versatility of applications such that having multiple interfaces allows them to outcome the result of input in multiple forms. This technique revolutionizes technology's behavior and usage, especially in mobile computing [32]. The sensors and AI-based algorithms provide a new gateway for developing applications. The extension of multi-modal access in the applications increases the interconnectivity and usability of the technology makes the technology more practical, such as increasing the channels of communication with technology (text, voice, and mail) [33].

\section{Future Smart Cities Application Frameworks}

Smart Cities are based on six fundamental pillars, which are composed of Smart Life, Smart Economy, Smart Governance, Smart Environment, Smart Power, Smart Communication, and Transportation [5]. The use of the latest technology is inevitable for the solution of urban issues in a citizencentric way. This paper groups and distributed smart cities' technological and management attributes to view the allied areas' trends and advancements into seven major categories. These seven categories contain 03 main subcategories, each making the overall 28 taxonomies discussed regarding future smart cities. Up to the authors' knowledge, this paper is the first such comprehensive effort to give such wide coverage in a single paper to shed light on future smart cities by using a top-down approach.

\section{A. Inter-Connectivity and Data}

In an ideal smart city, the citizen of the city benefited by the provision of state-of-the-art, high speed, and smart means of communication as well as transportation [5]. Further to this, connectivity is the backbone of every aspect of a smart city. In a future smart city, the provision of a secure, efficient, high speed, and cross-domain compatible network is the crucial requirement [34]. The transfer of responsibilities among endpoint sensors, edge computing, fog computing, and cloud computing further highlights the necessity of a reliable communication infrastructure providing real-time data to the participating nodes. This transfer reduces the amount of data communicated to the cloud as the raw data is now being processed at edge level using fog computing and then processed information is then forwarded to cloud servers for analysis [35].

Huge bandwidth requirements in the era of a smart city that once seemed impossible to achieve are now made possible by the $5 \mathrm{G}$ networks. $5 \mathrm{G}$ is the there reference term used for the fifth generation of high-speed communication technologies started back in 1980 for the sole purpose of tackling mobility in design [36]. The high speed promised by $5 \mathrm{~g}$ is already anticipated to replace the home WiFi networks as they offer a mere speed compared to the one available in 5g's proper implementation. Being planned in a higher spectrum, the speed promises of beyond 5G technologies are enough to fulfill today's and upcoming technologies' bandwidth requirements. It will also generate around USD 13.2 trillion by 2035 , as anticipated by World Economic Forum's white paper on 5G technologies.
Focusing on enhanced mobile broadband (eMBB) provision, ultra-reliable low latency communication (uRLLC), security, massive machine type communication (mMTC), and power efficiency are the key drivers behind the wide adoption of $5 \mathrm{G}$ in current communication networks. The challenge of fast, efficient, and rapid data processing is tackled by the European telecommunications standards institute (ETSI) by introducing multi-access edge computing (MAEC). It processes the data at the edge level to forward processed/required information onward to decrease the communication load [37].

In today's cloud-centric smart city network, the major challenge is providing a secure, reliable, efficient, and fast network backbone. In this regard, measures were taken through research to minimize traffic flow volume from host to cloud using edge and fog computing. This way, the cloud's huge computing and storage resources can also be efficiently utilized [35].

For future smart cities, the network complexity is anticipated to grow many folds as IoT's incorporation makes devices able to talk with the devices and other nodes. This ocean of nodes uses complex architecture composed of the traditional network means, proprietary protocols, heterogeneous means of communication, infrastructure-based, ad-hoc, and mobile formations based on wired and wireless electrical and optical technologies. High speed and data rates offered by $5 \mathrm{~g}$ and upcoming tera-hertz based $6 \mathrm{~g}$ technologies will cater to the need and requirements of communication of this ocean of devices. Figure 3 presents how high speed communication connecting smart cities.

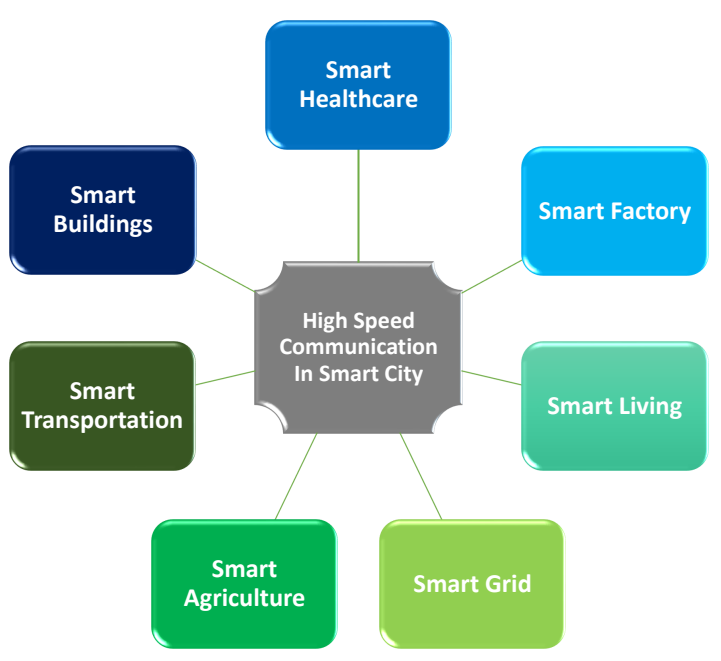

Fig. 3: High Speed Communication Connecting Smart Cities

Besides the technological and economic promises made by the $5 \mathrm{~g}$ and beyond communication technologies, there are specific challenges to consider before reaping the benefits mentioned above. The first among all is the design level efficiency, which is regarded as the strength and base for improvements gained and results in incompatibility issues with the existing infrastructure of $4 \mathrm{G}$ and below. Currently deployed $5 \mathrm{G}$ testbeds are built along with the existing infrastructure, but they cannot unleash the true potential of $5 \mathrm{G}$ based on a higher 
spectrum. The requirement is to design the network with a core built for 5G specification, including the two main components, network function virtualization (NFV) and software-defined networks (SDN). Another challenge is translating the need for the IoT in connection profiles, bandwidth requirements, and coverage scopes.

Connection and provision of network services to mobile phones are entirely different and less complex, as mobile service provisioning is similar in terms of usage pattern. On the other hand, for IoT, every kind of device group have their requirements. Then based on the deployment scenarios, the same group of IoT devices may possess veracity. Connecting a mobile phone, connecting a car, connecting a fire detection system, and connecting a water meter to the internet have their specifications and requirements. Some of them may require lower bandwidth, whereas some required higher, some need a shorter burst of data to be transferred, whereas some may have to send large amounts, some devices have to communicate over a shorter distance. Whereas some may be required to communicate at higher distances, some devices may have less frequency of data connection. In contrast, some may have more frequency of connectivity with network infrastructure and so on [38]. International telecommunication union (ITU) is aggressively working on the standardization of ML usage in the network design and development based on 5G technologies [39].

For future smart cities, the importance of a high-speed communication backbone that is secure, resilient to attack, always available, and has the required capacity and bandwidth to fulfill the growing needs is the basic building block. It is crucial to take care of all those challenges to these future networks and evaluate them regarding requirements arising from technological advancement in smart cities. It becomes the future research area to identify the high-speed network requirements in future smart cities where network advancement evaluation can be done regarding their deployment in future smart city scenarios to identify gaps and limitations and their solutions and remediation.

Another challenge is to effectively use ML and DL technologies to analyze the node behavior to effectively suggest the workaround for the future and help intelligently heal the network infrastructure to disrupt services in some portion of the network. Usage of edge computing and its impact on upcoming next-generation networks and computing were surveyed comprehensively in [40]. These edge computing technologies are now becoming game-changing both for the following generation networks and computing requirements to continue the era of IoT.

1) $6 G$ Networks: $5 \mathrm{G}$ communication was evolved at a commercial scale in the year 2020. Many developed and underdeveloped countries now have a $5 \mathrm{G}$ commercial network available for everyday customers. The research community is already looking into the beyond $5 \mathrm{G}$ (B5G) or, in more concise words, $6 \mathrm{G}$ based network, which is targeted to fulfill the need of communication requirements in 2030 [41].

Though the $5 \mathrm{G}$ offers the solution for bandwidth and connectivity requirements of current and future applications, the trend emerging due to such high-speed network offerings is exponential growth. Not only the high bandwidth but low latency and reliability of the network are also very significant [42]. The smart city architecture is also converging to chop the bandwidth by offering technological advancements and control to focus on more areas of the smart city previously out of the scope. This will leads to the need to have more advancements in the current $5 \mathrm{G}$ offerings. Rich multimedia based application, city-wide monitoring solutions with live audio-video, enhancements in screen resolution (like FHD, $4 \mathrm{~K}$, etc.), communication between the device to device/device to cloud/device to customers/cloud to the customer, and increasing subscriber base of mobile communication will results in estimated 5+ Zeta-Bytes per month till 2030 [43]. To accommodate this much bandwidth, research is underway to develop a new set of standards and technologies to become the sixth generation communication infrastructure. The use cases of next-generation communication technology like high fidelity communication involving holographic and smart cities level high-speed communication were discussed w.r.t. their fulfillment by $6 \mathrm{G}$, current challenges to $5 \mathrm{G}$, and their solution promised by $6 \mathrm{G}$ are clearly explained in [44]. Figure 4 presents the transformation of $5 \mathrm{G}$ to $6 \mathrm{G}$.

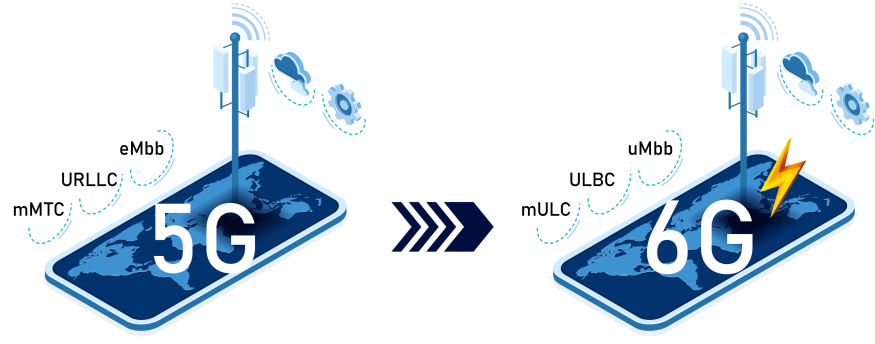

Fig. 4: 5G To 6G Transformation

Major research activities in this high-speed mobile connectivity include eMBB, primarily used for customer-oriented high-speed mobile internet access for multimedia and rich media applications. URLLC in the mission-critical application environment where the requirements are strict for a reliable and consistent low latency network to provide reliable connectivity. The mMTC focuses on the provision of connectivity to a large number of low-cost, low-power devices to communicate while consuming as much less power as possible [45]. The upcoming improvements in this area will result in ubiquitous mobile broadband ( $\mathrm{uMBB}$ ) providing coverage to the whole planet in the $6 \mathrm{G}$ network, eventually upgrading eMBB. This uMBB will cater to bandwidth enhancements expected to cover minimum $1 \mathrm{Gbps}$ per user to manage services like mobile virtual reality. Another critical improvement will be an ultrareliable low-latency broadband communication (ULBC) to provide low-latency, highly reliable network connectivity with higher data throughput for automated guided vehicles (AGVs), robotics, and UAVs. To deploy massive sensors and actuators in verticals, massive ultra-reliable low-latency communication (mULC) will upgrade mMTC by utilizing and combining features from mMTC and URLLC. Seven critical use cases of URLLC as defined in [46] will be further improved and implemented in $6 \mathrm{G}$ and beyond. 
Key improvement indicators for $6 \mathrm{G}$ concerning $5 \mathrm{G}$ are as under [41].

- Peak Data Rate from 20Gbps in 5G to $1 \mathrm{Tbps}$ in $6 \mathrm{G}$

- User-Experience Data Rate from $100 \mathrm{Mbps}$ in 5G to $1 \mathrm{Gbps}$ in $6 \mathrm{G}$

- Latency from $1-4 \mathrm{~ms}$ in $5 \mathrm{G}$ to $10-100 \mathrm{Us}$ in $6 \mathrm{G}$.

- Mobility up to $500 \mathrm{Km} / \mathrm{h}$ in $5 \mathrm{~g}$ to up to $1000 \mathrm{Km} / \mathrm{h}$ in $6 \mathrm{G}$

- Connection Density from $106 / \mathrm{Km} 2$ in $5 \mathrm{G}$ to $107 / \mathrm{Km} 2$ in $6 \mathrm{G}$

- Network energy efficiency in 6G 10 to 100 times better than $5 \mathrm{G}$

- Peak spectral efficiency from $30 \mathrm{bps} / \mathrm{Hz}$ in $5 \mathrm{G}$ to 90 $\mathrm{bps} / \mathrm{Hz}$ in $6 \mathrm{G}$

- Area traffic capacity from $10 \mathrm{Mbps} / \mathrm{m} 2$ in $5 \mathrm{G}$ to 1 Gbps/m 2 in $6 \mathrm{G}$

- Reliability from $99.999 \%$ in $5 \mathrm{G}$ to $99.99999 \%$ in $6 \mathrm{G}$

- Signal bandwidth from $100 \mathrm{MHz}$ in $5 \mathrm{G}$ to $1 \mathrm{GHz}$ in $6 \mathrm{G}$

- Positioning accuracy from $10 \mathrm{~m}$ in $5 \mathrm{G}$ to $10-100 \mathrm{~cm}$ in $6 \mathrm{G}$

Figure 5 in [47] and referenced section in the paper comprehensively covers the mobile network security landscape as well as highlighting the key areas of $5 \mathrm{~g}$ security while focusing on issues related to critical technologies, physical security, and privacy to conclude with the emphasis on security and standardization.

Critical Analysis: Though current communication technologies efficiently manage the current communication/bandwidth requirements and need more smoothly, the anticipated growth in the connected system/devices with the internet and higher bandwidth requirements will make the currently available resources scarce very soon. Advancements into the smart cities' architecture and inclusion of further aspects of city management into the umbrella of SMART will eventually result in more connected arenas and more bandwidth and connectivity requirements. A big chunk of bandwidth can also be used by the increased monitoring and control systems deployed for law enforcement, disaster management, and smart home purposes using audio-visual technologies. 6G will be designed to fulfill the bandwidth requirements keeping in view the increase anticipated till 2030.

2) Big Data: The IoT is all about significant data generation. Its usage and adoption of IoT-based devices and sensors in the automation of city infrastructure will eventually generate many big data islands in various aspects of a smart city. In this regard, a framework was proposed by [48] which targets sustainable smart cities able to illustrate the informational landscape of smart city build up on the cores of big data and IoT and ultimately brings the enhancements in the sustainable physical landscape. Different aspects of data must be analyzed carefully concerning future smart cities' requirements, as summarized below.

Data Volume: The overall system of the smart city already fell into the big data problem, and continued conversion of traditional functionalities of the city into smarter ones added more and more data to generate, refine, and process. This results in the challenge of data storage, communication, and processing capabilities to cope up with the pace of increase in volumes of data [49]. The costs of managing the everincreasing data volumes are enormous, and they must be planned and addressed properly during the feasibility study of the smart city incorporation. Figure 5 presents the management of the big data.

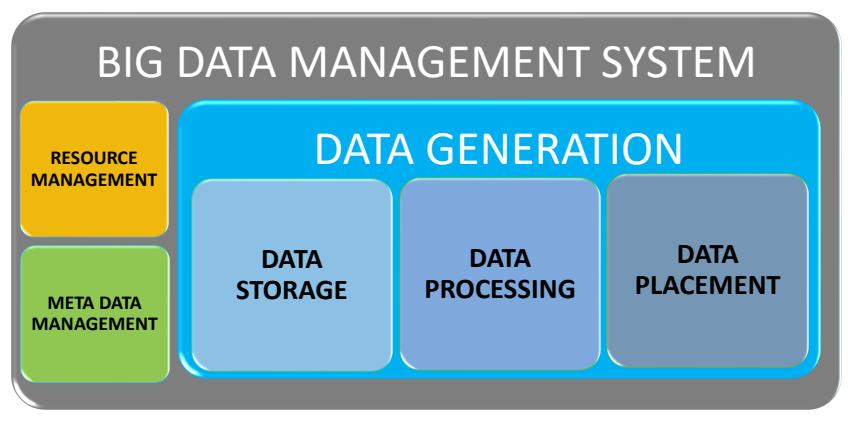

Fig. 5: Big Data Management

Data Redundancy To get the system's insight more accurately, one way usually utilized is to increase the number of sensors used to monitor the states. This results in redundancy as more sensors now report the same state with a minor variation based on sensors' placement. The purpose behind big data analytic is to identify the patterns hidden behind the data and results informing the actual representation of the data [50]. The more data volume we have, the more storage, communication, and processing overhead we will face with the benefits of a rise in inaccuracy. To resolve such overheads, various techniques are applied [51]. These techniques may include (but are not limited to) feature reduction, feature selection, time scale management, statistical means, and other techniques.

Data Cleaning The data at the node level may need cleaning before its final submission to the system. The cleaning may be done on outbound, missing, or incorrect values as the volume of data at this level make it impossible to manage the cleaning process humanely. Hence the ML-based techniques are used for this purpose. Having the right tool for the said results in increasing the pace with which data can be fetched, imported, processed, cleaned, filtered, stored, and exported [52]. Figure 6 presents the challenges faced by big data.

Data Discritization Due to some needs and requirements, data must sometimes be changed from quantitative to qualitative or vice versa. Though data discretization requirements can arrive in any research most of the time, it occurs while handling medical/healthcare-related data [53]. Special care is required to define the categories and scaling of the values being converted. The approaches and schemes must be standardized on the system level to balance the converted values for their effective utilization at the system scale.

Data Velocity The data velocity is the rate of generation of data and can be measured at the component level or the system level. The velocity of the data in the smart city is increasing exponentially. There are two prime reasons behind this phenomenon. The first is the conversion of aspects of 


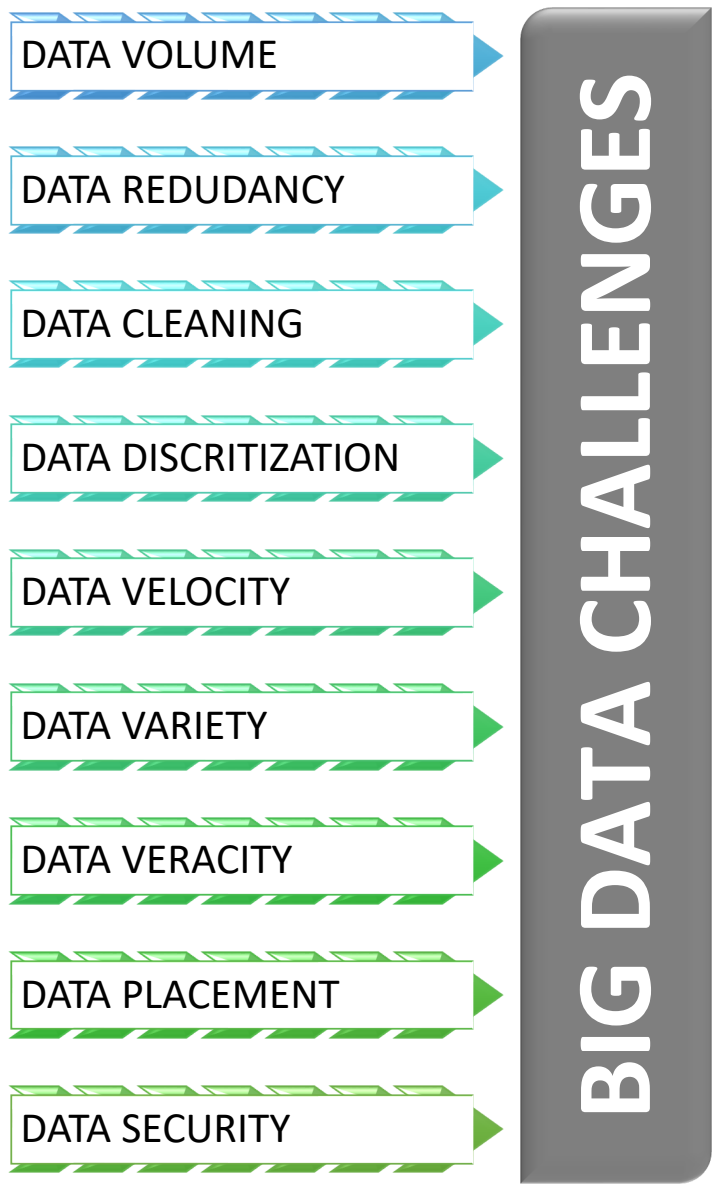

Fig. 6: Big Data Challenges

a smart city from traditional means to technology-based solutions. The second is the technological advancements that bring the state monitoring's precision by adding more and more sensors, especially after the IoNT. The more nodes the system has, the more data it needs to manage. The timely communication of this real-time system's data with proper security measures is a prime task in every smart city aspect.

Data Variety Due to the absence of standardization in IoT, mostly the sensors used proprietary formats. Hence, the system has to handle output in various formats generated by the devices provided by the different vendors. This challenge is faced more commonly in applications related to environment [54]. Extracting and merging information from such vendor inter-incompatible formats and standardization and availability to the whole system is another challenge. Further complexity can be added to this while managing the unstructured data.

Data Veracity The challenge of converting uncertainty on the data to certainty by performing different checks and filters is to solve before processing the system's upper hierarchies. The data may contain biases, noise, or abnormal readings, and such things should be normalized before the data is used for decision making. It is very pertinent to measure if the data is a relevant and suitable candidate for further analysis or not [55].
The high volume of data further limits the time availability of measuring veracity.

Data Placement Due to the exponential increase in data generation and storage and processing capabilities, the technology is shifted from data-centric to knowledge-centric. The requirements arise for the availability of efficient, fast, and scalable support to the big data management [56]. The data may have different values within the system's components, and according to it, the data may be placed and process at different levels. The most common levels are at the device level, edge level, fog level, and cloud level. Edge and fog computing result in prime savings in computing and communication resources at the cloud level.

Data Security Data is the most crucial asset in any system. Maintaining its confidentiality and integrity is a sensitive task for every system designer. Furthermore, the system and its subscribers depend on its in-time availability that is also a challenge. The data in any system typically has two states. i.e., data in transit and data at rest. The data's confidentiality can be achieved using various ways on disks, networks, and processing channels. Wireless technology increasing usage due to ease of networking provided by it also possesses privacy and security concerns that need to be addressed [57].

Critical Analysis: Data is becoming the new gold in the upcoming technology-oriented world where everything is based on the careful collection and analysis of data. Data dependency brings new challenges in collecting, handling, transporting, storing, analyzing, processing, and discarding data regarding confidentiality, integrity, and availability. Big data analysis and ML are becoming more involved in everyday life aspects, and AI involvement in automation to the existing processes and tasks [58]. However, to fully reap big data benefits, the challenges related to big data must be addressed. In the long run, these challenges usually increased exponentially; hence an initial vision, long-term planning, strategy development, goals achievement process, and then procedures and actions to achieve those goals are required for successful big data analytic in the given scenario.

3) WiFi 7: WiFi is the technology used in consumer wireless networks for more than 15 years. The wide adoption and popularity of $\mathrm{WiFi}$, which is technically known as IEEE's 802.11 families of protocols, is based on a license-free spectrum of $2.5 \mathrm{GHz}$ and $5 \mathrm{GHz}$. This unlicensed spectrum is also known as the industrial, scientific, and medical (ISM) band. Carrier sense multiple access/collision avoidance (CSMA/CA) is used as a MAC layer protocol for WiFi communication, making it half-duplex [59]. Extensive use of WiFi in consumers and enterprise networks and public points like airports, hotels, and restaurants raises the need for advancements in connectivity, bandwidth, and speed. The adoption of WiFi is now a prevalent thing and is an essential component of smart homes, many of whose components rely on the WiFi technology for device and device to consumer communication [60]. Figure 7 presents the comparative analysis of the bandwidth increase between $\mathrm{WiFi}$ generations.

802.11ax, commonly known as WiFi-6, is head to head in implementation along with $5 \mathrm{G}$ communication networks. $802.11 \mathrm{n}$ and $802.11 \mathrm{ac}$, commonly termed as WiFi-4 and WiFi- 


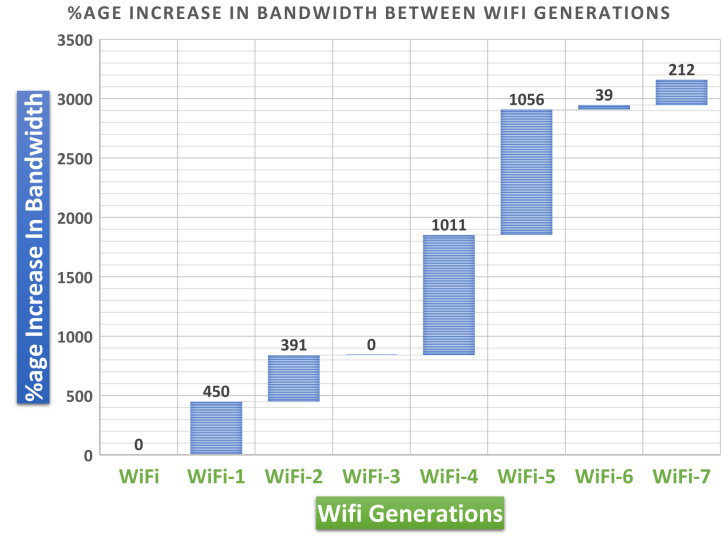

Fig. 7: Comparative Bandwidth Increase Between WiFi Generations

5 , are already implemented in current consumer-level products, and WiFi-6 is already heading its way to the consumer level. Multiple-input multiple-output (MIMO) was the base reason for having high bandwidth possible in WiFi-4 and WiFi-5. 802.11 be extremely high throughput (EHT) will be expected to be designated as WiFi-7. It is still under intense research, and the initial draft for the protocol is expected to be released in March-April 2020 with expected technology roll-out to the consumer market in 2024 [61].

WiFi6 and beyond are converging MIMO into upLink multiuser multiple-input multiple-output(UL MU-MIMO). WiFi6 supports MU-MIMO technology providing 8 data streams at a time. WiFi7 extends it to 16 data streams by utilizing clustering multi-user multiple-input multiple-output (CMUMIMO) technology. WiFi 6 maximum supported throughput of 9.6Gbps, and WiFi 7 design improvement will expand to 30Gbps, more than three times. WiFi7 also uses the 4096QAM signal modulation method instead of the 1024-QAM signal modulation method used by WiFi6. The significant shining improvement brings into WiFi7 is the introduction of a $6 \mathrm{GHz}$ band. Previously 802.11 only operates in 2.4 and $5 \mathrm{GHz}$ bands, but for the first time, the $6 \mathrm{GHz}$ band will be utilized in WiFi7. Another aspect under research is the effective utilization of the available spectrum, and DL is also being utilized for solving some of these challenges, especially on the MAC layer of wireless network [62].

Critical Analysis: Keeping in view the implementation of IoT at the consumer level and the edge and fog computing and their interactive connectivity with cloud-based systems, WiFi7 will future proof the home/enterprise LAN networks in terms of bandwidth and connectivity requirements. In WiFi7, higher frequency utilization for communication will also resolve the challenges limiting the advancements due to limitations in the $2.4 \mathrm{GHz}$ and $5 \mathrm{GHz}$ band and allow the author to achieve the targets without getting bounded by the previous limitations. Table III presents interconnective and data literature.

\section{B. Cyber Physical Systems}

Automation in factories is a traditional concept and was widely implemented to save time and resources. However, this approach further moves in a smarter aspect to impart the technological advancements into the traditional automation concept in the current era. This smartness revolves around bringing intelligence to automated systems. Hence the existing digital factories are now becoming intelligent factories [63]. Figure 8 presents the cyber-physical security approach.

The next generation intelligent factories are built on the layered model having an End-user/management terminal layer, a cloud-based data analysis and decision support layer, a network layer looking after all levels of communications, and a physical resource layer managing the actual work done on the production line. Each layer then manages a couple of the devices and controller to perform the desired action [63]. These advanced technology-based smart factories are known as a CPS. The security of CPS is a hot research topic nowadays. The above referred layers of the CPS have the standard of the component at each layer under computer, communication, storage, and physical world interaction using sensors and actuators of various kinds and nature. The security in CPS mainly revolves around the privacy issues, dependability of components and layers, resiliency against accidents and attacks, interaction among layers and components and their coordination, operational security, system hardening, and most of the time, system availability [64], [65].

1) Industry 5.0: In today's technology-oriented world, the shift is seen from comparatively less developed countries excelling in the latest technologies faster than their counterparts. Being at the top of the technological horizon is becoming more difficult nowadays [66]. The approximately 250 yearlong history of industrial revolutions shows a gradual shift in the revolutions' $\mathrm{x}$-centric behavior from machine and technology from the first three generations to cognitive intelligence and machine to machine intelligent communication in the fourth industrial revolution. The same is now going to become human-centric in Industry 5.0. It will make humans only in the design and control position. The laborious work will become fully automated and smart controlled by intelligent machines looking after every aspect of the production process. It will result in everything customized to work for customercentric industries. Each product from those industries will be customized as per the customer's requirements and personality, which will result in more convenience and satisfaction of the customer, lesser returns, and more value for money being made to order.

AI will be heavily used in industrial revolution 5.0 (IR5.0) to change management, transformation management, customercentric production, and research and development. This era's major governing components will be IoT, big data, ML, AI, CPS, and direct human-robot interactions [67]. Another novel concept introduced by [68] is of human-cyber-physical systems (HCPS), which combines human intelligence (HI) with trained AI solutions to bring the cognitive smart CPSs. Cognitive intelligence is build using sensors (for taking the input from the environment and targets). It uses a translator 
TABLE III: Summary of Interconnective and Data Literature

\begin{tabular}{|c|c|c|}
\hline Ref. & Proposed Work & Outcome \\
\hline $\mid 5]$ & $\begin{array}{l}\text { Sheds light on different aspects of a smart city, } \\
\text { including power, energy, water health, and trans- } \\
\text { portation. }\end{array}$ & $\begin{array}{l}\text { After introducing the smart city in current technological developments, data } \\
\text { analytics for solar energy, blockchain application in microgrid infrastructure, } \\
\text { smart water management using Zigbee-based network, and smart temperature } \\
\text { sensor deployment are discussed in detail. }\end{array}$ \\
\hline$[\overline{34}$ & $\begin{array}{l}\text { Discussed security challenges in smart farming and } \\
\text { ways to tackled them. }\end{array}$ & $\begin{array}{l}\text { Briefly discusses the potential cyber attack on the smart farming ecosystem } \\
\text { and outlines a multi-layered security architecture. }\end{array}$ \\
\hline |36 & $\begin{array}{l}\text { Implementation and benefits of } 5 \mathrm{~g} \text { concerning the } \\
\text { fourth industrial revolution were discussed in this } \\
\text { article. }\end{array}$ & $\begin{array}{l}\text { The prominent feature of this article is its use-case-driven approach towards } \\
\text { next-generation networks. Factors to improve the network coverage and quality } \\
\text { to provide the best service to the consumers were discussed. }\end{array}$ \\
\hline$|\overline{35}|$ & $\begin{array}{l}\text { Work on a solution that can be deployed on edge } \\
\text { devices to provide a reliable and secure communica- } \\
\text { tion channel between end-user IoT devices and fog } \\
\text { computing. }\end{array}$ & $\begin{array}{l}\text { This paper proposed the composition of the complex services-based framework } \\
\text { using fuzzification and employing reinforcement learning techniques with } \\
\text { inbuilt intrusion detection to provide an efficient communication solution. }\end{array}$ \\
\hline$|38|$ & $\begin{array}{l}\text { Discussed the open and upcoming challenges to } \\
\text { advanced telecommunication networks like } 5 \mathrm{~g} \text { and } \\
\text { tackled them efficiently. }\end{array}$ & $\begin{array}{l}\text { Discussed challenges and possible solutions in the telco domain in the area } \\
\text { under discussion in agile virtual networks, massive IoT-based customer-level } \\
\text { connected objects, data-driven networks, and cybersecurity. }\end{array}$ \\
\hline$[41]$ & $\begin{array}{l}\text { Look into the factor enabling the grounds for } 6 \mathrm{G} \\
\text { networks to be deployed till } 2030 \text {. }\end{array}$ & $\begin{array}{l}\text { This paper discusses technical rules and regulations, gains, benefits, and tech- } \\
\text { nological improvements for the next generation of high-speed communication } \\
\text { networks. }\end{array}$ \\
\hline [45] & $\begin{array}{l}\text { Consumers and industries will add unique bandwidth } \\
\text { requirements in upcoming years, exceeding the cur- } \\
\text { rent offering of high-speed } 5 \mathrm{~g} \text { networks. This paper } \\
\text { focuses on the solutions to technology advancements. }\end{array}$ & $\begin{array}{l}\text { radio access networks (RAN) and their architecture and how to improve the } \\
\text { architecture to enhance the quality and services of current RAN networks } \\
\text { is the main target of discussion in this paper. Multi-access edge computing, } \\
\text { virtualization of network function (NFV), software-defined networks (SDN), } \\
\text { multimeter wave, MIMO, massive machine type communication are among } \\
\text { the topics reviewed. }\end{array}$ \\
\hline$[\overline{48}]$ & $\begin{array}{l}\text { IoT becomes the most critical factor for various } \\
\text { monitoring and control-related tasks in sustainable } \\
\text { smart cities, and applications based on them can play } \\
\text { key roles in such deployments. }\end{array}$ & $\begin{array}{l}\text { Provides state the in-depth art coverage in the form of a framework that } \\
\text { elaborates a conceptual framework to build a useful model of sustainable smart } \\
\text { cities. }\end{array}$ \\
\hline$[\overline{49}]$ & $\begin{array}{l}\text { Discuss big data concerning the organization's needs } \\
\text { and requirements with special consideration on is- } \\
\text { sues faced during big data handling. }\end{array}$ & $\begin{array}{l}\text { Big data variety has the most negative impact on the organization's milking } \\
\text { benefits from the big data. The study discusses opportunities and circumstances } \\
\text { that can harvest the most benefits out of big data analysis. }\end{array}$ \\
\hline $\mid \overline{60}$ & $\begin{array}{l}\text { Provide comprehensive coverage of WiFi current } \\
\text { technologies and their impact on the industry and } \\
\text { consumers. }\end{array}$ & $\begin{array}{l}\text { The study outlines the trends, technologies, and in-depth details of what WiFi } \\
\text { current enhancements are and their pros and cons. The technical details are } \\
\text { well covered, with enhances insight into them. }\end{array}$ \\
\hline $\mid \overline{61}$ & $\begin{array}{l}\text { Provide comprehensive coverage of IEEE's } 802.11 \\
\text { current and upcoming advancements and their im- } \\
\text { pacts. }\end{array}$ & $\begin{array}{l}\text { This article provides coverage and analysis along with technological enhance- } \\
\text { ment in WiFi, focusing on revision } 802.11 \text { be, which will be the WiFi } 7 \text {. }\end{array}$ \\
\hline
\end{tabular}

to translate the input data into information, modeling for numerical analysis, semi-supervised learning of the system for making it smart for future decision making and modeling of intelligent decision making for future unsupervised actions, analysis and upload of the data to cloud and human-system interaction for running the whole system [69]-[71]. Figure 9 presents the concept of smart factories in todays' world.

The overlapping interaction between the business, customer, and technology is the base of the industry 5.0 innovation [72]. These, too, are heavily involved in the smart cities' architecture at their level. Their interaction will also impact their utilization within the smart cities system and eventually bring more citizen-centric citizen-centric smart cities. The innovations in these domains will eventually end in the overlapping intelligently communicating system, which was previously based on the interaction among each other [73]. The 5.0 is now becoming a symbol various areas and used as a symbol to the customer/citizen-centric approach [74] such as Retail 5.0 (a customer-centric next-generation business framework outlines) [75], Government 5.0 (A citizen-centric government framework) [76], concepts of Entertainment 5.0 and Education 5.0 as discussed in [74], overview of Production 5.0 without mentioning the term (highly customized production approach towards customers variable requirements) in [77], Industry 5.0 with discussion on impacts on customization trend on new market opportunities and environmental impacts in [78] are to name a few. Figure 10 presents the industrial revolutions chronology of smart cities.

\section{Critical Analysis:}

Mass customization concerning individual customer demand is the next big thing to become a competitive advantage in the coming days. The Industrial Revolutions 5.0 revolves around the customer and will be more beneficial for the customer. The customer will get the products customized to the needs, requirements, and new dimensions of customer personality, mood, and preference. The same paradigm will change the future smart city into becoming citizen-centric by offering and modifying the smart city services to citizens as per the need, demands, and living context by integrating cognitive intelligence into the already automated processes.

2) Robotics Systems: Robotics has their history back when humans start mechanics to bring ease to their work. However, modern robotics counts as the 1 st generation of proper robotics since electronically managed machines perform specific tasks independent of continuous human supervision. Since then, the concept of automation gradually evolved into intelligent automated machines capable of making decisions based on the situation before taking actions and performing the task as per the environment requirements.

Automation technologies are continuously used throughout 


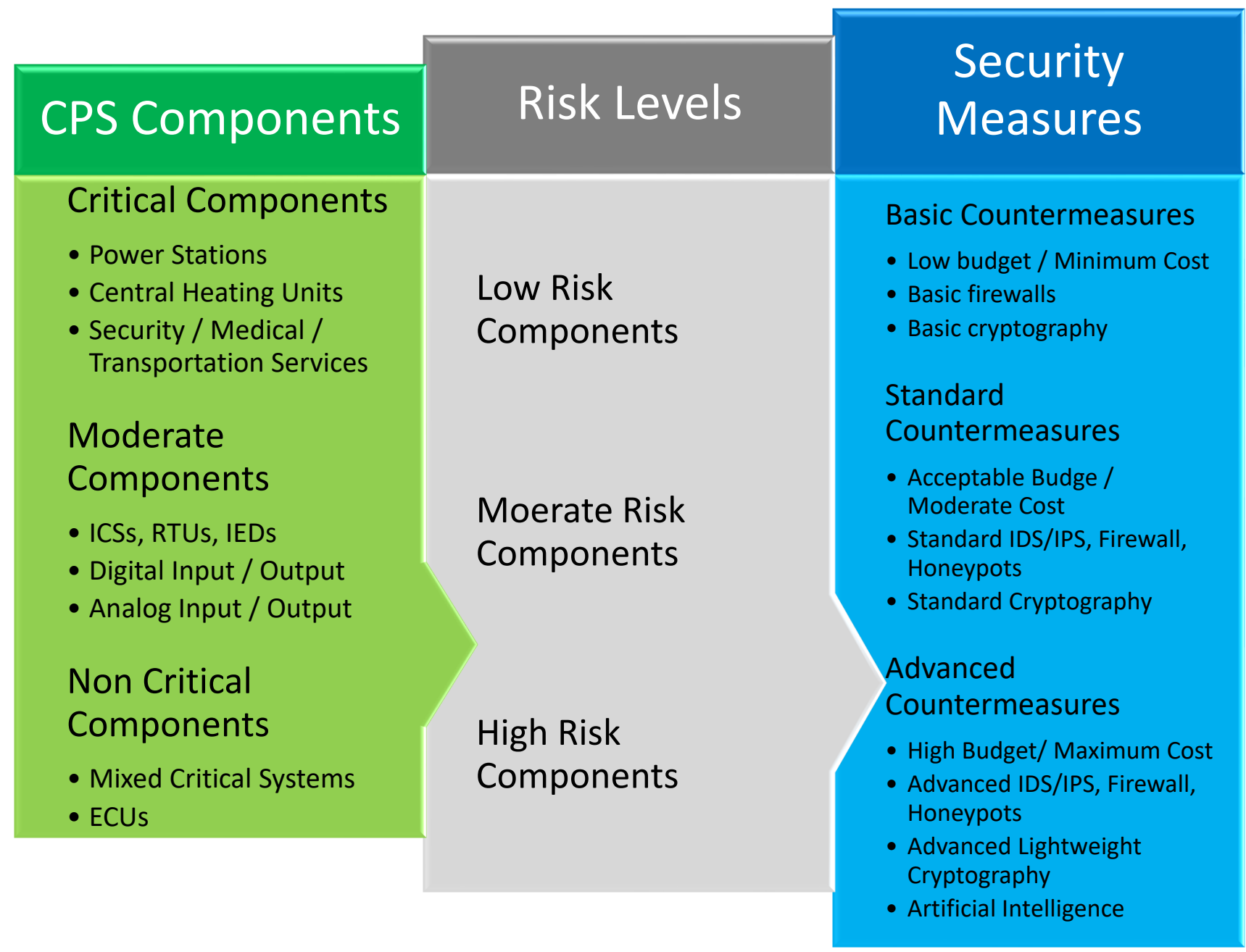

Fig. 8: Cyber Physical Security Approach

the world in smart cities. Robotics is a crucial part of this trend. Both of them are now become the natural partner in the cyber smart city arena [79]. For technological advancements, smart cities are used as testbeds to implement automation of various processes, services, and facilities using robotics and AI. AI and robotics can be used in various aspects of smart cities, including automation (i.e., big data, ML, DL), decision making ( i.e., economy, governance), education (one-stopshop, robotics assistants), smart infrastructures (i.e., sensors, robotics monitoring) and smart mobility (i.e., autonomous transportation, robot garages) [80]. Figure 11 presents the generations of robotics in smart cities environment.

A proposal was made in [81] about using a 3-tier approach in the city's crisis control and management in which control of crowd was discussed using AI and robotics. The system gets the data from the camera sensor. It analyzes it to interpret the situation's intention and intensity by processing the cloud using AI and deciding the necessary actions to implement crowd control, which got implemented using connected robots.

In the same way, robotics can be used for handling emergency solutions, where the use of robots and drones can effectively support crisis management. Drones usage in fire fighting tasks is also proven helpful against monitoring, prevention, surveillance, and extinguishing [82]. Russia is going to use advanced robotics in its emergency operations like bringing medicine immediately to the affected area, shifting of the wounded person from the site, provision of tools and equipment in disaster situations, etc. [83]. A training data set for automated surgical operations by robots and methods for its propagation and sharing to other robotic platforms were discussed in [84] in order to develop the capability to perform emergency medical/surgical procedures using AI to tackle the highly emergent situations where availability of healthcare professionals risks lives. Those surgical maneuvers were compiled as a library for sharing for continuous learning and improvements. The latest advancements and research aspects were explored in $[85]-[90]$ as published in the first month of 2021, showing the active research on autonomous robotics in various fields of public life.

\section{Critical Analysis:}

AI-backed autonomous robotic systems are being researched to assist human counterparts in various fields of human life. 

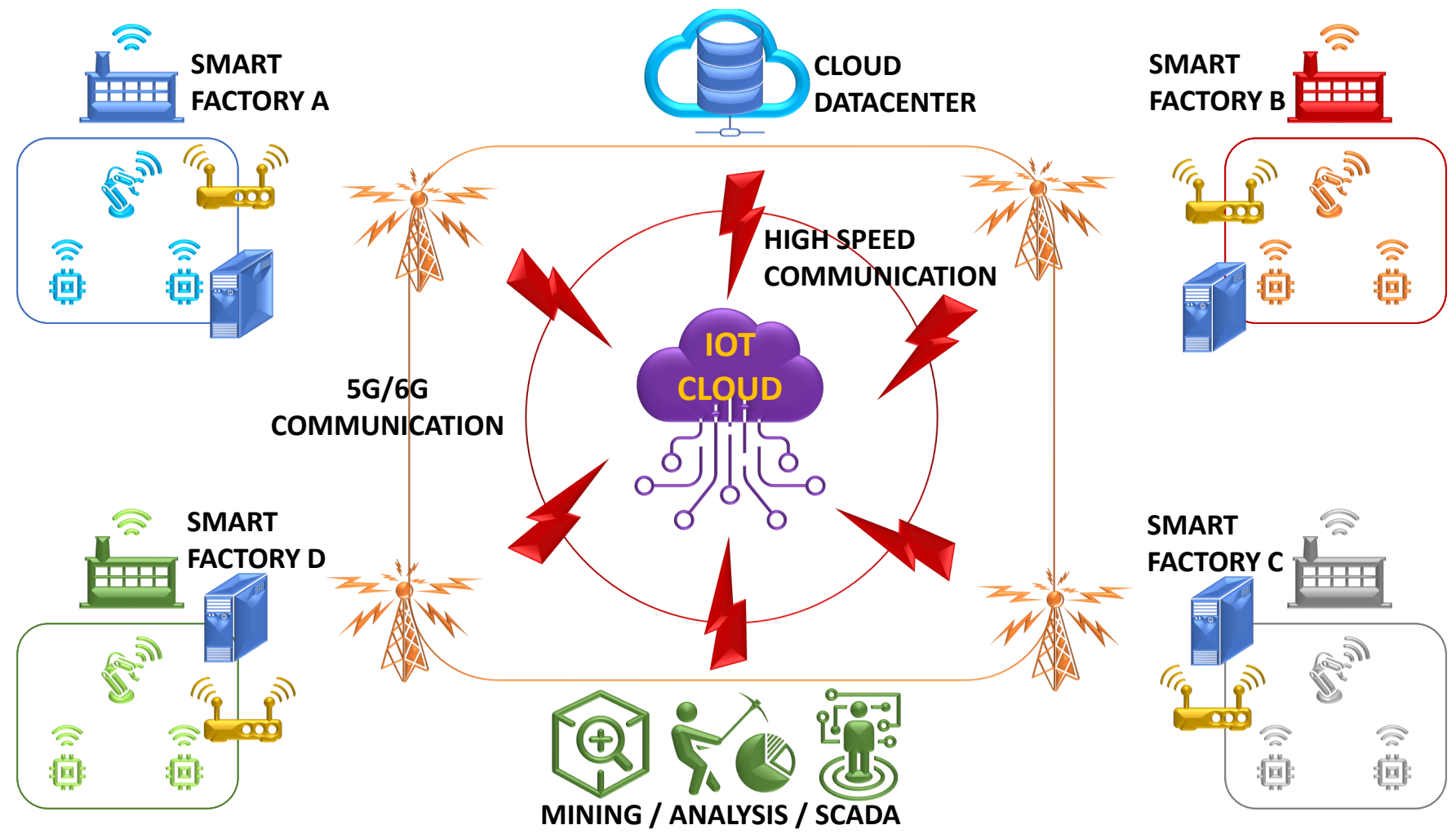

Fig. 9: Smart Factories In Today's World

Even in some areas, robots are intelligent enough to replace human supervision and understand situations using ML and AI techniques. This will help save human lives from facing dangerous situations, and robots will perform the tasks in such unfavorable circumstances. This will help in handling and managing disasters and incidents to respond effectively as well as efficiently. Another significant improvement is under progress in the usage of autonomous robotics in healthcare and surgery. Home maid tasks will automatically be shifted from humans to robots to view intelligence and context awareness being improved day by day. The useThe use of robotics for replacing human soldiers is also an active research area, but it is far out of this paper's scope.

3) Healthcare IOT: IoT technology has four main elements: 1) Internet, 2) Hardware, 3) Middleware, 4) Presentation. Any application proposal for SMART city must consider both policy and the technological challenges optimally favorable. It should not only during normal operations like parking, lighting, etc., but it should also be optimal for the data and systems in emergencies and disaster situations. [91] Proposed a conceptual mesh network for smart cities, in which devices will use their own stored energy during grid source. It has two modes; normal and emergency.

Healthcare management is vital to any future smart city [92]. City-level smart healthcare management components may include personal healthcare, smart hospital, telemedicine/online consultancy, smart medicine, smart disease control, and smart healthcare reporting. The first level of this system is the Personal Body Area Network with sensing devices to monitor a human body's various activities. These activities may include but are not limited to steps tracking, heart rate monitoring, blood oxygen level monitoring, and sleep monitoring [93]. These sensors are commonly referred to as the Healthcare IoT (H-IoT) [23]. Besides Covid-19 impacting businesses worldwide, the market of wearable devices is expected to see a rise of $14.5 \%$ till Q4 2020 and will continue the trend in upcoming years till 2024 [94]. Figure 12 presents the top 5 wearables companies' growth year by year.

Edge computing is patching low latency requirements between sensor and data processor by providing a shorter distance between the two and higher processing speed. Compiled, cleaned, and aggregated data, then forwarded to the cloud for intelligent decision making. Also, the H-IoT sensor generates enormous data, and its manual compilation is not worthwhile keeping in view the return on investment (RoI). ML and Big data analysis techniques are utilized in the current solutions to make the process more productive and in time [95].

Remote surgery is at the evolutionary edge where scientists and medical personals try their best to become free from geographical boundaries. The ultra-fast real-time network availability and IoT-based actuators with high-speed audiovisual aid technologies are helping in gradual progress in this field [96], [97]. Other healthcare aspects where next-generation technologies are being used include medical training, locomotive and sensory prosthesis, precision involve procedures, virtual reality and augmented reality (VR/AR) based rehabilitation and trauma rehabilitation [23].

A very comprehensive research analysis is presented in [98] in which the authors tested 100+ HIoT devices in order to evaluate security flaws ranging from unauthorized access, 


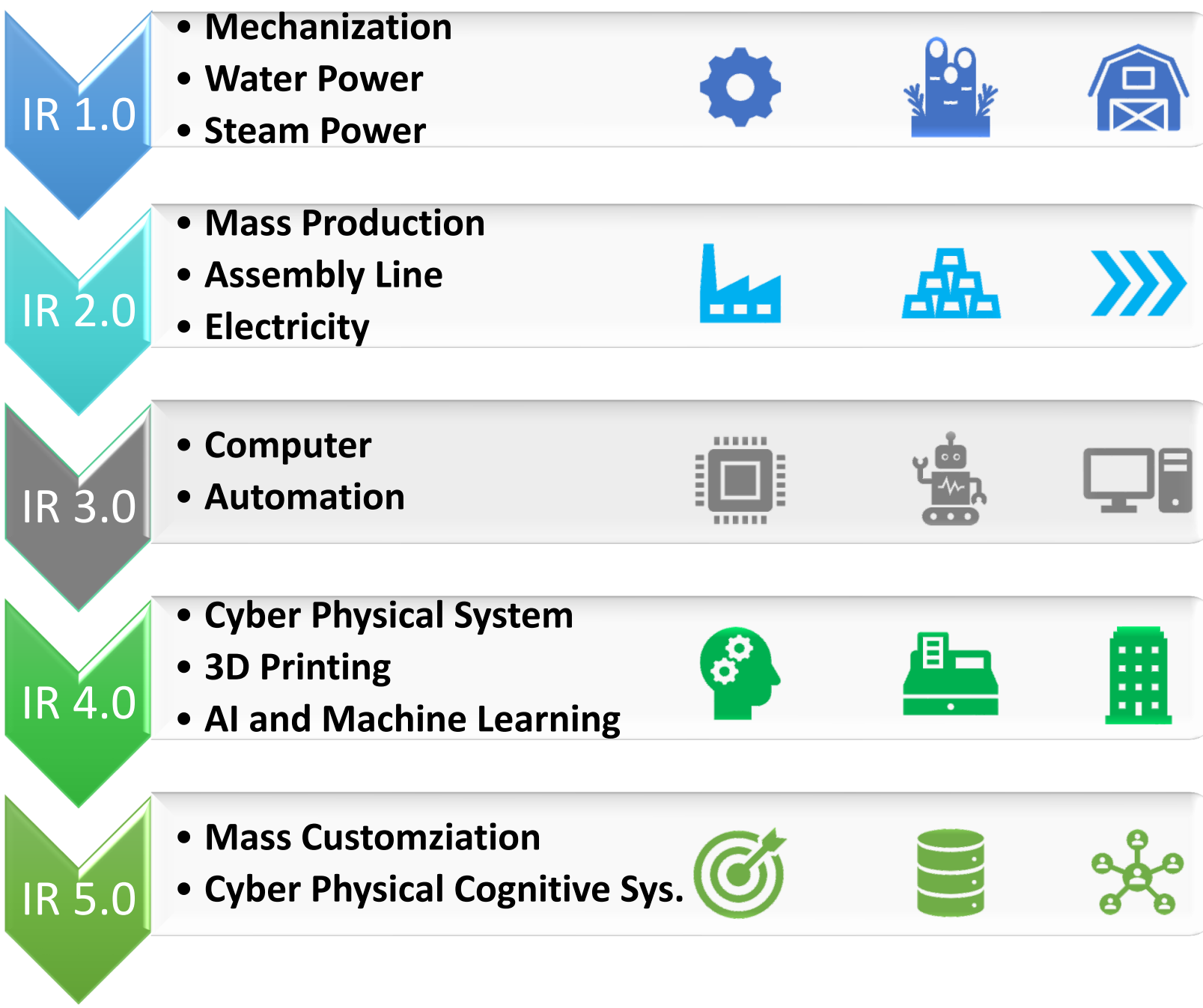

Fig. 10: Industrial Revolutions Chronology

TABLE IV: Summary of Cyber Physical Systems Literature

\begin{tabular}{|c|c|c|}
\hline Ref. & Proposed Work & Outcome \\
\hline$[63]$ & Presents a layered model for smart factories. & $\begin{array}{l}\text { The next generation smart factories are built on the layered model having an } \\
\text { End-user/management terminal layer, a cloud-based data analysis and decision } \\
\text { support layer, a network layer looking after all levels of communications, and } \\
\text { a physical resource layer managing the actual work done on the production } \\
\text { line. }\end{array}$ \\
\hline [64] & $\begin{array}{l}\text { Framework for the resiliency against accidents at- } \\
\text { tacks interaction. }\end{array}$ & $\begin{array}{l}\text { Present security framework for CPS for privacy issues, dependability of } \\
\text { components and layers, resiliency against accidents and attacks, interaction } \\
\text { among layers and components and their coordination, operational security, } \\
\text { system hardening, and most of the time, system availability. }\end{array}$ \\
\hline [67] & AI in Industrial Revolution 5.0. & $\begin{array}{l}\text { AI will be heavily used in Industrial Revolution } 5.0 \text { to change management, } \\
\text { transformation management, customer-centric production, and research and } \\
\text { development. }\end{array}$ \\
\hline [68] & Novel concept for HCPS. & Combines HI with trained AI solutions to bring the cognitive intelligent CPSs. \\
\hline$[\overline{72}]$ & $\begin{array}{l}\text { Interaction between the business, customer, and tech- } \\
\text { nology. }\end{array}$ & $\begin{array}{l}\text { Presented the framework for overlapping interaction between the business, } \\
\text { customer, and technology is the base of the industry } 5.0 \text { innovation. }\end{array}$ \\
\hline [79] & Robotics and automation technologies. & $\begin{array}{l}\text { Automation technologies are continuously used throughout the world in smart } \\
\text { cities. Robotics is a crucial part of this trend. Both of them have now become } \\
\text { natural partners in the cyber smart city arena. }\end{array}$ \\
\hline [65] & $\begin{array}{l}\text { The authors proposed how Tree Partitioning and } \\
\text { Deep Neural Network methods helped predict and } \\
\text { identify energy consumption. }\end{array}$ & $\begin{array}{l}\text { For the public sector, the authors present a framework of the intelligent system } \\
\text { (IS) for efficient Energy Management. In the proposed framework for data, } \\
\text { collection the authors used Big Data, IoT, and different ML methods. }\end{array}$ \\
\hline
\end{tabular}




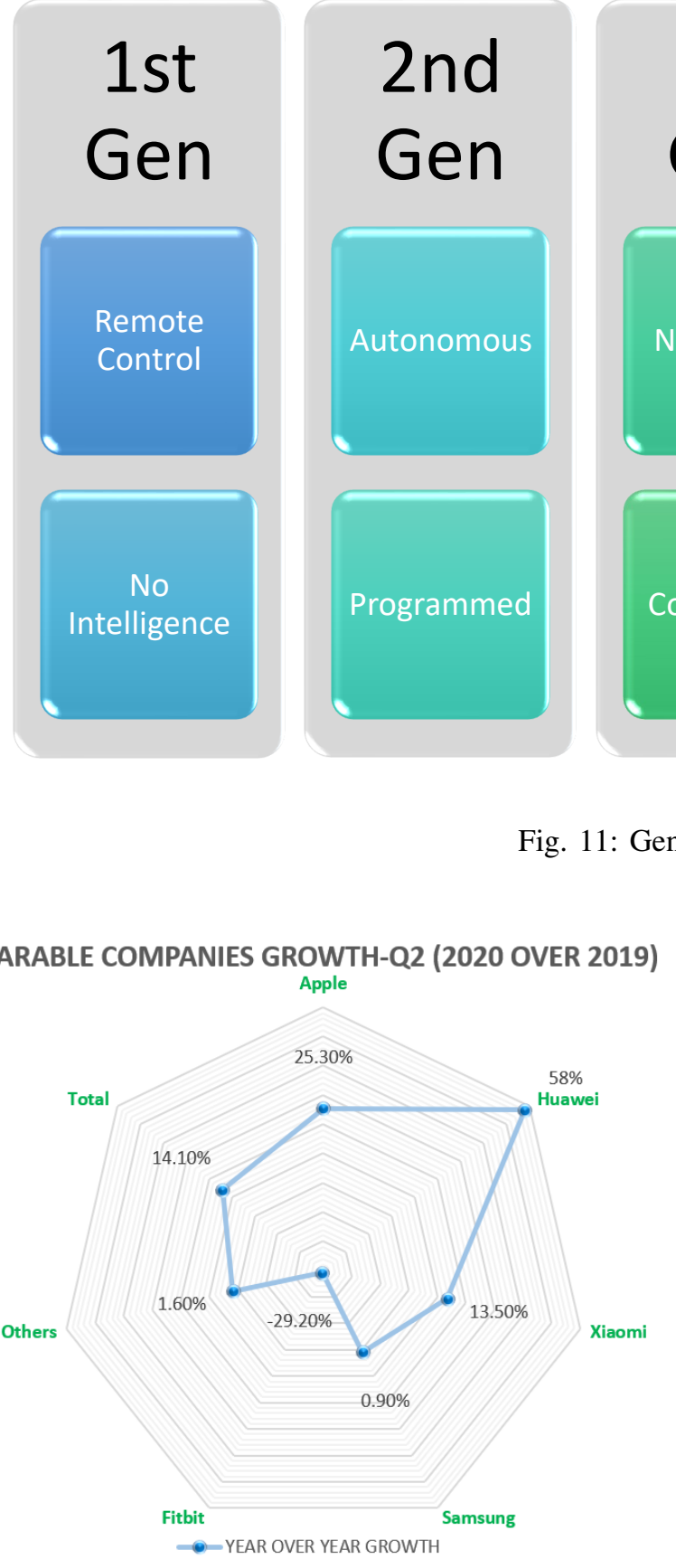

Fig. 12: Top 5 Wearable Companies Year-Over-Year Growth, Q2 2020

related footprints of different medical devices. Countermeasure to the typical flaws and policy-based control is also part of the solutions suggested treating the risks due to the security flaws.

Critical Analysis: IoT adoption in human life is so common nowadays that it becomes a part of our regular life. The advantages offered by HIoT. The advancements in the IoT devices, technology (communication and compute), power sources (usage and storage), and reduction in costs will make these devices come into typical humans' lives. Future smart cities will have a coordinated network of IoT devices in general IoT used for commercial and industrial purposes, smart home management, and personal health and fitness recording and management. Such devices will monitor the patterns of vitals related to the end-users norms and immediately identify the anomalies and update the local healthcare system to provide medical aid if requirements arise. Table IV presents the CPSs.

\section{Crisis Control Backup}

One of the essential aspects of smart cities' governance and management is planning, monitoring, and controlling the city's crisis. For this, different stakeholders, including healthcare, law enforcement, administration, disaster management, and other departments, are taken into on-board for immediate detection of threat, its information sharing to the different departments, planning the course of action, and then acting according to SOP's to contain the loss due to the situation.

For cities and states, crisis management is considered one of the essential tools, which can help them to stop spreading unwanted events to a larger scale [99]. Article [100] deals with developing a model of how to use knowledge in case of crisis management effectively.

1) Disaster Backup: Disaster management is the crucial aspect to be taken care of for any traditional and smart city. It becomes more critical to taken care of in a smart city as they are well organized and well managed, and all the actions are taken there are backed by the data. Planning and executing a disaster management operation in smart cities is relatively more straightforward than in traditional cities. The use of technology in the planning and management of disasters 
can increase human life savings and capital many folds [101]. United nation' office of coordination for humanitarian affairs (OCHA), in its strategic plan 2018-21, emphasized careful emergency response and disaster management in a wellcoordinated way to minimize the wide-scale losses a city at the national level, [102]. As per [103], humanitarian assistance and protection will be required for approx. Two hundred thirty-five million people in 2021. Figure 13 presents the components of proactive disaster planning.

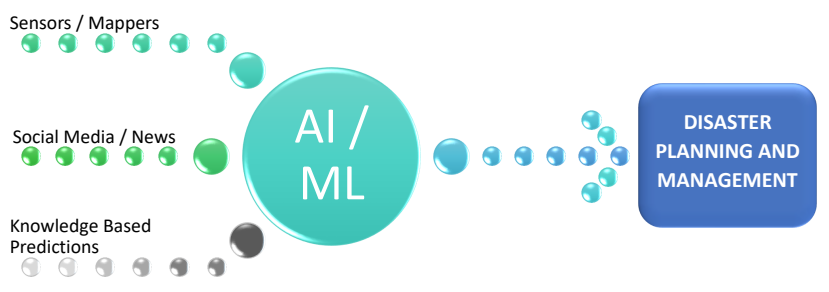

Fig. 13: Components of Proactive Disaster Planning

A proactive action is worth a lot more than the reactive action done afterward. Analysis of existing infrastructure to sustain against relevant disaster and community tolerance against services disruption during a disaster are two critical factors needed to study for such preventive and proactive management steps against disasters [104]. As the disaster planning is done by anticipating the expected disasters and planning accordingly long before the event's actual happening, there is ample time to review and gradually improve the planning. This planning not only focuses on the technology and infrastructure but also on the human preparation for facing disaster.

Factors that impact human behavior towards a disaster situation include previous disaster-facing experience (if any), demographics, culture, and awareness about the situation [105]. To prepare and rehearse people about the possible issue faced during expected disasters and interact with them. Emphasis on these impacts will bring fruitful results in handling the disaster and responding to it from the human side. Efforts on the community level always bring positive results in enhancing human capability in this regard [106].

Critical Analysis: As in smart cities, technological advancements exist at a high level; hence, disaster planning and management are based on data analysis. This data can provide complete insight into the issue and become a base for modeling, simulation, and making informed, intelligent decisions. Due to this, it is imperative to have a concise, compelling, targeted, and automated disaster response in current and future smart cities.

2) HVAC Empowerment: Human thermal comfort is one of the significant aspects of providing comfort to humans in daily life routines. Though, improving human efficiency in terms of productivity is the basic necessity of every work. However, providing a comfortable environment to workers has been turned out to be a significant improvement in work efficiency as well as stated by [107]. The authors in [108] proposed a novel approach of the adaptive HVAC control system in which the air conditioning systems regulate real- time through big data analytics. Their system monitors $\mathrm{CO} 2$ concentration, temperature, and threshold set by occupants for the optimal prediction of temperature for occupants. With the importance of HVAC in regulating human thermal comfort, it is also necessary to maintain the HVAC systems fit for the work as a state in [109]. The HVAC systems fault detection mechanism was based on data-driven techniques such as AI algorithms and traditional research skills. The cost-efficient adaptive HVAC approach proposed in [110] can work without the prior knowledge of cost expense data. The proposed HVAC management system uses real-time data and applies MultiAgent Deep Reinforcement Learning over the data [111]. However, an HVAC control system's relationship is also critically linked with parameters such as embodied carbon.

Critical Analysis: The reviewed studies prove that optimal HVAC is one of the vital necessities that should be present in every work or residential environment. The methodologies applied for the optimal HVAC environment are mainly based on automated systems that work on data obtained through human thermal comfort predictions or metrics obtained previously. The studies also state that the human work performance and efficiency also show a positive increment due to better human thermal comfort. Moreover, the reviewed studies also prove that the latest innovative methodologies automate the old conventional approaches, due to which less supervision and manual control are required.

3) Pantry Backup: Disasters due to calamities or any pandemic crisis, war, or crop shortage cause severe damage to the country's economy and affect the public on a massive scale. Shortage of food supplies will make the remaining supplies very costly and unreachable for the common public. This situation also leads to the decrements in the work efficiency of man force around the country. The article from New York Times [114] signifies the importance of pantry backup by highlighting that COVID-19 might kill the population with the virus rather than hunger. Authors in [113] studied the effect of COVID-19 on logistics systems and disruptions in the food supply chain. The authors developed a public distribution system (PDS) to encounter the problematic factors in a resilient supply chain during the pandemic. Their proposed system can provide predictive support to food supply chain vehicles to reroute if they encounter any restricted route. The food yield requirement is justified in [112] for the coming year. The authors clarify the food's unreported requirements and thus estimate the food's future needs for the world population. The authors in [115] performed a critical survey of the global food crisis due to pandemics and highlighted the future challenges.

Critical Analysis: The reviewed studies justify the need for pantry backup systems in multiple regions. Food is the fundamental necessity for human existence, and it is being ignored. In the coming times, the food crisis may lead to disasters of public infrastructure. The significance of pantry backup is highlighted in pandemic times, but it turns out more important in natural calamities and war times. The pantry backup system ensures the well defensive infrastructure to fulfill occupants' needs during tough conditions, making life comfortable and relaxed. It is an important factor of a future smart city. Table V presents Crisis Control Backup Literature 
TABLE V: Summary Crisis Control Backup Literature

\begin{tabular}{|c|c|c|}
\hline Ref. & Proposed Work & Outcome \\
\hline [99] & $\begin{array}{l}\text { This paper discusses the importance of smart traffic } \\
\text { management in the era of disaster and emergency } \\
\text { and how we can utilize smart transportation during } \\
\text { national and urban emergencies. }\end{array}$ & $\begin{array}{l}\text { The authors used an inductive research-based approach at the start. After } \\
\text { the careful analysis of the results of initial research by reviewing existing } \\
\text { crisis management systems and their approaches w.r.t smart trafficking and } \\
\text { transportation, a solution was proposed which can be merged in any crisis } \\
\text { management solution. }\end{array}$ \\
\hline [100] & $\begin{array}{l}\text { The article is based on the consideration of collabo- } \\
\text { ration and data management issues in the crisis. }\end{array}$ & $\begin{array}{l}\text { A meta-model was presented in the paper that acts as an information basis for } \\
\text { other developed tools for crisis management and collaboration. }\end{array}$ \\
\hline [101] & $\begin{array}{l}\text { Due to the increase in the frequency of natural and } \\
\text { human-made disasters, relief operations saw a rising } \\
\text { trend in the near past. This paper sheds light on } \\
\text { the ICT and disaster/relief information linkages and } \\
\text { importance. }\end{array}$ & $\begin{array}{l}\text { The proposed solution is based on four main components of the city's disaster } \\
\text { management: dynamic network analysis, data integration and data analytic, } \\
\text { multi-data sensing, and multi-actor game-theoretic decision-making. }\end{array}$ \\
\hline$|\overline{104}|$ & $\begin{array}{l}\text { Disturbance/unavailability of critical infrastructure } \\
\text { and services during/after the disaster results in dam- } \\
\text { age and slowdowns the rehabilitation efforts. Re- } \\
\text { silience to such events eventually results in better } \\
\text { disaster management. }\end{array}$ & $\begin{array}{l}\text { The proposed framework analyses the community's vulnerabilities that may } \\
\text { damage critical infrastructure or unavailability of its services. A disruption } \\
\text { tolerance index (DTI) is then calculated to quantify the impact mainly on } \\
\text { healthcare facilities. }\end{array}$ \\
\hline 107 & $\begin{array}{l}\text { The article is about the increasing usage of IoT } \\
\text { devices in controlling, managing, and sensing the } \\
\text { heating, ventilation, and air conditioning of smart } \\
\text { buildings and their impact and efficiencies. }\end{array}$ & $\begin{array}{l}\text { The paper presents Personalized Comfort, the novel model that predicts the } \\
\text { thermal sensation. A publically available dataset ASHRAE RP- } 884 \text { was used } \\
\text { for analysis. ML and DL models were applied to predict the thermal sensation } \\
\text { using the proposed model with attaining accuracy of above } 85 \text { percent. }\end{array}$ \\
\hline$|\overline{110}|$ & $\begin{array}{l}\text { HVAC usually consumes } 40-50 \text { percent of the cost } \\
\text { of energy which becomes a burden on operations. } \\
\text { Minimizing this cost can be very beneficial overall. }\end{array}$ & $\begin{array}{l}\text { This paper presents a model based on the Markov game without having prior } \\
\text { knowledge of the building's thermal dynamics. It can control the HVAC based } \\
\text { on multi-agent deep reinforcement learning with an attention mechanism. }\end{array}$ \\
\hline$|\overline{112}|$ & $\begin{array}{l}\text { The growing concern in the agriculture field world- } \\
\text { wide is the expected increasing food deficit. UN's } \\
\text { food and agriculture organization (FAO) is closely } \\
\text { monitoring and working on the issue. }\end{array}$ & $\begin{array}{l}\text { This article provides the depth analysis of the current situation of this food- } \\
\text { deficit crisis. The article states the major issue faced worldwide and the effort } \\
\text { to fight against hunger and feed humans throughout the world. }\end{array}$ \\
\hline$[\overline{113]}$ & $\begin{array}{l}\text { The Covid-19 virus impacted the lives of humans and } \\
\text { crippled the healthcare systems, and severely churns } \\
\text { out the economies. The global food supply chain is } \\
\text { also one of the major areas that face its worst impact. }\end{array}$ & $\begin{array}{l}\text { Due to the restriction imposed on the global and country/city level, the supply } \\
\text { chain of food items got disturbed and disrupted. The study provides a simulated } \\
\text { model to output a resilient supply chain model to the restriction and can provide } \\
\text { alternate routes and measures to imposition travel restrictions in specific areas. }\end{array}$ \\
\hline
\end{tabular}

section tabular. Figure 14 presents ML and AI relationship.

\section{Artificial Intelligence}

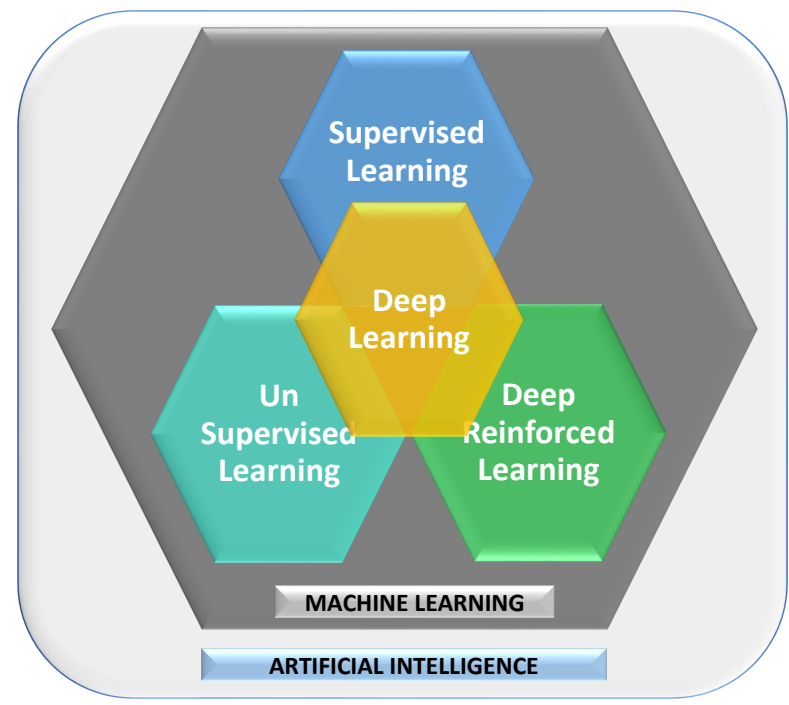

Fig. 14: Machine Learning and Artificial Intelligence

Keeping in view the trend of urbanization in the population, it is anticipated that in 2050, 60 to 70 percent of the world's population will shift to the urban areas [116]. To cater to this enormous shift and issues created with this population transfer, the first world countries started incorporating the latest technologies in the infrastructure and management of the cities. Due to the data-driven nature of the fourth industrial revolution technologies, their incorporation results in massive data. The interconnected technologies and their reliance on each other's output to take intelligent and data-centric decisions are handled by heavy usage of ML techniques and AI [117].

The current smart cities see a trend of such incorporation. Various cities are on the way to achieve intelligent smartness in various city's aspects [118]-[123]. The typical issues faced include but not limited to pollution and degradation of the environmental quality, shortage of resources as compared to the need of the population, traffic jams, higher cost of living, decrease in living standards, higher poverty rate and other social issues [124]. The city transformation project planning also requires careful planning, keeping in view the digitization and technical aspects and considering an unexpected spike in the population due to urbanization [125].

According to [126], the current status of smart cities throughout the world is divided into three tiers, with "model smart cities" at tier 1 followed by tier 2 and tier 3 smart cities as "follower smart cities." The primary differentiation role between model and follower is advanced ML and AI techniques for overall management and decision-making.

In future smart cities, the most usual tasks and processes will be defined and managed automatically using artificial analysis. This will help in the synergy of system operations, bringing timely execution of the functions in different aspects of the smart city and providing a reliable living experience. 
Requirements generations in smart city scenarios bring advancements in AI techniques to fulfill those requirements. The same advancements in AI also generated further new requirements in the smart cities functions, which are a candidate to be solved using those techniques. Hence, both AI and smart cities' impact results in a win-win situation where both help each other excel in their respective fields while supporting advancements in each other.

In a smart city of the future, the autonomous processing of information and smart decision-making based on AI-based ML and behavior analysis will be the major part of city governance and management. Human capabilities will be used for high-level decision-making and management. Day-to-day operations will be automated and reliably handled by the computer brains, which will act on competent will within the thresholds as per the system designers' feeding.

1) Machine Learning: The dawn of AI has changed conventional methods into robust automated methods. ML is that branch of AI that deals with the automation of human-based tasks by training the model over specific data and then testing the trained model over the unseen data to get the predicted results [127]. AI plays a vital role in revolutionizing smart cities. There are tremendous applications of ML developed in smart cities. Few of the major applications are stated by [117] which includes intelligent transportation systems (ITSs), cyber-security, smart grids (SGs), UAVs, 5G, and B5G communications, and smart health care system in a smart city. The authors also highlighted the upcoming challenges in future research directions [128]. The authors in [129] proposed an ML-based approach for smart cities to make energy consumption efficient in terms of cost by providing the prediction of optimal configuration with the data collected by IoT network and Big Data collections. The authors prescribe implementing this model in public sectors' business intelligence systems to predict investments in reconstruction measures. The authors in [130] surveyed the capabilities of data mining and ML techniques in the classification of network traffics. Their deep evaluation of the ML approaches also highlights the future perspectives of ML applications for sustainable smart cities. Iskandaryan et al. worked on the living environmental quality analysis. They evaluated the ML algorithms to examine the air pollution extent with sensors data [131]. The applications of ML in smart cities are not limited to automating human-based work only. Rather their applications are extensively found in the field of medicine and safety precaution systems as well. The authors in [132] proposed a ML approach to detect the COVID-19 spread across the smart cities. Their study also covers the research areas of predicting the next epidemic, effective contact tracing, diagnose COVID-19 cases, monitor COVID-19 patients, COVID-19 vaccine development, tracking potential COVID-19 patients, and aiding in COVID-19 drug discovery. It provides a better understanding of the virus in smart cities through ML approaches. The authors in [133][138] proposed ML-based state-of-the-art solutions for prevention of cyber attacks in smart cities. However, it is imperative to carefully bring this intelligence and automation to save from the possible negative impacts of the autonomous systems' automated decision in the absence of emotions and contextual intelligence [139]. Figure 15 presents different techniques of ML. CPS security, which is the new, smart and intelligent critical infrastructure-based system. It can be secure using ML and the challenges, solutions proposed in published research. ML and future research directions in resiliency in ML, context awareness in ML, federated learning, and minimizing false positives and false negatives while detection threats to the CPS are covered comprehensively in the paper [140]. Another study [141] focused on practical aspects of cybersecurity incidents detection, analysis, and classification using ML techniques which can be administered in the scenario of smart cities.

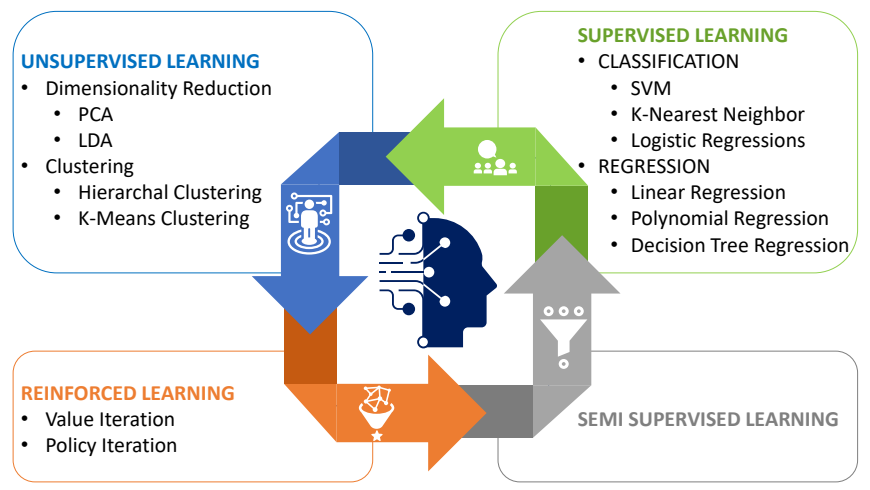

Fig. 15: Machine Learning Techniques

Critical Analysis: The reviewed studies highlight the significance of AI applications in smart cities. Nevertheless, the ML approaches are considered to be the fundamental pillar of a smart city. The ML approaches automate the conventional tasks and provide solutions to complex problems to be monitored through humans. The intelligence brought to the systems must have a cap over them to control the autonomous decisions under human supervision. Though advances bring contextual and situation awareness into automated decisionmaking, carefully supervised learning is always a need to protect from the possible harm due to the machine itself is factual decision-making. Defining a boundary to limit the machine's autonomous decision is a key challenge. The balance between automation and human control is the next big thing for future smart cities' technological advancements.

2) Deep Learning: Deep learning is the advanced branch of AI that consists of neural networks [142]. These neural networks are composed of multiple hidden layers, making them superior classifiers and predictors [143]. There are tremendous applications of DL algorithms in modern smart cities as these algorithms can solve the task with more capabilities and proficiency. The authors in [17], [19] reviewed the DL application in future smart cities in depth. The authors surveyed topics that include urban modeling for smart cities, smart cities' intelligent infrastructure, smart mobility, transportation, smart urban governance, resilience and sustainability smart cities, smart education, and smart health solutions. With the development of smart devices and inter-connectivity through IoT, Many concerns relevant to cyber attacks and privacy issues have been raised. The DL algorithms being considered for their supreme capability of anomaly detection and classification encounter cyber-threats with high proficiency. The researchers in [144]- 
[151] proposed several DL algorithm based methods to detect and encounter the cyber-attack over the smart cities IoT based infrastructure. These studies also highlight future research directions by proposing the comparative analysis between DL algorithms regarding their accuracy.

The authors in [152] proposed a DL algorithm for energy demand forecasting. The system's primary function is to analyze the energy demands and consumption and optimize the energy usage to make the energy consumption optimal in terms of cost and efficiency. The authors in [153] highlighted a drawback of air pollution due to cities' modernization. The industrial wastage and production of non-eco-friendly materials are causing the air to be polluted on a dangerous scale. The proposed system is based on a DL algorithm that analyzes the sensor's data to predict whether the air pollution at a certain place is increasing or not. Thus it indicates at earliest to control the pollution. This methodology is considered beneficial for smart city residents since it makes the environment safer and healthier for the occupants. DL applications are widely observed in modern smart cities in which disaster management is the primary one. The authors in [152] proposed a DL based system for smart cities to manage the disaster by predicting the optimal evacuation plans and traffic management strategies. Moreover, DL algorithms have proficient capabilities in manipulating tasks relevant to image processing. The authors in [154]-[157] devised state-of-theart researches related to image processing task by using DL. The researchers in [158] highlighted the usage of DL in smart cities' healthcare sector.

Due to the steep increase in usage and deployment of IoT devices in every industry, the security challenges related to IoT have become an integral part of these systems. Ensuring the security with such a large number of active IoT devices with veracity and variability in architecture, methodologies, interoperability, supported methods, and update cycles, it is almost impossible to identify security issues using traditional/manual methods. This concept was taken up in [159] to use power analytical methods provided by ML and DL to enhance IoT security at a large scale. The smart city has many components based on standard services like power, network, log correlation, access control, etc. Among them, the vital part played is by power and then by communication infrastructure. The heavy reliance on smart city infrastructure on intelligent data-based decisions makes communication an essential entity for the whole operation. Use of reinforcement learning w.r.t communication within large-scale deployment like smart cities are discussed in [160] where authors present application of deep reinforcement learning in applied scenarios of network and communication. Merging DL in an IoT environment can give better results-oriented benefits; however, challenges like low computational and power resources are yet to solve in this regard [161].

Critical Analysis: The reviewed studies conclude that DL robustness in revolutionizing conventional tasks is a great fortune for humankind. These algorithms automate tasks and solve daily life problems with proficient accuracy than a human. Though, there are several other factors too that revolutionize smart cities. Nevertheless, DL applications have grown to show great interoperability with other technologies that make DL a significant component for future smart cities.

3) Computer Vision: Computer vision is a multidisciplinary approach towards acquiring, processing, analyzing, and understanding the images as per the defined contexts and requirements [162]. The process involves the usage of optics to acquire the image using digital/smart cameras using the biological vision patterns used by the human eye, applying mathematics, statistics and geometry for non-linear, multivariable signal processing to optimized and analyze the acquired image, applying ML techniques to understand the image cognitively using the application of advance AI and then utilize the resulted knowledge in order to take actions in the real world or for automatic robot control. Figure 16 presents the computer vision characteristics.

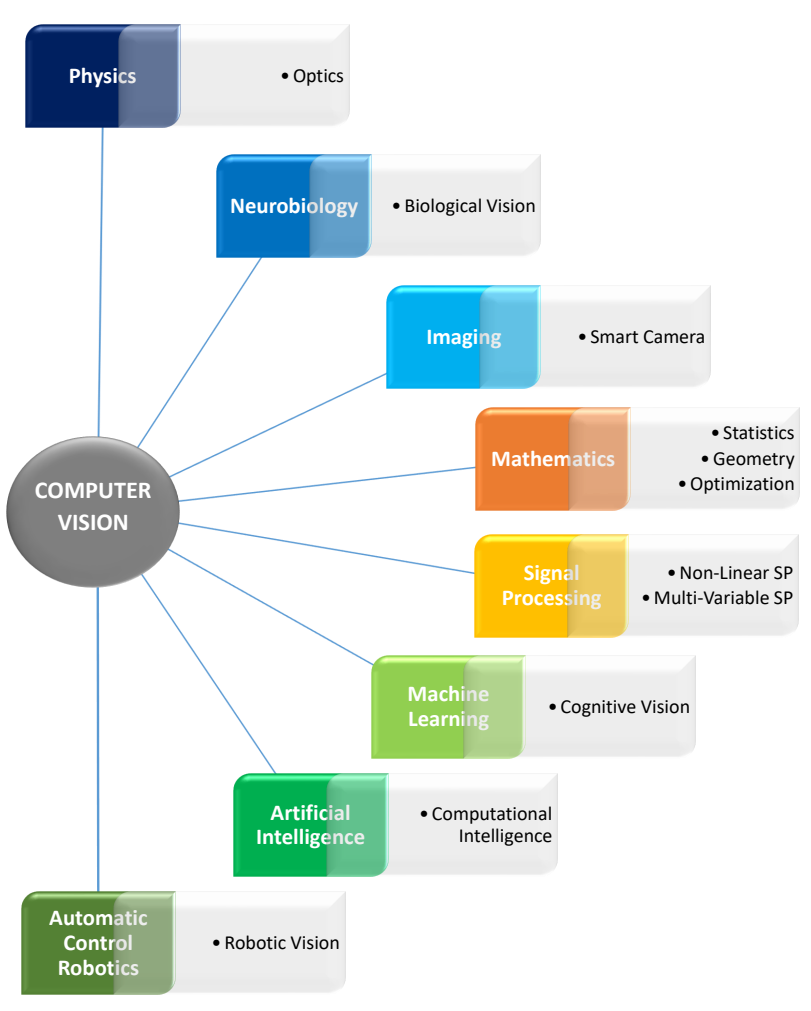

Fig. 16: Computer Vision

Computer vision has a very critical role in smart cities' various functions. The most important computer vision implementation is in the monitoring domain, which intelligently takes care of the issues and abnormalities in smart cities' various functions. Example usage of computer vision in the smart city scenario was discussed in [163] regarding traffic and lane monitoring using smart traffic infrastructure. In [164], the authors discussed various practical aspects concerning computer vision usage in multi-camera vehicle re-identification and tracking, vehicle counting, and traffic anomalies detection. A DL approach was presented to detect an object in real-time in $2 \mathrm{D}$ and $3 \mathrm{D}$ in [165]. Another very summarized discussion 
was presented in [166] regarding usage of DL techniques on the data gathered by IoT based sensors deployed at a city scale for the modeling/analysis of various aspects of smart cities like traffic, healthcare, urban governance, education drainage of waste, movement of the citizen. Advanced computer vision can be used further for medical imaging, bringing paperless transaction management in government offices, commercial organizations and financial institutions, medical imaging, urban planning, crowd control, human and assets tracking, and other allied areas. Another practical monitoring framework was proposed in [167] to utilize computer vision techniques to assess distance monitoring, crowd monitoring, physical contact monitoring, and symptoms highlighting as per SOPs Covid-19 in the smart city. The multilevel information fusion edge architecture (MELINDA) was proposed and discussed in [168], and [169] further shed light on bringing the video frame processing on the edge computing keeping in view the increased load on server processing in a well-monitored smart city environment. An advanced DL based office equipment occupancy analysis techniques are discussed in [170] to make intelligent HVAC requirement analysis and control as per the anticipated cooling requirement.

\section{Critical Analysis:}

Computer vision will have a crucial role in future smart cities. It will eventually become the backbone for intelligent monitoring, control, and decision-making in many aspects of a smart city. The application of computer vision in smart manufacturing, smart crime monitoring, safe city management, automated vehicle monitoring, smart road infrastructure, law enforcement activities, business and offices management, smart home, urban planning, smart transportation using smart cars, disaster management, robotics, and healthcare will eventually result in bringing intelligence and automation to the allied tasks. Table VI presents the AI Literature section in tabular form.

\section{E. Future Transportation}

Cloud-based technologies are in use to make the road infrastructure safe and secure for commuters. The roadside infrastructure uses sensors including pressure detectors, inductive loop detectors, microwave detectors, image detectors, ultrasonic detectors, and infrared detectors to detects the objects, their position, and their speed to perform complex calculation and then communicated the results to other components of the infrastructure as well as to smart vehicles traveling on the infrastructure [171].

The overall smart road infrastructure usually provides services including real-time monitoring, real-time information, journey planning, and guidance, intelligent road lightening system, smart road signaling system, road traffic load management, road lane management based on the load, smart ticketing and violation identification, and ticketing, an electronic payment system for toll charges as well as fine payments, speed and environment management as well as an alert system, parking management and guidance, incidents and accidents response, control and emergency system management [172]. In the future, smart unmanned vehicle management, smart fleet monitoring, identification, and cross border traffic management are the key systems to join the list. Figure 17 presents the smart transport infrastructure.

Situational awareness is the main point on which the whole smart road infrastructure works. The system's components make a model at the current point of time for the current point of a place containing the real-time data and then transfer it to other components/subscribers before calculating the updated model at the next point of time. Other stack-holders of the system then receive the model using high-speed communication channels like 5G and others and utilize this information to further utilized in calculating point of time model w.r.t their position within the infrastructure [173]. After calculation, the model was used to decide and give necessary information back to the system to other components to calculate their point of time models.

The key components of smart next-generation transportation for future smart cities rely heavily on power usage, which is also researched for shifting its generation to clean and green energy sources. The researcher in [174] divides the outcome of their papers into the following four classifications based on their usage and impact. They are power, communication, computer, and security. These classes of future smart transportation are discussed briefly, focusing on interdisciplinary management of energy and information related to infrastructure.

1) Autonomous Vehicles: The smart transportation aspect in the smart city has a unique feature of inter/intra vehicle communication consists of the vehicle to infrastructure (V2I), vehicle to pedestrian (V2P), vehicle to vehicle (V2V), vehicle to sensors (V2S), vehicle to cloud (V2C) and vehicle to home $(\mathrm{V} 2 \mathrm{H})$ [175]. If done in an organized way, this communication can help solve the city's transportation infrastructure problems. Figure 20 presents vehicle to everything (V2X) characteristics. Authors of [176] performed an in-depth review of communication technologies challenges and solutions concerning the physical layer of communication, security, synchronization issues, MBMS, and management of resources in a V2X network backed by LTE infrastructure. The solutions were analyzed for the scenarios like availability of multiple communication service providers, availability of single communication service providers, or the case when the vehicles are out of the coverage area of communication providers.

The coordinated communication for transportation infrastructure can play a vital role in smart cities' traffic problems, tested by partial deployments in the world's megacities. Figure 18 presents the overview of the communication structure of a smart vehicle. Around 1000 cities worldwide are trying to implement smart city structures within them, and more than half of these cities are in China [177]. This rapid engagement of smart initiatives also forces the allied industries like vehicle manufacturers to start providing the intelligence and support of smart city infrastructures in their vehicles. Initially limited to the drive support systems, this support now enhances their scope to go for a driver-less experience. Future smart cities will see self-driving vehicles very soon. Smart autonomous vehicles will make it possible to commute more safely and effortlessly while enjoying driver-less experience [178]. The 
TABLE VI: Summary of Artificial Intelligence Literature

\begin{tabular}{|c|c|c|}
\hline Ref. & Proposed Work & Outcome \\
\hline $\mid \overline{116}$ & $\begin{array}{l}\text { Designed an energy management system (EMS) for } \\
\text { IoT based infrastructure for edge computing. }\end{array}$ & $\begin{array}{l}\text { The authors described the overview of the IoT infrastructure based on the EMS, } \\
\text { proposed IoT based framework, and software system with edge computing. The } \\
\text { paper presents a scheduling scheme based on efficient energy for the proposed } \\
\text { framework and software using the deep reinforcement learning method. Finally, } \\
\text { the authors described the effectiveness of the presented framework. }\end{array}$ \\
\hline 117 & $\begin{array}{l}\text { The author's primary purpose is to explore the role } \\
\text { of ML, AI, and DL in the development of Smart } \\
\text { Cities. }\end{array}$ & $\begin{array}{l}\text { In this survey paper, the authors present details of different applications prior } \\
\text { techniques in smart cities; cybersecurity, smart health care system (SHCS), } \\
\text { intelligent transportation systems, operative use of the UAVs and the effective } \\
\text { services of } 5 \mathrm{G} \text { communications, and efficient energy utilization in the city SG. } \\
\text { At the end of the survey, the authors present research challenges and future } \\
\text { directions for smart cities. }\end{array}$ \\
\hline$|129|$ & $\begin{array}{l}\text { The authors proposed how tree partitioning and deep } \\
\text { neural network methods helped predict and identify } \\
\text { energy consumption. }\end{array}$ & $\begin{array}{l}\text { For the public sector, the authors present a framework of IS for efficient energy } \\
\text { management. The authors used big data, IoT, and different ML methods in the } \\
\text { proposed framework for data collection. }\end{array}$ \\
\hline$|\overline{130}|$ & $\begin{array}{l}\text { For sustainable smart cities, the authors present ML } \\
\text { and Data Mining methods for traffic classification. }\end{array}$ & $\begin{array}{l}\text { The authors present ML and data mining methods for traffic classification for } \\
\text { sustainable smart cities. }\end{array}$ \\
\hline $\mid \overline{142}$ & $\begin{array}{l}\text { The authors presented the deep ANN method for } \\
\text { defending Android malware. }\end{array}$ & $\begin{array}{l}\text { The authors assessed the efficiency of the Deep ANN method with traditional } \\
\text { classifiers. The authors find that the method is best on static layer category, } \\
\text { family, and classification accuracy malware. }\end{array}$ \\
\hline$|\overline{152}|$ & $\begin{array}{l}\text { The authors proposed a framework for GPU-based } \\
\text { DL and big data management and computations. }\end{array}$ & $\begin{array}{l}\text { In disaster and evacuation situations, the DL approach results are very effective } \\
\text { in predicting traffic behavior. For disaster management, the proposed framework } \\
\text { is the first that combined different technologies like in-memory and data-driven } \\
\text { computations, DL, and GPU. }\end{array}$ \\
\hline$|\overline{162}|$ & $\begin{array}{l}\text { The authors provide a new method for real-time } \\
\text { problem solving for digital forensics investigators for } \\
\text { investigating the suspect machine. }\end{array}$ & $\begin{array}{l}\text { The proposed method collects image data at the byte layer from the acquired } \\
\text { machine and performed accurate and fast image detection using a DL algorithm, } \\
\text { and separates suspicious images that can be used as evidence in a case. The } \\
\text { proposed method identified suspicious images and flagged them. In terms of } \\
\text { time and performance, automated image detection is vital in digital forensics. }\end{array}$ \\
\hline $\mid \overline{163}$ & $\begin{array}{l}\text { The proposed study aims to give an essential so- } \\
\text { lution for continuous land acquisition to overcome } \\
\text { increasing traffic using the encouraging application } \\
\text { of flexible lane dividers to apply in the smart cities } \\
\text { environment. }\end{array}$ & $\begin{array}{l}\text { The authors provide a lane span that handles the width of the road, evading the } \\
\text { necessity of road development. The video-based data is collected from placed } \\
\text { cameras alongside the single stretch and examined in real-time for decision. }\end{array}$ \\
\hline
\end{tabular}

adoption of self-driving cars by the general public will increase with the advancement and incorporation of such technologies in the infrastructure of smart cities. Situational awareness, route planning, drive control, and actuator management are the topics on which currently published literature is reviewed in [179]. The paper covers state-of-the-art technologies, design and implementation issues, and future challenges w.r.t autonomous vehicles.

Critical Analysis: A driverless, self-running, and autonomous transport system is the need of the future smart cities. Researches on the multiple aspects of autonomous vehicles are in full swing: complexity calculation, risk identification, scenario analysis, and accurate and real-time decision making. While propagating the decision to the other vehicular nodes around to help them make an informed decision and doing all this under the strict control of transport infrastructure rules and control applied on the location of presence are the emerging challenges to tackle appropriately. Table VII presents the future Transportation Literature section in tabular form.

2) Hydrogen Fuel Cars: Alternate energy consideration for transportation is an area of research for decades. After because currently, forms of fuels are limited, many alternated were considered fuel giving the same performance with a lesser change in engine technology and cost-effectiveness. In power automobiles, hydrogen cells with hydrogen (h2) can be utilized as fuel, which proves effective and economical along with lesser greenhouse impact than traditional gasoline-based fuels
[184]. The demand for hydrogen is increasing globally year by year, showing the increase in hydrogen usage as a fuel for automotive and other industries [185]. As per [180], the global fuel cell market, whose site was USD 342 million in 2019, will be anticipated to reach USD 1059 million by 2024 positively. Figure 19 presents the year-wise global hydrogen demand.

However, there are barriers like high initial costs, relatively lesser lifetime, and lack of hydrogen fuel refueling infrastructure, which are preventing the broader adoption of this energy-efficient and climate-friendly alternate fueling system by the masses [186]. The user acceptance for such conversion of fuel source mainly depends on the cost and performance optimization of hydrogen fuel cells, which still have enough room for research [181].

Critical Analysis: Alternate fueling for transportation infrastructure is a hot research topic. Keeping in view the gradually depleting fossil fuel reservoirs and their negative impacts on the environment are forcing the authors to look for alternate sources to fulfill the fuel requirements of transport infrastructure.

3) Flying Cars: Traffic congestion issue is a significant concern for the big cities management. The more populated the city becomes, the more commuters come on the road and exerts a load on the transportation infrastructure. Careful planning is a crucial factor used to distribute the traffic load by placing commercial hubs and areas in intelligently selected 


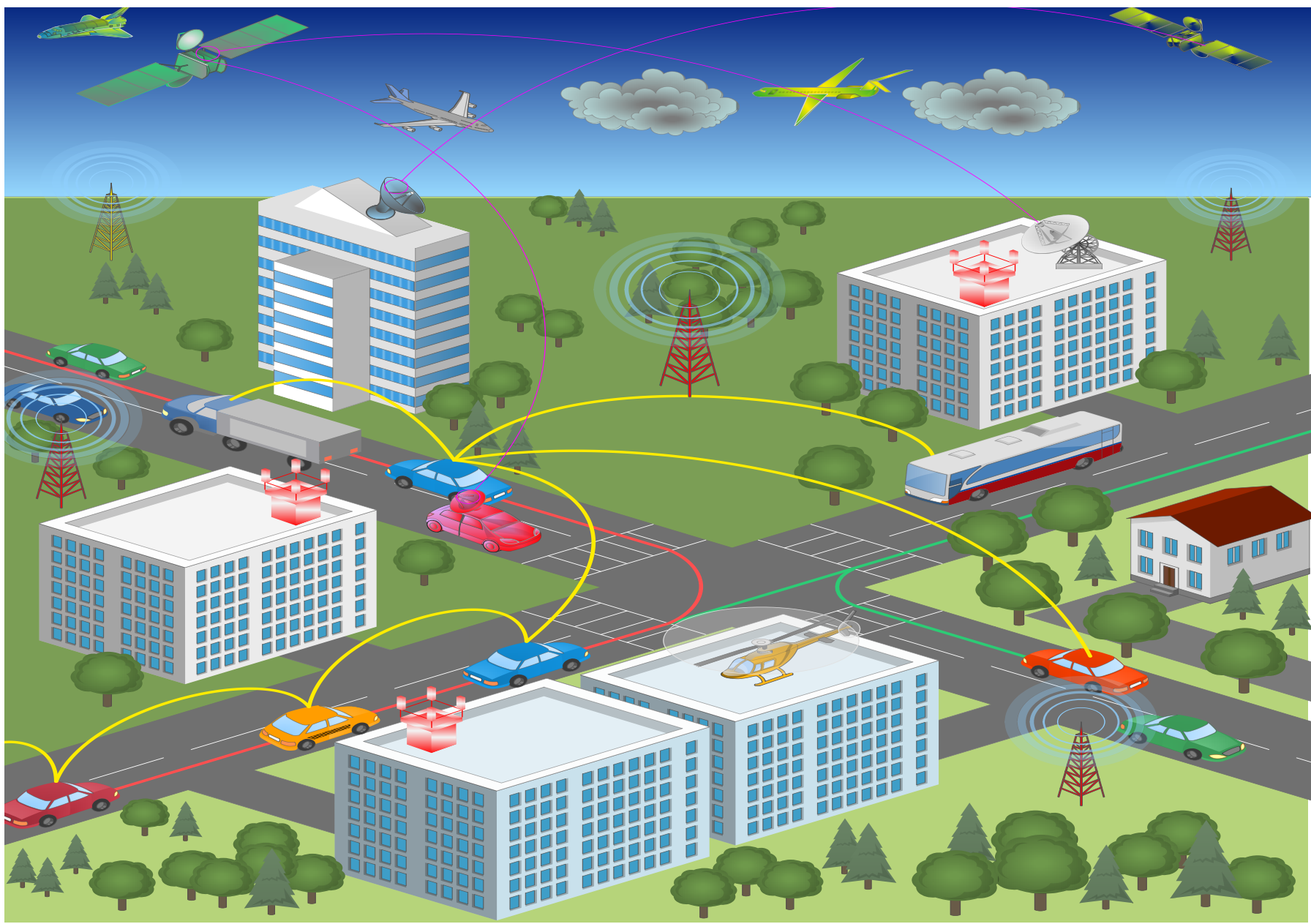

Fig. 17: Smart Transport Infrastructure

points for smooth traffic flow. A new paradigm of the solution in this regard is the use of flying cars. The use of flying cars (obviously under meticulous planning and control) can provide an efficient and faster solution to this issue [187]. A recent study in this regard [188] which was focused on agent-based approach, finds out that trip origin/destination points, average distances traveled in the urban contexts, and the transition location nodes are the main factors that impacts travel cost. Making such travel cost-effective and bearable is a critical challenge in making further progress in this regard. The significant challenges in this domain include safety of the overall transport infrastructure, training certification, and worldwide standardization in the domain of pilot, as well as cars manufacturing, flying traffic rules and regulations, environmental impacts, logistics and sustainability, human adoption, and cybersecurity [182]. Each aspect of flying cars' infrastructure development and implementation requires deep and intensive research to produce a well-designed, implemented, and cyber-safe, cost-effective, and easily adaptable system for real success. Some online store giants are already integrating drones for the delivery of goods to customers. Amazon's Prime Delivery is an example where amazon will deliver the parcel up to $5 \mathrm{~kg}$ within 15 miles radius in 30 minutes after order [189]. It is not very far in the future if the technology adoption trends go at the same pace that we will see the flying cars available as ride-sharing services for a common commuter [183].

The challenges mentioned in [190] though being targeted on UAVs are pretty aligned with the challenges and issues in the scenario of the flying car with little or no modification like heterogeneous network convergence, computation management and offloading, resource scheduling, energy conservation, security, and performance. To secure autonomous flying vehicles like drones, a certificate-based access control framework is proposed in [191] which is designed to provide security while keeping the efficiencies in computing and power consumption.

Critical Analysis: Flying cars is a dream which is near to come true. Recent experiments in the UAE for flying support by law enforcement personals and flying delivery services by amazon are examples of practical experiments in this regard. However, the area has much room for improvements in the definition of laws and protocols of traveling, air traffic infrastructure, control mechanisms, and flying vehicle secure design and operation. Flying transportation will be a premium addition to the already advanced level technologybased facilitation provided in the future's smart cities. The purely technical infrastructure already available for future 


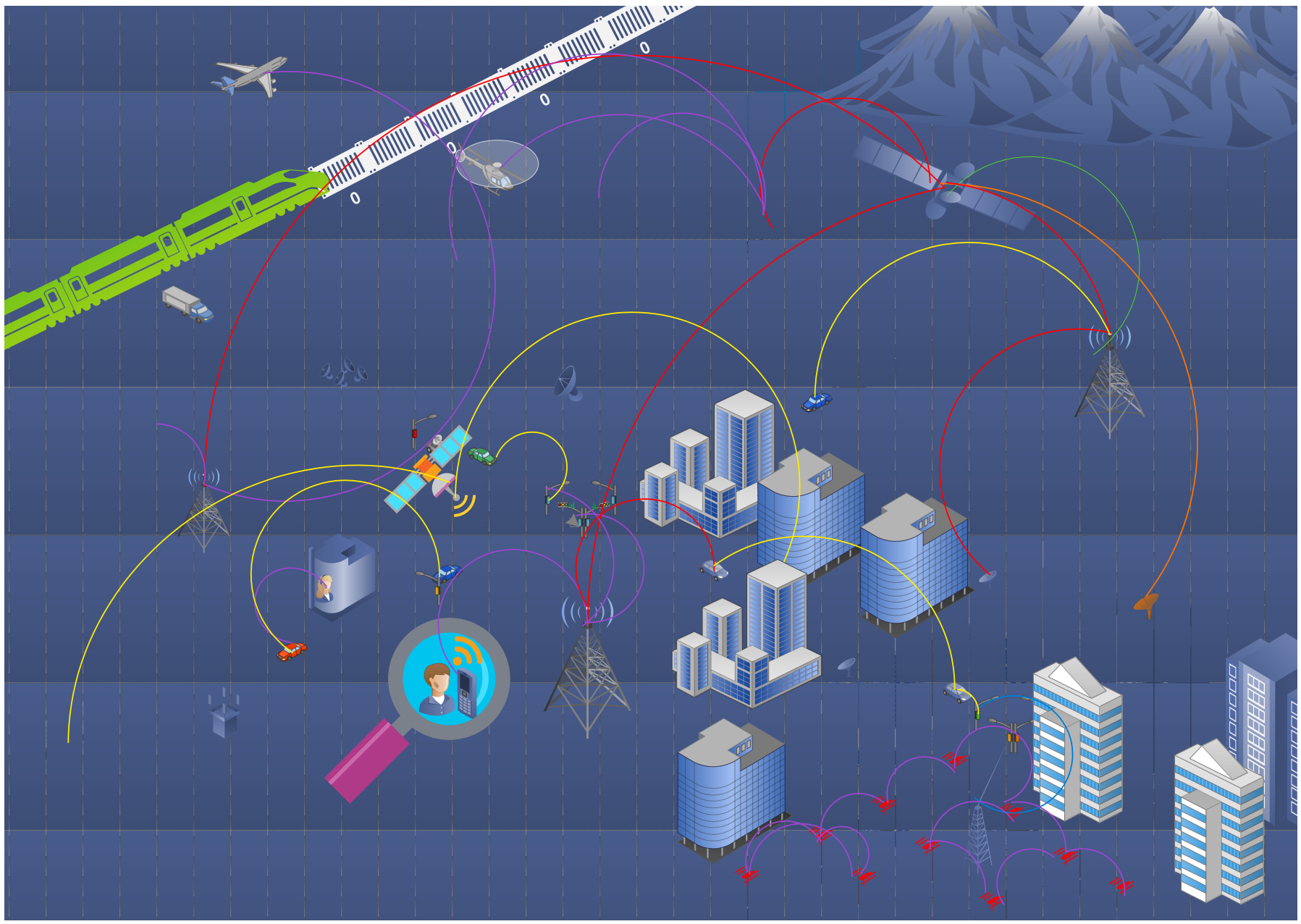

Fig. 18: An overview of the communication structure of a smart vehicle

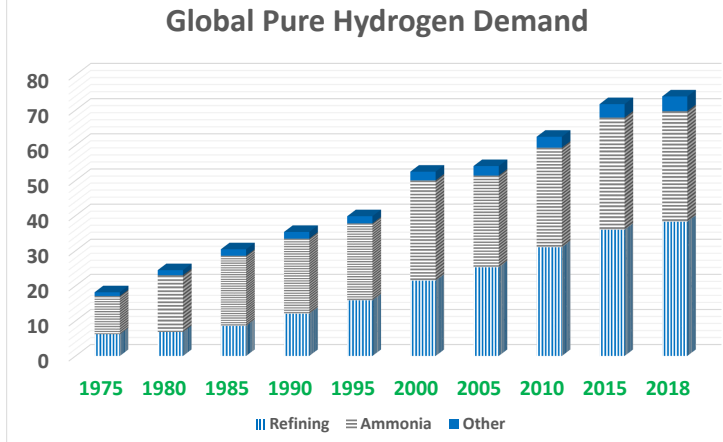

Fig. 19: Global Hydrogen Demand

smart cities results in resolutions of hurdles in flying cars and transportation infrastructure with relative ease.

\section{F. Eco-Friendly Systems}

In this subsection, the environmental-friendly systems are reviewed that are based on smart cities. Renewable energy sources play a vital role in maintaining a healthier environment. Not only this, but these resources provide effectively efficient methods to obtain energy resources. The authors in [192] reviewed research focusing on a solar photovoltaic hybrid system for eco-friendly electric vehicle charging and house based on ML system. The authors also proposed a comparative analysis between different methods based on logical advancements and optimization. Treatment of wastewater is one of the necessities of smart cities because it can cause diseases in nearby areas if it is not being treated. If it is flushed into the seawater, it can affect aquatic life. The authors in [193] proposed treating wastewater effluent by enhanced pond and wetland (EPW) system. The system is claimed to be ECO and cost-friendly since it contains two high rate algal ponds (HRAPs), two surface flow constructed wetlands (SFCWs), and a woodchip denitrification filter (WDF). Manufacturing products are processes in which several parameters are involved with a significant loss of power and material. The authors in [194] proposed an approach to optimize the product manufacturing procedure to conserve manufacturing energy. The researchers claim to save up to $26 \%$ of energy 
TABLE VII: Summary of Future Transportation Literature

\begin{tabular}{|c|c|c|}
\hline Ref. & Proposed Work & Outcome \\
\hline$[172]$ & $\begin{array}{l}\text { The study is the outcome of research on developing } \\
\text { and implementing various aspects of a system to } \\
\text { monitor the traffic and incidents intelligent enough } \\
\text { to operate independently. }\end{array}$ & $\begin{array}{l}\text { The research is focused on the smart road monitoring system using photo } \\
\text { radar that collects, processes, and analyzes big data related to incidents and } \\
\text { emergencies on the road. Data collection is done using various sensors, } \\
\text { including built-in car navigation systems, weather stations, video surveillance } \\
\text { systems, mobile communication equipment, speed cameras. }\end{array}$ \\
\hline$[171]$ & $\begin{array}{l}\text { This paper is about the cloud-based implementation } \\
\text { of the model used to improve road safety. Distributed } \\
\text { smart devices were used for the purpose by the } \\
\text { authors. }\end{array}$ & $\begin{array}{l}\text { The system proposed is based on edge devices and their interaction with cloud- } \\
\text { based servers. These devices were installed in various countries, including Italy, } \\
\text { Romania, Qatar, and United Arab Emirates, and provided positive results while } \\
\text { providing room for further improvements. }\end{array}$ \\
\hline$[180]$ & $\begin{array}{l}\text { To minimize the greenhouse effect, car manufac- } \\
\text { turers worldwide consider shifting the vehicles to } \\
\text { electric or hybrid fuel engines. Countries bring this } \\
\text { change regularized in their governing law and regu- } \\
\text { lations. }\end{array}$ & $\begin{array}{l}\text { This paper covers electric fuel cell technologies. It provides a comparison, } \\
\text { pros, and cons of the existing and proposed typologies of electric fuel cells by } \\
\text { focusing on key performance indicators such as energy efficiencies, hydrogen } \\
\text { consumption, and impact on the performance of subsystems. }\end{array}$ \\
\hline$[181]$ & $\begin{array}{l}\text { The automobile industry ranks in higher grades while } \\
\text { counting for the global emission percentage. The root } \\
\text { cause of this higher participation is fossil fuel as the } \\
\text { primary fuel for vehicles. }\end{array}$ & $\begin{array}{l}\text { This research provides a comprehensive review of currently used different kinds } \\
\text { of fuels in the automobile industry. It also highlights the advancements made } \\
\text { in the current path in this area. The paper also comprehensively covers these } \\
\text { technologies from a future perspective. }\end{array}$ \\
\hline$[182$ & $\begin{array}{l}\text { Extreme traffic, overuse, repair work, emergencies, } \\
\text { and other allied issues made road transportation } \\
\text { a hassle full mode of transportation. The research } \\
\text { focuses on the use of air transportation to study } \\
\text { challenges in getting a consumer-level adoption of } \\
\text { air-level transportation using flying cars. }\end{array}$ & $\begin{array}{l}\text { This paper presents a comprehensive study approach towards the use of flying } \\
\text { cars. The challenges of adopting standards and regulations to govern effec- } \\
\text { tive air vehicle transportation, safety, human factory, cybersecurity proofing, } \\
\text { navigation, economies of scale, and infrastructure development are discussed. }\end{array}$ \\
\hline $\mid \overline{183}$ & $\begin{array}{l}\text { Flying car transportation is in the full-scale research } \\
\text { and development phase, and it is anticipated that } \\
\text { consumer interaction with such a mode of transporta- } \\
\text { tion will start by } 2025 \text {. The urban shared transport } \\
\text { services like Uber and Lyft are expected to adopt it } \\
\text { mainly due to economies of operations. }\end{array}$ & $\begin{array}{l}\text { The paper is focused on an important and yet to explore research area of } \\
\text { the customer point of view if they are willing to accept this new mode of } \\
\text { transportation, i.e., flying cars, and the questions in their mind. The outcomes } \\
\text { show that various socio-demographic characteristics can affect customers' } \\
\text { willingness to adopt the flying mode of shared transportation. }\end{array}$ \\
\hline$|\overline{177}|$ & $\begin{array}{l}\text { Smart cities development and growth worldwide } \\
\text { bring needs for improvement and advancement in } \\
\text { multiple aspects of the city, and transportation is } \\
\text { one of them. Automation of transport infrastructure } \\
\text { is one key area that is the main focus of this paper. }\end{array}$ & $\begin{array}{l}\text { The paper's primary focus on the important components of smartness in the } \\
\text { smart city. The paper divides them into three dimensions, the smart devices as a } \\
\text { building block collectively known as smart cells, communication infrastructure } \\
\text { referred here as ICT, and development mechanism to combine two for the } \\
\text { smartness. The paper later reviews the Chinese smart cities' smart transportation } \\
\text { infrastructure as a case study. }\end{array}$ \\
\hline $\mid \overline{178}$ & $\begin{array}{l}\text { The autonomous self-driving car is the new future } \\
\text { of transportation currently in the final stages of } \\
\text { research. The key challenges in this regard are risk } \\
\text { identification and treatment while self/autonomous } \\
\text { driving. }\end{array}$ & $\begin{array}{l}\text { This study treats those self-driving risks using a risk prioritizing approach. } \\
\text { A hybrid multi-criteria decision-making (MCDM) approach is proposed for } \\
\text { risk prioritizing. After validation using fuzzy analysis, the proposed model } \\
\text { reveals that the model produces reliable results presenting the impreciseness } \\
\text { and deficiencies in the decision-making approaches. }\end{array}$ \\
\hline
\end{tabular}

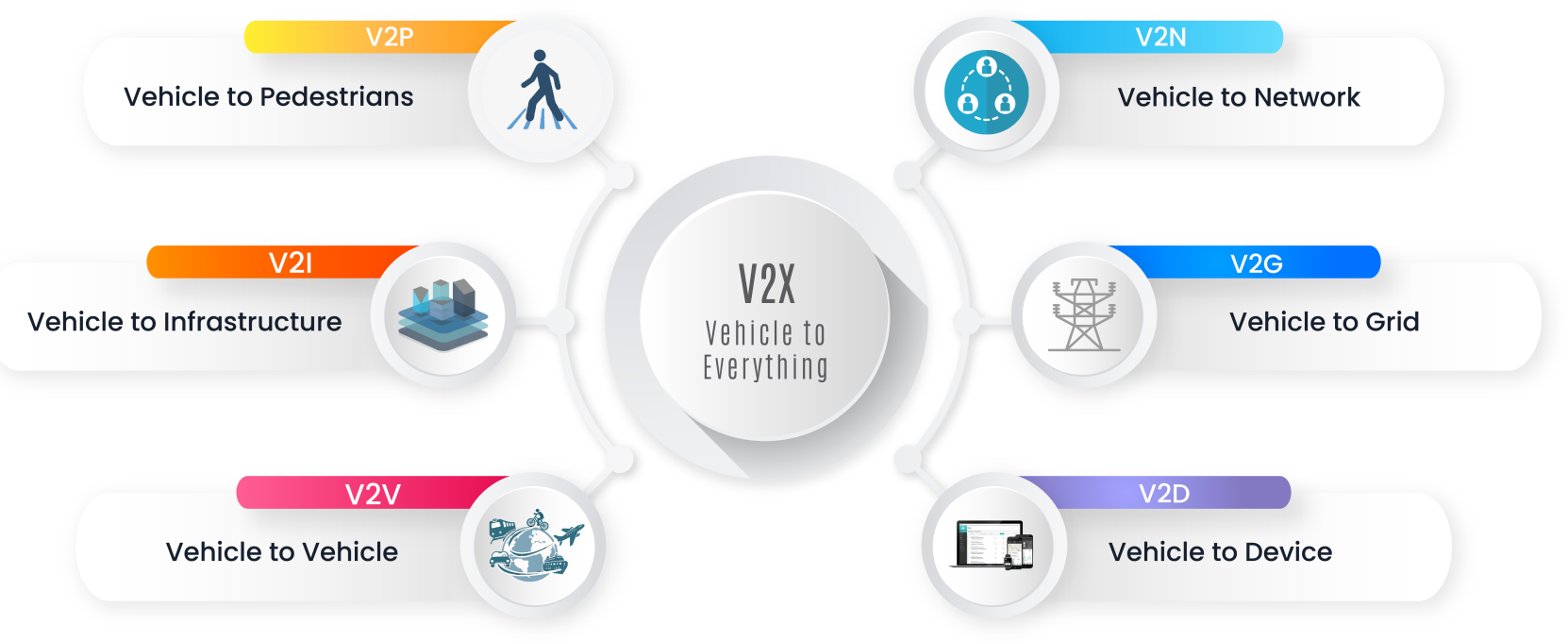

Fig. 20: Vehicle To Everything (V2X) 
consumption in the manufacturing process. The authors in [195] examined HC600a refrigerant to verify its eco-friendly characteristics, which include electric power consumption, coefficient of performance (COP), cooling load, and pulldown time (PDT). The authors in [196] proposed an approach to type-2 fuzzy several parameter methodologies to evaluate the smart city environment's waste collection systems. The researchers in [197] proposed a system to conserve the energy being consumed by the street light for the smart cities.

Critical Analysis: The future of this planet is considered brighter if and only if we modernize ourselves in a way that could be earth-friendly. The reviewed studies highlight the importance of earth-friendly technologies and propose a healthier solution to conventional non-environmental friendly technologies. The studies also perform a comparative analysis to verify the impact of green technologies and methodologies on the environment.

1) Electric Vehicles: In this subsection, electric vehicles have been reviewed in the context of smart cities. It is one of the significant components to make the environment healthier. These eco-friendly vehicles also have some parameters to be considered before being deployed in smart cities since they must be an efficient solution to the conventional ones. The authors in [198] proposed an efficient algorithm to monitor IoT-enabled electric vehicles' power consumption and make their efficiency better. The researchers in [199] proposed cybersecurity systems to make autonomous electric vehicles safer from cyber-attacks. The authors in [202] improvised an approach to help the electric vehicles locate the charging stations in smart cities. The authors in [203] proposed a thorough study over the effects of electric vehicles on smart cities. They highlighted the overall power consumption of cities and the availability of electric vehicles availability at multiple places. The authors in [204] proposed a DL approach to monitor and optimize electric vehicles' power consumption in smart cities. The authors in [205] proposed a framework to monitor the electric-vehicles driver's preferred routes and the power consumption.

Critical Analysis: The reviewed studies show that the prominent beneficial electric vehicles have some crucial parameters to be configured to be efficient in real meaning. The Internet-of-things and Edge of things enabled a new ecofriendly transport machinery era with better capabilities and efficiency. This revolutionizes the way of transport by adding an intelligent transportation system.

2) Waste Processing: Waste recycling has always been one of the major problems of modernization because it requires a good dumping area and a proficient methodology to convert the waste material into re-usable material. It also plays a major role in sustaining the healthier and good economy of a smart city. The authors in [200] surveyed the traditional methods of waste management systems and proposed multiple approaches to make the dumping and recycling procedures economical and efficient. The researcher in [201], [206] proposed an IoT empowered waste collection system. This system monitors the waste bin through the sensors and indicates the management authority to empty the waste bin when it is needed. The authors in [207] studied 3Rs (reuse, recirculation, recycling) and increased the products' life span. Their study promoted the efficient management of municipal waste in the context of circular economy vision. The authors in [208] studied the barriers between the IoT-based smart waste management system and the local policies. However, they define the necessary changes needed to optimize the IoT-based waste management system. The researchers in [209] proposed a waste management approach using Cuckoo Search Algorithm to monitor the smart waste bins. The researchers in [210] proposed an algorithm named as two-step heuristic algorithm multiple trucks routing algorithm (MITRA) for identifying the optimal ways for the waste-collecting vehicles in the smart cities. Finally, the authors in [211] highlighted the importance of waste management systems in the context of smart cities.

Critical Analysis: Waste management is a crucial task for modern cities but doing it efficiently is the art that makes it suitable for smart cities. The reviewed studies highlight the importance of waste management regarding the city's health and overall cleanliness. The reviewed research emphasizes the more competent IoT-based method to do this task. Table VIII presents the Eco-Friendly Systems Literature section in tabular form.

\section{G. Cyber Security}

To ensure the smooth operations of all smart cities' functions, the whole system's security and components are critical. The confidentiality, integrity, and availability of the functions are necessary to provide relevant services to other city's integrated system components. The significant challenges to the security of future smart cities are identifying threats/malicious activity within the systems, malfunction detection, security of the system's data and access, and its availability to its consumers (either other systems or citizens) [212].

As the smart city is composed of different aspects of city life working in a coordinated way with a technical infrastructure to support management, governance, and control of the city affairs, working on one aspect may depend on the output of another aspect's functionality. Hence the unavailability of one aspect of the smart city may disrupt city life in other dependent aspects. The systems must be designed to have a backup plan to support the functionality (even in the reduced form to keep on the only critical functions of that aspect) and maintain the business continuity.

Security is the prime part of healthcare data management and governs under strict rules like the health insurance portability and accountability act (HIPAA) in the United States. Many kinds of research were conducted to utilize blockchain in the healthcare industry for healthcare data management, data privacy, data protection, data handling, and efficiency in data processing [213]. MIT's computer science and artificial intelligence lab (CSAIL) came out with an implementable solution with the name of CryptDB for managing query processing over encrypted data [214]. The same was analyzed for its implementation in the healthcare industry with high throughput applications and was found practically implementable in dataprotected scenarios of national-level healthcare systems [215]. Ensuring privacy while sharing required data is another aspect 
TABLE VIII: Summary of Eco-Friendly Systems Literature

\begin{tabular}{|c|c|c|}
\hline Ref. & Proposed Work & Outcome \\
\hline$|194|$ & $\begin{array}{l}\text { The authors proposed a solution for saving produc- } \\
\text { tion and transportation energy. }\end{array}$ & $\begin{array}{l}\text { By optimizing the distribution of processes into machines and categorization } \\
\text { machines in manufacturing cells, we can save production and transportation } \\
\text { energy. A job shop is energy efficient replacement of a cellular manufactur- } \\
\text { ing system optimally formed. During cellular manufacturing method without } \\
\text { affecting workload, almost } 26 \% \text { of energy is saved. }\end{array}$ \\
\hline$|195|$ & $\begin{array}{l}\text { The authors proposed a novel technique for enhanc- } \\
\text { ing and improving refrigeration system performance. }\end{array}$ & $\begin{array}{l}\text { To enhance and improve the performance of refrigeration systems, performance } \\
\text { characteristics were investigated through experiments and replace HC600a with } \\
\text { HFC134a in eco-friendly refrigerants. The experiments' results prove that when } \\
\text { using } 46 \mathrm{~g} \text { of hydrocarbon, COP increased by } 32.2 \% \text { with an energy reduction } \\
\text { of } 4.5 \% \text { in the refrigerant. }\end{array}$ \\
\hline$|\overline{198}|$ & $\begin{array}{l}\text { The authors confer the importance and need of } \\
\text { electric vehicles using IoT technology, which helped } \\
\text { monitor the electric vehicles' battery life. }\end{array}$ & $\begin{array}{l}\text { Using IoT technology, the efficiency of the proposed technique and tested the } \\
\text { proposed technique by performance results and visual analysis method on the } \\
\text { vehicle is improved. It observed that the implementation cost is lesser where } \\
\text { the vehicle's capacity is almost increased by } 74.3 \% \text { after monitoring through } \\
\text { sensors. }\end{array}$ \\
\hline$[\overline{199}]$ & $\begin{array}{l}\text { Proposed how to achieve high-performance cloud } \\
\text { services, assorted, and solve security problems re- } \\
\text { garding communication during deployment of ad- } \\
\text { vanced technologies in smart cities. }\end{array}$ & $\begin{array}{l}\text { The authors proposed an automated secure cloud service availability method } \\
\text { for the smart vehicles included an intrusion detection system (IDS) for other } \\
\text { security attack detection and different services for users requirements, quality } \\
\text { of experience, and quality of service. }\end{array}$ \\
\hline$[200$ & $\begin{array}{l}\text { The authors presented a novel IoT application for } \\
\text { waste transportation and illustrate a case study design } \\
\text { for transportation processes of smart waste manage- } \\
\text { ment for Islamabad city. }\end{array}$ & $\begin{array}{l}\text { The authors presented an IoT-based smart waste transportation solution. The } \\
\text { system was simulated and modeled at the city level to prove the proposed } \\
\text { solution's effectiveness (performance gain and resource-saving). }\end{array}$ \\
\hline 201 & $\begin{array}{l}\text { The authors reviewed and investigated many smart } \\
\text { city issues directly connected with increased waste } \\
\text { material and difficulty to handle. }\end{array}$ & $\begin{array}{l}\text { This paper proposed a municipal solid waste management system (MSWM) } \\
\text { and smart waste bin monitoring (SWBM) for smart waste management based } \\
\text { on IoT technology. The proposed smart waste management system solves waste } \\
\text { material problems and provides IoT-based automatic waste collection in smart } \\
\text { cities. }\end{array}$ \\
\hline
\end{tabular}

that requires greater concern. Sharing of required information with authenticity while ensuring privacy protection is the theme discussed in the [216] with the provision of a complete framework based on blockchain in order to share information of the individual with the Government, companies, and other stakeholders with privacy preserved surety and complete authenticity.

As various aspects of smart cities have different financial attractions to the malicious actors, hence these actors try to find a loophole or vulnerability. It may gain success for the malicious actors, Due to the multitude of technologies used in developing different aspects and their possible incompatibilities or misconfiguration. On the other hand, cybersecurity defenders tried their best to patch all potential vulnerabilities and close all possible loopholes to stay ahead of the hackers [217].

Ensuring confidentiality, integrity, and data availability are of utmost importance in the future smart city. Hack proofing of the system must be guaranteed that processes are self securityaware and must have an inbuilt resistance to any unauthorized and malicious attempt to interact with them or their data.

1) Digital Forensics: Crime existence is expected to the same extent in smart cities as they are in traditional cities. To tackle the crime and catch the culprit, forensics can help the investigators reach a conclusion and get their hands on the facts and evidence that can be used to convict the criminals. Keeping in view the sizeable technological base in any smart city aspects, there is a significant chance that crime might involve digital aspects in their commitment [218]. To investigate such crimes, a solid digital forensic base must be established to investigate the future crime committed. However, to do so, maintenance of balance carefully between data/log collection and breach of individual/organization's privacy is a challenge [219]. A study [220] covers the forensics investigation aspects of increased usage of social media applications by criminals for communication purposes which is usual trends nowadays, keeping in view the confidentiality and integrity provided through end-to-end encryption.

Secure management of evidence throughout its life cycle is also critical. After becoming the prospective evidence, secure handling, processing, and digital evidence storage are crucial to ensure the integrity aspect. A blockchain-based solution "LEChain" [221] is one of the current developments in this regard. Further researches are underway to target various aspects of digital forensics.Figure 21 presents the life cycle of digital forensics.

The process of digital forensics starts with crime reporting. It ends after the completion of the case passing through the phases of collection of artifacts, their examination for possible linkages with the crime, analysis of suspected artifacts for data collection, reporting of findings in a presentable court way, testimony, and presentation of evidence during the trial and completion and storage of the evidence after the conclusion of the case. Keeping the evidence's integrity and keeping the chain of custody document against each artifact is of the utmost importance during the whole process. Different kinds of systems, sources, and devices used in different scenarios and processes to fulfill tasks in different smart cities' systems make this harder to have standardized data collection procedures [222].

Another challenge in digital forensics is the availability of expert digital forensics practitioners in sufficient numbers. 


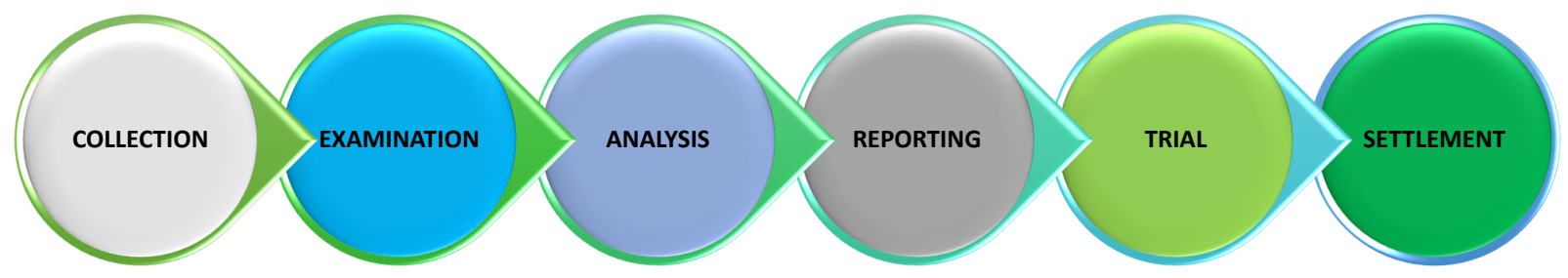

Fig. 21: Digital Forensics Lifecycle

Further, due to continuous advancements in the technology arena, the experts must update themselves about the recent advancements to remain familiar with the current state of technology to analyze it as and when required [223].

A coordinated approach is always a better approach. A centralized pool of experts may be managed where the expertise is shared to bring all of them aware of the latest trends in technologies. It provides expert-level forensics to digital crimes and has a platform for experienced value addition and knowledge addition and development. A central platform was later transformed to provide digital forensics as a service (DaaS) to other organizations like law enforcement agencies. Such DFaaS offerings are beneficial both in costeffectiveness, knowledge base development, expert operations, skills pooling, enhancement, and legal acceptability [224].

Extensive usage of IoT devices and their intentional or unintentional involvement in cyber crimes shifts the focus of researchers in the forensic field to the forensics of IoT devices to investigate ever-increasing cybercrimes. The forensics of IoT has further encompassed forensics of Cloud, Network, and IoT devices themselves. The mind map presented in figure 6 of [225] is the best graphical representation of the challenges faced in IoT forensics by current researchers and practitioners. Critical Analysis: The use of state-of-the-art technologies, automation, and digitization in future smart cities with big data management and analysis and informed decision-making will eventually result in crime too shifted from traditional domains to the digital domain. To safeguard the processes, people, and technology infrastructure of the future smart city, effective investigations in criminal cases will eventually require enhanced logging and monitoring. The same data will then used for digital forensics investigations. Having the required number of experts, having expertise in various technical domains of smart cities, and in-depth analysis capability development depends on human resource development in cybersecurity and digital forensics. Therefore, it is anticipated that workforce development and technical research center establishment must be planned for current and future smart cities to fulfilling the everyday need of cyber savers, preventing and investigating digital crimes in future smart cities.

2) Intrusion Detection Systems: The increasing connectivity in smart cities also results in a growing threat to every connected node. The enhancement of the cybersecurity side is a crucial challenge in the smart cities' future. Various security threat detection techniques are evolved to counter the threats in smart cities' connected world, including analysis of protocol, analysis of traffic, analysis of behavior, analysis of control process, and mining of data [230]. In this regard, intrusion detection systems remedy ongoing events within the system to look for abnormal behavior of already known malicious activity patterns. In case of detection, the same will be intimated through alerts and other configured measures to contaminate the hazards [226], [227]. Yearlong researches in past results in very effective and efficient techniques for the detection of such event; however, this area requires ongoing research and development not only to fulfill the need for improvements in already flourished techniques but the significant chunk of efforts are devoted to the detection of new threats and pattern identified regularly. The intrusion is detected usually based on three aspects which are

- The anomaly detection

- The specification detection

- The misuse detection

However, current research is more focused on detecting the intrusion in advance before the incident [231]. Incidents can cause serious trouble and may disturb the everyday life of citizens, along with other losses. Hence early detection is key to uninterrupted smooth life within the cities, traditional or smart.

Critical Analysis: An intrusion detection system (IDS) is undoubtedly the critical component of todays and future datadriven smart cities. Due to the critical nature, many systems rely on correct data availability at the correct time without irrelevant processes/people to have their hands on the data. To let all functions of the smart cities work flawlessly, detection of flaws before their occurrences can only be done using a proactive approach, and the best practice is the use of IDS. However, the attacker always has a universal advantage to attack that device on the target network, which has the least security. IDS can help us in such situations by the pattern of traffic matching from the expected attacks.

3) Intrusion Prevention Systems: An intrusion prevention system is that component of the cybersecurity domain that comes into action after successfully detecting the system's cyberattack. Intrusion prevention systems defend the system 
TABLE IX: Summary of Cyber Security Literature

\begin{tabular}{|c|c|c|}
\hline Ref. & Proposed Work & Outcome \\
\hline 213 & $\begin{array}{l}\text { This study presents a systematic literature review } \\
\text { (SLR) of } 42 \text { articles on the subject, presenting state- } \\
\text { of-the-art research in blockchain usage for health- } \\
\text { care. }\end{array}$ & $\begin{array}{l}\text { The article gives way out to research and implement blockchain in health- } \\
\text { care like remote healthcare facilitation, medical diagnostics, fraud detection, } \\
\text { avoidance, legal and regulatory compliances, etc. }\end{array}$ \\
\hline 217 & $\begin{array}{l}\text { The future smart cities have an attraction for ma- } \\
\text { licious actors both at the state level or otherwise. } \\
\text { The paper categorizes the vulnerabilities and risks } \\
\text { to smart city technologies in five categories. }\end{array}$ & $\begin{array}{l}\text { Authors emphasized the widening of existing tools and technologies used to } \\
\text { mitigate attacks on the technical infrastructure of smart cities by adding / } \\
\text { inclusion of security at the design time. Further to this widening effort, depth } \\
\text { of security can be gained by doing mandatory regular vulnerability assessment, } \\
\text { patch management, regulations, and sops, and the establishment of CERT for } \\
\text { incident response. }\end{array}$ \\
\hline$\overline{222} \mid$ & $\begin{array}{l}\text { Technology everywhere increases the risk of expo- } \\
\text { sure and compromise of systems as well as their } \\
\text { information. The book focuses on the outcome of } \\
\text { the EU COST project CRYPTACUS. }\end{array}$ & $\begin{array}{l}\text { CRYPTACUS focuses on the latest researches and development to address } \\
\text { scientific challenges in the domains of information security, information } \\
\text { privacy, cryptography, and embedded systems. The article is mainly focused } \\
\text { on Ubiquitous Devices, Lightweight Cryptography, Contemporary Symmetric } \\
\text { Encryption, Block cipher standardization, and Electronic Identity Technologies. }\end{array}$ \\
\hline$\overline{223}$ & $\begin{array}{l}\text { Extended use of technologies in everyday life also } \\
\text { increases the risk of cybercrime linked specifically } \\
\text { with data theft, exposure, or leakage. This then } \\
\text { increases the need for the availability of Expert } \\
\text { Forensic Practitioners. }\end{array}$ & $\begin{array}{l}\text { The focus of this research is to evaluate and identify the various skills } \\
\text { that are required for cyber forensic professionals and practitioners in today's } \\
\text { technology-based world. The multi-agent approach is evaluated for the Iden- } \\
\text { tificationidentification of the skills which are effective for forensic laboratory } \\
\text { investigation. }\end{array}$ \\
\hline$\overline{\mid 226}$ & $\begin{array}{l}\text { The preferred communication mode is shifted to } \\
\text { wireless in the past decade. The openness of the } \\
\text { physical medium of wireless networks makes it lu- } \\
\text { crative for attackers. Routing attacks are also com- } \\
\text { mon in such scenarios. }\end{array}$ & $\begin{array}{l}\text { This paper evaluates LEACH protocol with levenberg-Marquardt neural net- } \\
\text { work (LEACH-LMNN) in a real-life example to evaluate the network's lifetime. } \\
\text { This paper also differentiates normal and normalized traffic by performing } \\
\text { intrusion detection using a gated mechanism (LSTM and GRU) in the same } \\
\text { wireless sensor network with a higher detection rate and lower false-positive } \\
\text { ratio. }\end{array}$ \\
\hline$\overline{\mid 227} \mid$ & $\begin{array}{l}\text { Smartphones have become a common commodity for } \\
\text { everyone. The Digital and technological aspect of } \\
\text { smartphones becomes an integral part of our daily } \\
\text { life. The associated risks due to inherent vulnerabil- } \\
\text { ities also did the same. }\end{array}$ & $\begin{array}{l}\text { The authors proposed two layers approach named PARCIV to recognize the } \\
\text { physical activities done by the owner correctly. For this purpose, an android } \\
\text { based application was developed to collect labeled data from the end-users } \\
\text { using phone sensors. PARCIV achieved } 99 \text { percent accuracy on the self- } \\
\text { collected data set, whereas } 95 \text { percent accuracy on the publicly available data } \\
\text { set. }\end{array}$ \\
\hline$|\overline{228}|$ & $\begin{array}{l}\text { In the current era of post 4IR, the industrial founda- } \\
\text { tion is now widely based on IoT devices. The mali- } \\
\text { cious activity on such devices will have a devastating } \\
\text { impact as they are no more network-only devices and } \\
\text { interact with real-world systems. }\end{array}$ & $\begin{array}{l}\text { This paper proposed a novel method for smart cities using a DL algorithm that } \\
\text { results in intrusion detection in a relatively shorter time with high detection } \\
\text { accuracy. }\end{array}$ \\
\hline [229] & $\begin{array}{l}\text { As different layers of smart cities are interdependent } \\
\text { and are closely mixed, risk manageable to one layer } \\
\text { might be destructive for the other layer. Security of } \\
\text { these intermixed layers is a crucial challenge. }\end{array}$ & $\begin{array}{l}\text { This study provides a comprehensive insight into the technical, economic, } \\
\text { social, and political challenges the smart city faces. Some areas covered in } \\
\text { the paper are privacy and security, mobile and smart devices, healthcare, com- } \\
\text { munication, power, infrastructure, citizen's life, and governance. The authors } \\
\text { also offered vital directions for future research. }\end{array}$ \\
\hline
\end{tabular}

from being harmed from cyberattacks according to the administrator's pre-defined set of rules. With the modernization of traditional cities into IoT-powered smart cities, there arises a much need for intrusion prevention systems to secure the smart cities [232]-[235]. The authors in [228], [236], [237] proposed a DL based approach to detect and prevent cyberattacks over smart cities. The proposed system also consists of an IoT feature extraction methodology to enhance the proposed model's accuracy. The authors in [238] review the current state-ofthe-art security mechanism for the e-government applications and assess their capabilities. Finally, they also proposed an $\mathrm{AI}$ and blockchain technology-based decentralized mechanism to provide all-in-one security in the context of smart cities. The authors in [239] proposed intrusion detection algorithms for the smart cities' wireless communications. The proposed algorithms are based on threshold-based intrusion detection systems (TBIDS) and multi-path-based intrusion detection systems (MBIDS). A cross-layer methodology has been integrated into the application layer and network layer for intrusion detection. The authors in [147] proposed a DL based approach for the detection of botnet attacks over IoT-based smart city components. The proposed approach mainly analyzes the DNS data to identify and prevent the system from attack. The authors in [240] proposed a multilevel intrusion prevention system that includes component level, system level, and security operation center for the smart cities. The authors in [241] proposed a proficient algorithm-based intrusion prevention system that mainly detects DDOS attacks over the smart city applications. The proposed system analyzes the network bandwidth and data to identify the DDoS attack over the network. The authors in [242] proposed a novel approach to detect HELLO-Flood attack, Version number attack, Sinkhole attack, and Blackhole attack in the networks of smart cities. The authors in [229] thoroughly reviewed the security systems of modern technologies for smart cities.

IIoT becomes part of everyday used tools and equipment to add efficiency and control to their usage. To prevent misuse of this vast number of IoT devices, authors in [243] comprehensively cover the need, requirements, design, and implementation of the network-based intrusion detection system (NIDS) for IoT devices. The IoT NIDS ecosystem is introduced to focus on learning terminologies and state-of-theart solutions with careful evaluation of their pros and cons. In order to prevent intrusion in IoT devices, [244] analyzed and 
proposed the authentication scheme to prevent unauthenticated access to prevent the intruder having his / her entry into the network to act maliciously.

\section{Critical Analysis:}

Intrusion prevention system is one of the core components of smart cities sustainability as it ensures a streamlined working of the IoT based on technologies. If there are no IPS in the technologies, this leaves them vulnerable to cyberattacks, and thus when they get exploited, it could lead to devastation. Table IX presents the Cyber Security Literature section tabularly.

\section{H. Smart Life}

All the processes and management structures in the smart cities have a common target to achieve, and that target is to import the quality of life of that city. The increased digitization and automation bring so many changes in citizens' everyday life routines of citizens [245]. Sustainable growth is required economically - technologically, environmentally, and spatially [246]. A study [247] revealed that decreased quality of life could be turned up by giving more priority to investment in development and support of community services and social capital [247].

1) Smart Homes: To provide quality of life to its citizen, smart homes in the smart city play a backbone role. Urbanization is increasing worldwide. This exerts pressure on the cities' population, which ultimately degraded the city's life due to overpopulation. The main unit of city life is home, and technological advancement in smart home technologies gives an up-shift to live a healthy life at home [248]. Home automation is the essential aspect of smart homes, and IoT devices act as a backbone to home automation [249]. The integrated system consists of these devices must be energy conscious and have to provide a user-friendly environment to the end user [255].

Inside the home, the provision of ease by automation of everyday tasks is made using AI, IoT and IoNT devices connected with either each other directly or through central smart home manager like Alexa and google home. Such smart home manager results in standardization and compatibility of various communication and data sharing protocols as well as for the control management [256].

The life of future smart city residents will be free from unproductive tasks. An example can be quoted when the smart citizens must not care about how many eggs are there in the smart fridge of their home. The sensors in the smart fridge will keep track of the remaining inventory of the eggs in the egg basket of the fridge. Upon reaching thresh hold level, the smart fridge automatically orders the eggs from an online store. The payment was made through the citizen's credit card, and the next day, the eggs will be delivered to replenish the egg inventory. Another critical area of research in this field is activity identification within a smart city. Using the sensor, key features were extracted from the events happening within smart homes, and based upon the perceived information and rules, activities such as movement, walking, eating, bathing are identified to make smart home situation-aware [257].

Critical Analysis: The smart home's target is to provide residents with a user-friendly automated environment customized based on the residents' requirements. The Voice user interface (VOI) is heavily utilized for controlling home automation. Many big names like Google, Amazon, and Apple are trying to win the future smart homes controlled by the Assistant Devices. However, lack of standardization and interoperability are the major hurdles in this regard. Future smart cities will benefit from the advancements and improvements in these technologies.

2) Smart Healthcare: Advancement in technology in the healthcare sector is revolutionary and results in making humans' lives secure from diseases up to a great extent. Health monitoring plays a significant role in identifying the issue at earlier stages. The use of AI, ML, and IoT devices in smart homes together provides healthcare monitoring at the personal level virtually 24/7, As emphasized in [250]. Availability of high-speed communication networks, cloud computing, and multimedia services in the smart city also possess enormous potential in the field of telemedicine, remote medical services, and medical data analysis and movement [251]. Figure 22 presents the components of the personal healthcare monitoring system.

Healthcare data generated from multiple types of devices produced from different vendors have different accuracy and storage interfaces. In case their accuracy can be made above specific threshold w.r.t medical standards. It can be integrated with the medical electronic health record of the person so that his / her general medical conditions/vitals may be available daily for later analysis by the healthcare professional as per requirement [252]. The building blocks for such a system are composed of the device itself, the patient/user, connectivity, gateways, cloud-based servers, and analytical engine [253]. The output from the analyzed and processed results can then be accessed centrally by the healthcare professional as and when required.

Critical Analysis: Healthcare facilities are a necessary part of the city's life to take care of the citizens' wellbeing. Technological advancement in the smart cities also significantly increase the possible ways the health of the citizen of such cities can be taken care of. Network and compute facilities emerge H-IoT as a new industry by creating a demand for personal health assistants and their continuous data management, storage, and analysis regarding one's health dynamics [258], [259].

3) Smart Citizen: The whole concept of a smart city revolves around the citizens [260]. They are the central element of smart cities. All other functions of smart cities are meant to facilitate the city's smart citizens in one way or another. The smart city is backed by creativity and new ideas, and the source of these ideas and creativity are its citizens. Empowerment of the smart city citizens by their inclusion or participation within the city can be measured to identify the bond between citizens and the city [261]. A major change was observed when the funding programs for creating smart cities by the European Commission were renamed as European innovation partnerships for smart cities and communities (EIP-SSC). They have managed an entire cluster mainly focused on citizens. Hence, the reason for the latest technology integration is to produce innovative solutions which can provide quality of 
TABLE X: Summary of Smart Life Literature

\begin{tabular}{|c|c|c|}
\hline Ref. & Proposed Work & Outcome \\
\hline 246 & $\begin{array}{l}\text { The authors review assistances in occupant's sustain- } \\
\text { able living in communities and buildings, highlight } \\
\text { and discuss issues related to sustainable development } \\
\text { of the environment, and emphasize the lives from } \\
\text { green and smart design perspectives. }\end{array}$ & $\begin{array}{l}\text { The authors provide a detailed survey on the models, proposed methods, } \\
\text { and available applications related to sustainability. The authors provide and } \\
\text { discuss the understanding and the adoption of sustainability and further research } \\
\text { challenges to sustainable communities and buildings, which will provide a } \\
\text { sustainable livelihood for the whole society. }\end{array}$ \\
\hline$\overline{248}$ & $\begin{array}{l}\text { In this study, the authors discuss smart homes and } \\
\text { smart cities and show how smart cities' performance } \\
\text { be increased and how smart homes contribute to } \\
\text { smart cities' performance. }\end{array}$ & $\begin{array}{l}\text { Based on the available literature, the authors created a model for smart cities. } \\
\text { The developed model help in two new factors of smart cities: the importance } \\
\text { of education and people in smart cities. }\end{array}$ \\
\hline$\overline{249}$ & $\begin{array}{l}\text { The authors proposed how AI techniques help and } \\
\text { use in the energy sector. }\end{array}$ & $\begin{array}{l}\text { The study explored that how AI techniques work using traditional models } \\
\text { in smart grid, robotics, IoT, controllability, cyber attack deterrence, big data } \\
\text { management, energy efficiency optimization, computational effectiveness, and } \\
\text { predictive control. }\end{array}$ \\
\hline$|\overline{250}|$ & $\begin{array}{l}\text { The research work provides a complete guide on how } \\
\text { the available sensor technology help in smart home } \\
\text { monitoring. }\end{array}$ & $\begin{array}{l}\text { The authors conducted a detailed literature review and summarized the existing } \\
\text { state-of-the-art research on available sensor technology for health monitoring } \\
\text { in the home. The authors developed four terminology monitoring functions, } \\
\text { data, unobtrusive sensor, and location for the structured analysis. }\end{array}$ \\
\hline 251 & $\begin{array}{l}\text { The authors proposed a model to improved the } \\
\text { utilization and delivery of health care resources in } \\
\text { a smart environment. This paper introduces a model } \\
\text { that can provide improved delivery and utilization of } \\
\text { resources. }\end{array}$ & $\begin{array}{l}\text { The authors developed a quality reward model to react and study the content- } \\
\text { ment factors of the healthcare systems in a smart environment. The authors } \\
\text { also proposed an algorithm called the Maximum Reward Algorithm to deliver } \\
\text { and use healthcare resources. The algorithms tested through simulations and } \\
\text { experiments, the result provides efficiency reliability and achieves } 50 \text { to } 77 \% \\
\text { performance. }\end{array}$ \\
\hline$\overline{252}\rceil$ & $\begin{array}{l}\text { To help doctors regarding patient health, for regional } \\
\text { medical unions, healthcare data analysis system is } \\
\text { proposed to assess the patient's health condition } \\
\text { based on overall data view from hospital to doctors } \\
\text { from different hospitals. }\end{array}$ & $\begin{array}{l}\text { Labels are produced from social data to search the topics related to people } \\
\text { corporeal in the joint region. Human behavior is excavated from vital physiolog- } \\
\text { ical values. Experiments described the probability of the system in supporting } \\
\text { human healthcare data examination. }\end{array}$ \\
\hline$|\overline{253}|$ & $\begin{array}{l}\text { The authors presented the available software frame- } \\
\text { work related to IoT systems in smart agriculture, } \\
\text { smart healthcare, and smart cities. }\end{array}$ & $\begin{array}{l}\text { In this paper, the authors analyze available different types of IoT architecture } \\
\text { like cloud-based, service-oriented, and layered architecture applications in } \\
\text { various areas of IoT. }\end{array}$ \\
\hline $\mid \overline{254}$ & $\begin{array}{l}\text { The purpose of this study is to identify the key } \\
\text { indicators related to citizen-centric for smart cities } \\
\text { from the participative domination practices and in- } \\
\text { habitants' accountabilities perspectives. }\end{array}$ & $\begin{array}{l}\text { This research study elaborates a systematic literature review (SLR) of social } \\
\text { enclosure pointers for structure citizen-centric in the smart cities environment } \\
\text { to achieve the proposed research aim. }\end{array}$ \\
\hline
\end{tabular}

life and easiness to the citizens [262]. For this, the citizens must be part of the city governance. In the absence of normal citizens' participation both on the responsibility fulfillment and decision-making side, the citizen-centric Government is not possible [254]. Figure 23 presents the governance of smart citizen-centric.

In a proper citizen-centric smart city, the interacting factors are government role, technological base, citizen intelligence, and cognitive liabilities [263]. It is anticipated that the future smart cities will prevail the citizen-centric approach for governance and management. In [264], the authors implemented the citizen-centric approach towards smart cities in Taiwan and based their study on 35 city services from 07 domains grouped into two domains. The results show a positive trend towards adopting the approach to improve citizens' well-being and quality of life.

Critical Analysis: The traditional city structure revolves around the city government, which was the dominating factor for its operations. Policies were devised and implemented for the city's smooth operations, and citizens have to abide by them. However, in developed countries and now in smart cities, the focus has been shifted to the citizen-centric approach. Now the systems and processes are designed in such a way to facilitate the citizen more than before. It brings the concept of participatory governance, where citizens have an active role in designing and managing governing processes. This phenomenon is extracted from the concept of democracy, which is considered the Government of the people and the people. Provision and facilitation of quality and well-being are of the utmost importance in such consideration [265]. Table $\mathrm{X}$ presents the Smart Life Literature section tabularly.

\section{Smart Economy}

Great democratization of information communication technology (ICT) around different world countries leads to a debate on resource-conserving, sustainable, smart cities, and resilient smart cities' economic development appropriate to different countries. Different continents have their own emerging patterns for urbanization, requiring strategies, policies, and diverse approaches. Each city of the country or continent will possess its challenges regarding the economic development of smart cities [260].

[266] has proposed a Unique composition of validation of sectoral improvement directions in a SMART economy dependent on recognizable proof of gaps among current and required degrees of sectoral advancement and the use of a complex of 


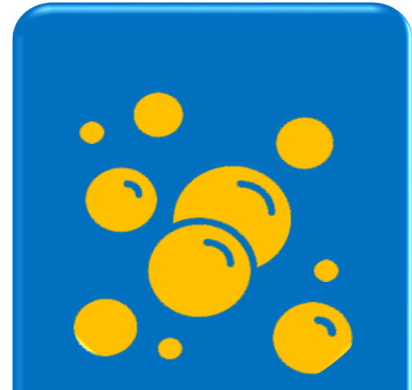

Physiological

Monitoring

Functional

Monitoring

Emergency

Detection

Safety Monitoring

Social Interaction

Monitoring

Security Monitoring

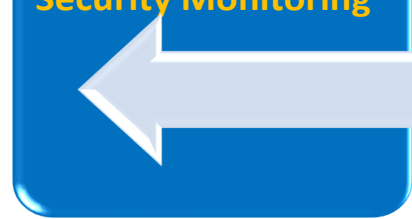

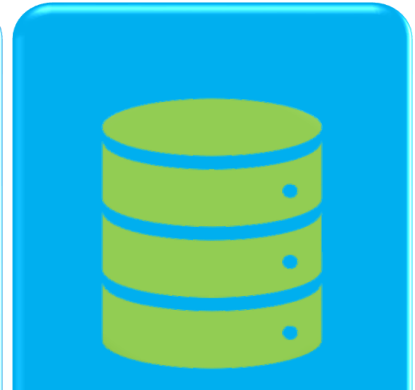

Physiology Data

Behaviour Data

Environment Data

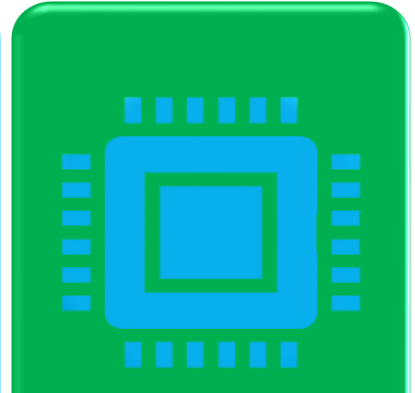

Mechanical Sensors

Electro-Magnetic

Sensors

Optical Sensors

Air Quality Sensors

Acoustics Sensors

IT / Computer

Related Sensors

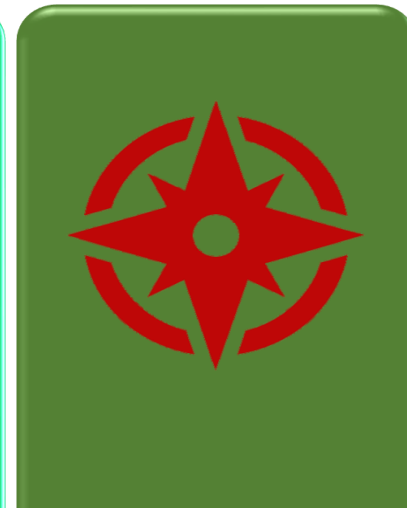

Appliance Location

End User Location

Physical Location

Emergency Services

Location

Fig. 22: Personal Healthcare Monitoring Components

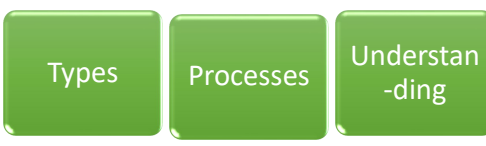

Participatory Governance
Roles of Citizens

Responsibility Ownership

\section{Citizen Centric Smart City}

Fig. 23: Citizen Centric Smart Governance [faisal]

changeable plans of action as an instrument conquering these gaps. [266] Also identified three types (horizontal, vertical, and mixed) of basic trajectories for sectoral advancement based on different business models, government support level, and available resources had been point identified. Deloitte presents the following key points that differentiate a smart city in his research article, [267]: -

- Economic competitiveness

- Quality of life

\section{- Sustainability}

showing that economic activities are the key component of a smart city. To get fruitful results, it is necessary to adopt the mitigating strategies to remove/minimize the barriers impacting industrial growth, which ultimately results in economic growth [268]. This section will undergo the three primary aspects of the economy, i.e., businesses, financial sector, and production facilities which together make an economic horizon.

1) Smart Business: Business activities are crucial to city life. A technologically coherent business system is required for future smart cities based on calculated and predicted KPIs to provide the economic and requirement-oriented system governing the need to consume the product's life cycle. The smart manufacturing system is way more productive and efficient than the traditional manufacturing approach and is incorporated by the top manufacturers by incorporating it into their production methodologies. This approach's suitability is far better in terms of capital expenditures and supply chain and allied processes along with alone manufacturing [269]. Keeping in view the limited budget available for SME's, the incorporation of advanced manufacturing technologies, especially $3 \mathrm{~d}$ printing, augmented reality, IoT, big data analytic, cloud, fog and edge computing, additive manufacturing, 
simulations, and horizontal and vertical integration, brings exponential growth opportunities to the businesses in todays and upcoming smart business world. According to [270], the major factors required to win customers' trust for the digital offering of the products are attitude, behavior, environment, and experience. Along with this, the key to success for smart business lies in the adoption of complex and dynamic strategies in a technology-based organizational environment [271]. Combining the technology with the generic processes to get more fruitful results is the target to achieve by doing the business more smartly and the core component of future smart cities.

Critical Analysis: Ways to do business are changing worldwide. Especially in the post-Covid19 world, the lockdowns and interaction less environment due to safety and care results in a boost in the business incorporating or shifting to online business models even in the traditional business world. However, in the world of smart cities, smart businesses are not only online but automated. Consider a smart fridge getting a continuous check on several eggs in the egg basket using smart sensors. Upon detecting the reaching threshold level, e.g., four eggs, automatically put the order to the nearest online store and pay through the credit card of the house owner. It also delivered on the same day using smart delivery without the involvement of the house owner to get worried about such aspects.

2) Smart FinTech: The documented economy provides blood to the financial sector of the economy. This prevents misuse of money and violation of laws. It also helps in the revenue estimation and collection for the smooth running of governance and development projects. The banking industry and other fintech organizations provide this service to the Government, organizations, and general public. The inclusion of the latest trends and technologies is on the rise in this sector compared to other smart cities [276]. The provision of smart services to the residents is the basic philosophy of smart life in a smart city, and financial services are the basic unit of this philosophy. Smart citizens, smart governance, smart business, and smart life are all significant subscribers of these services. The services also come in handy after introducing mobile banking services as now everything in the financial arena can be managed from the person's palm. However, in the future smart cities, the reliance on financial services is majorly based on AIDS (artificial intelligence and data Sciences). However, it covers broadly classical AI, modern AI, and data sciences. The classical AI includes logic, planning, modeling, simulation, image processing, decision making, and decision support system (DSS), autonomous systems, knowledge representation multi-agent systems, expert system (ES), pattern recognition, and natural language processing (NLP). Modern AI and Data Sciences include complexity science, mathematical modeling, statistical modeling, knowledge discovery, representation learning, ML, optimization, data analytic, computational intelligence, event and behavior analysis, social media/network analysis, DL, and cognitive computing [272]. FinTech companies started to utilize the power of blockchain and smart contracts to bring transparency in the processes [273].

Critical Analysis: The smart financial technology (FinTech) for future smart cities will cover the inclusion of AI, ML, and Data Analytics for the operations in the field of smart banking, smart insurance, smart lending, smart marketing, smart payment, smart regulations, smart financial risks management, smart financial security, smart trading, and smart wealth management. These inclusions will result in a scalable, expandable, auditable, and available financial management platform to deliver $\mathrm{B} 2 \mathrm{~B}, \mathrm{~B} 2 \mathrm{C}, \mathrm{C} 2 \mathrm{C}, \mathrm{G} 2 \mathrm{~B}, \mathrm{G} 2 \mathrm{C}$, and $\mathrm{G} 2 \mathrm{G}$ services most efficient and auditable way.

3) Smart Factories: The production process is the part that shows the industrial strength of the country/city. Realizing the importance of manufacturing, many cities started offering special industrial zones with all the facilities for commercial manufacturing ventures or molding the industrial sector of the city to become a hub for expert and quality manufacturing for specific technology areas/products. Moreover, the research is now going to multi-plant smart manufacturing. Multiple parties with expertise in their particular areas now work together to produce final products using smart manufacturing. A recent research [277] proposed a detailed review of identity and authorization management frameworks for Industrial IoT (IIoT), which is the critical requirement for secure smart manufacturing.

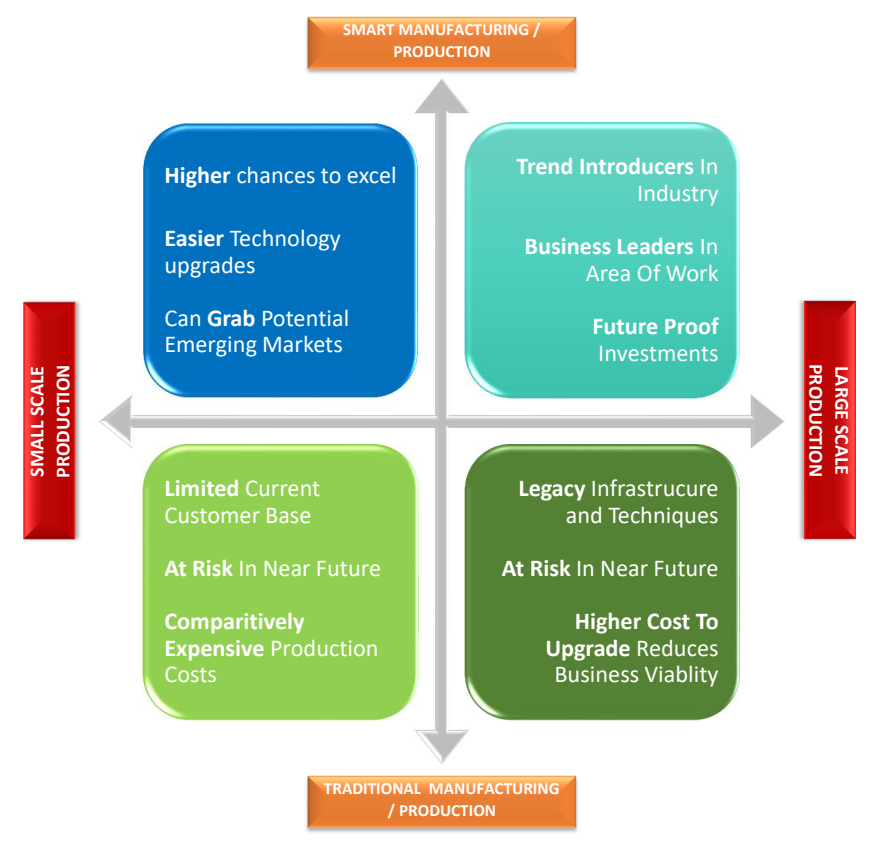

Fig. 24: Prospective Benefits/Threats To Smart Production

To have automated control, the following three major areas of manufacturing must be shifted to data-based automated control [274].

- Product Tracking Technology

- Process Data Acquisition System

- Fault Detection based on Neural Networks

Based on the data gathered from all these aspects, the production quality, quantity and operations can be effectively controlled and automated. As well as reaping of further additional benefits like production trend analysis, future prediction, 
TABLE XI: Summary of Smart Economy Literature

\begin{tabular}{|c|c|c|}
\hline Ref. & Proposed Work & Outcome \\
\hline |267 & $\begin{array}{l}\text { A smart economy is considered one of the key com- } \\
\text { ponents of smart cities. Key issues and challenges to } \\
\text { the smart economy were discussed in the literature. } \\
\text { The article reviews the published literature for the } \\
\text { same. }\end{array}$ & $\begin{array}{l}\text { The article presents bibliographic coupling of the published literature on smart } \\
\text { cities and economy and specifically focused on the smart economy. The study } \\
\text { found out that a major portion of such researches was done in the USA and } \\
\text { India. The main keywords identified are smart cities, urbanization, governance, } \\
\text { innovation, and economy. }\end{array}$ \\
\hline$|266|$ & $\begin{array}{l}\text { digitization, technology development, and develop- } \\
\text { ment in IoT increase pressure of sectoral develop- } \\
\text { ment in smart economies. }\end{array}$ & $\begin{array}{l}\text { Identifying the gap between actual and required sectoral development is very } \\
\text { necessary and the theme of this research. The authors built the matrix based on } \\
\text { digitization, market trends, and the development in the relevant sector in ad- } \\
\text { vanced technologies to identify government support and resource management } \\
\text { requirements for vertical, horizontal, and mixed sector development. }\end{array}$ \\
\hline$|\overline{270}|$ & $\begin{array}{l}\text { Covid-19 badly impacts the economic world, busi- } \\
\text { nesses, and production houses. However, due to } \\
\text { less physical interaction during the pandemic, online } \\
\text { shopping trends observe a steep rise. }\end{array}$ & $\begin{array}{l}\text { A path to a trustworthy digital design and deployment to view current control } \\
\text { regulations and planned technological upgrades make business operationally } \\
\text { competent, robust, and competitive. }\end{array}$ \\
\hline $\mid 271$ & $\begin{array}{l}\text { Initial setup costs and recurring operational costs are } \\
\text { a big hurdle in small organizations starting an online } \\
\text { e-business. This study reviews these challenges. }\end{array}$ & $\begin{array}{l}\text { The article adopts the multi-case study model while adopting the qualitative } \\
\text { approach. The authors suggest that providing government-supported / funded } \\
\text { services to the small business organization regarding e-business hosting and } \\
\text { setup may better adopt e-business strategies by small organizations. }\end{array}$ \\
\hline $\mid 272$ & $\begin{array}{l}\text { The speed and abruption in processes of the smart } \\
\text { city require the associated systems to work in real- } \\
\text { time. The financial back-end is the backbone of any } \\
\text { city, and smart cities are not an exception. }\end{array}$ & $\begin{array}{l}\text { The paper comprehensively covers the various aspect of fintech work areas } \\
\text { and the methods and technologies for the inclusion of technologies in work } \\
\text { processes. The focus is the inclusion of AI to better fintech companies of the } \\
\text { future smart cities in audit, control, and operations to deliver intelligence-driven } \\
\text { services to the economy. }\end{array}$ \\
\hline $\mid 273$ & $\begin{array}{l}\text { Contracts are an important part of the economy, and } \\
\text { the smart economy is now widely using technologies } \\
\text { for record management and contractual operations. } \\
\text { The adoption of smart contact by fintech companies } \\
\text { results in faster and efficient contract-related opera- } \\
\text { tions. }\end{array}$ & $\begin{array}{l}\text { The authors use the two-stage game-theoretic model to test supply chain finance } \\
\text { theory for various fintech applications to gauge the performance and operational } \\
\text { strategies for both pre-shipment and post-shipment scenarios as per the smart } \\
\text { contract liabilities. The outcome is the guidelines for when to adopt smart } \\
\text { contracts and smart invoices and how they can be beneficial in a true sense to } \\
\text { the business. }\end{array}$ \\
\hline$|\overline{274}|$ & $\begin{array}{l}\text { The classical factories are now transforming to smart } \\
\text { factories to reap the benefit associated with smart } \\
\text { technologies in the work processes and facing chal- } \\
\text { lenges during adoption. }\end{array}$ & $\begin{array}{l}\text { The study outcomes are in three significant areas. At first, it proposes a } \\
\text { novel and cost-effective approach for product tracking. It then discusses data } \\
\text { acquisition systems for processes and discusses a fault detection system that } \\
\text { uses an artificial neural network. }\end{array}$ \\
\hline $\mid 275$ & $\begin{array}{l}\text { Covid-19, like a pandemic, can cause a major loss to } \\
\text { the production facilities due to lockdowns, etc. This } \\
\text { may result in a scarcity of important items. }\end{array}$ & $\begin{array}{l}\text { The research proposes the use of 3-D printing technologies to fight the shortage } \\
\text { of essential medical supplies. The study reviewed the production of biomaterials } \\
\text { and bioproducts using 3D printing, which will overcome the challenge of } \\
\text { producing medical items during lockdowns for covid control. }\end{array}$ \\
\hline
\end{tabular}

quality control, process, product defect detection, root cause analysis of issues, and other allied statistics gathered by using ML approaches [235]. Factories, traditional or smart, are usually divided into two major categories, i.e., Large scale manufacturing and small and medium-scale factories [278]. Figure 24 discusses prospective benefits and Edge gained by smart factories over the traditional ones in the case of both large-scale manufacturers and small-scale factories. [279] discusses the methodology for planning a smart factory from scratch. Large-scale and smart factories have enormous benefits in shifting their manufacturing plants to be based on smart technologies. This is the era of 3D Printing and additive manufacturing (AM) [280]. During the Covid-19 pandemic era, it was observed that the shortage of essential medical supplies was fulfilled using $3 \mathrm{~d}$ printing technology [275]. This creates a new dimension for manufacturing at a small scale, diminishing special manufacturing units' need for developing the products. Further to this, on-demand manufacturing and personalized/customized manufacturing also results in freedom from managing inventories [281].

Critical Analysis: Due to advancement in technologies and their incorporation into every sector related to human life, ways of manufacturing/production also get influenced. With the introduction of Just In Time production, Zero inventories, robotics, automation of production plants, $\mathrm{AI}$ and $\mathrm{ML}$, and 3D printing, the manufacturing sector faced further new chal- lenges. Those units that are jumping into the manufacturing have the Edge over the older traditional manufacturers. They can use the LeapFrog Technique to incorporate the latest technologies right from their production/manufacturing. This will give them the Edge to rapidly capture more markets, more accessible technology updates, introduce new trends, future-proofing their investments, and ultimately begin their journey to become a global business leader. On the other hand, those who choose not to upgrade will eventually diminish out from the scene. They will have a limited customer base, expensive comparative product prices, higher costs for technology upgrades, and a lack of customer satisfaction due to batch manufacturing. Table XI presents the Smart Economic Literature section tabularly.

\section{J. Smart Governance}

Smart cities cannot become smart enough unless they have smartness in their governance structure. Becoming a smartly governed city not only requires the use of technology but the incorporation of technology in the governance processes [282]. The evolution of smart cities also highlights that cities that run on information must have such information generation more smartly. This can only be done by reinventing the governance structure and replacing the manual theme of work into the more automated, technology-backed, and 
digital [283]. This transformation needs to be done in two aspects. i.e., technology-enabled automation in the processes and technology-backed intelligence in the decision support and management [284]. Key challenges in these aspects are the best use of technology to generate critical data on the required time for processing, the provision of information to the other processes of the governance structure, and the best utilization of that data in decision-making processes. The success factor is incorporated into a future smart city's critical performance indicators that are reshaping the governance structure. As well as allied governance processes at the local government departments and agencies and other stakeholders directly or indirectly [285].

The concept of electronic Government is now widely implemented in major cities of developed/developing countries. Past research brings the efficiencies at large by identifying and removing the issue and challenges [286]. However, it mainly revolves around the digitization of the classic governance structure. Electronic governance, however, became the foundation for the idea of smart governance when a delay in processes was identified, which was previously hidden before digitization. A significant portion of the future smart city will be linked directly or indirectly with the city's smart governance.

It is perceived that to get the true benefits of technology, the past, present, and future of every citizen. It must be visible to accommodate his/her role in the overall governance structure, even if it is on the receiving side of the action. It will also help in generating future requirements in the infrastructure to fulfill the needs. Smart governance must process the past ten years' trends to analyze and anticipate the next ten years' requirements and directions. Near to accurate guess requires quality and quantity of data and trends. The true challenge in this regard is to gather the pre-smart era data in the required form while keeping the citizens' privacy intact. Technologybacked technology-backed and technology-based government infrastructure is evolving, and it is on its way to having a mature, smart governance structure. Big data analysis is the key part of the future smart governance initiative enabling the solution of day-to-day problems and smart decision and planning management [287]. Big data analytic is the integrated part of every smart city aspect. However, its inclusion is more significantly highlighted by playing a supporting role in highlevel current and future decision making. Figure 25 presents the different dimensions of smart governance.

1) Smart Revenue: Revenue generation is the prime source of income for not only businesses and enterprises but also for government infrastructure too. To cover their running expenses and fund the public sector development projects, increase the overall revenue to cover the expenditures and costs. In the modern era,e of intelligent tools, technologies, and algorithms is integrated into revenue generation and management [291]. A bottom-up approach is recommended to transform the overall governmental infrastructure's various revenue generation systems to a smart technological-based system. An efficient, based on occupancy model, parking slot to the vehicle with dynamic cost model was suggested by [288] using ML techniques for competitive parking cost calculation. The same model can also

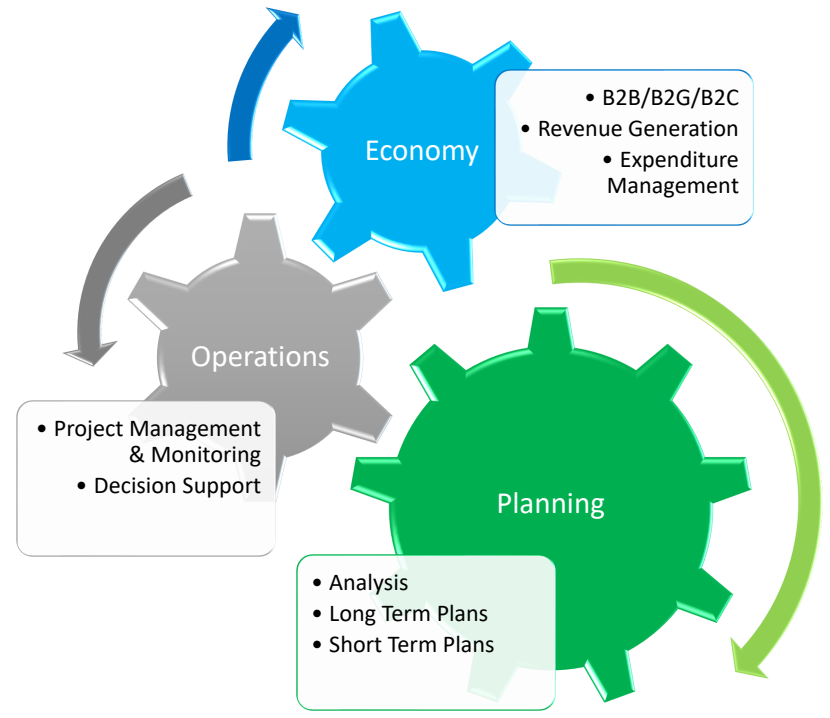

Fig. 25: Dimension of Smart Governance

be utilized with modifications to other scenarios of revenue collection.

Critical Analysis: Revenue generation is an essential aspect of city governance which has many facets in its implementation. The revenue may be generated from tax collections, utility usage payments, and fees for various facilities like parking, fines, and taxes collected from multiple revenue sources. Automation, the crucial factor for smart cities, helps smart cities ease management, application, collection, and auditing of revenue imposed, generated, collected, and spent. ML and AI can be utilized to the full extent to further dig deep into the different scenarios to find out the possible sources of revenue collection and the possible areas where the spent amount will yield the most beneficial outcomes for the city's betterment.

2) Safe City: In smart city governance, monitoring the city is a crucial task. An electronic, real-time, visual-based system is currently deployed in major cities worldwide, having central control rooms established to monitor the city's state. The defining of crucial points for the monitoring is the foundation for the safe city's whole architecture. This is usually done via predicting the risks and impacts study and adding entry/exit points and the invisible boundaries of the city area [289]. After finalizing monitoring points and technologies, the comprehensive, round-the-clock monitoring and analysis are executed for a proactive response. Automated and manual alert management is done upon identifying incident/crisis, which is then propagated to the appropriate stakeholders like health authorities, law management and enforcement authorities, and local Government for appropriate actions and containment of the situation. Figure 26 presents the safe city process flow.

Safe city design is the most crucial step in the overall lifecycle of future smart city safety. Safety must be incorporated into the design of smart city processes. The anticipation of 
TABLE XII: Summary of Smart Governance Literature

\begin{tabular}{|c|c|c|}
\hline Ref. & Proposed Work & Outcome \\
\hline $\mid 282$ & $\begin{array}{l}\text { In this paper, the authors addressed using smart urban } \\
\text { governance how to govern the urban challenges. }\end{array}$ & $\begin{array}{l}\text { Based on different available arguments, practitioners and researchers use smart } \\
\text { governance to address urban challenges and issues by starting from urban } \\
\text { issues, stimulating demand-driven smart governance modes, and determining } \\
\text { more technological intelligence and informally. }\end{array}$ \\
\hline 283 & $\begin{array}{l}\text { In this research paper, the authors address the role } \\
\text { of urban data boards in supporting city governments' } \\
\text { transport of smart city creativities. } \\
\text { The paper discussed the explosion of urban data } \\
\text { platforms }\end{array}$ & $\begin{array}{l}\text { Based on the set of different typologies and functions. The aim to set urban } \\
\text { platforms as a key site for developing some new models for smart city } \\
\text { governance. }\end{array}$ \\
\hline$|284|$ & $\begin{array}{l}\text { The paper aims to provide relationships and defini- } \\
\text { tions between concepts and smart governance like } \\
\text { electronic and smart Government in smart cities. }\end{array}$ & $\begin{array}{l}\text { Based on the literature review, the authors described the contribution to } \\
\text { developing a framework and defining smart city governance for building new } \\
\text { models that address different challenges of smart society information sharing, } \\
\text { collaborative governance, transparency, and citizen engagement. }\end{array}$ \\
\hline$[286$ & $\begin{array}{l}\text { The authors proposed to analyze the value of tra- } \\
\text { ditions in administration literature, the adaption of } \\
\text { traditions value for E-Government, and synthesize } \\
\text { three different prominent values. }\end{array}$ & $\begin{array}{l}\text { The authors conducted a qualitative survey that shows administrative efficiency } \\
\text { and concern for citizen engagement through a manager's value position. The } \\
\text { authors also discussed the imperative efficiency implications. }\end{array}$ \\
\hline 287 & $\begin{array}{l}\text { The authors proposed different dimensions which } \\
\text { help in assessing smart city performance. }\end{array}$ & $\begin{array}{l}\text { authors identified several well-accepted models for assessing the smart city } \\
\text { performance in the paper. Smart governance is one of the most important } \\
\text { dimensions from the identified dimensions. }\end{array}$ \\
\hline$|288|$ & $\begin{array}{l}\text { The authors proposed a novel mechanism for the } \\
\text { interested vehicle owners in finding the unoccupied } \\
\text { parking slot with the least overhead. }\end{array}$ & $\begin{array}{l}\text { The authors proposed an ML-based novel approached to predict unoccupied } \\
\text { parking slots used to assume possession for arriving vehicles. For training, } \\
\text { testing, and comparing available ML algorithms, a data set of on-street Seattle } \\
\text { city is used. }\end{array}$ \\
\hline$|289|$ & $\begin{array}{l}\text { The authors proposed a framework for the inter- } \\
\text { connected safe city and smart city concepts. The } \\
\text { proposed framework is similar concepts and common } \\
\text { to both. }\end{array}$ & $\begin{array}{l}\text { Well defined systems are always divided into features and layers. From various } \\
\text { points of view, compared works and number of studies, communication, and } \\
\text { experiences with executives responsible for and managing the smart city } \\
\text { development (SCD), describing system layers and concepts are presented for } \\
\text { the proposed framework. }\end{array}$ \\
\hline$|290|$ & $\begin{array}{l}\text { Review the importance of urban safety management } \\
\text { applications. }\end{array}$ & $\begin{array}{l}\text { The authors review academic literature related to safety and smart city in } \\
\text { general, analyzing the policy discoursed and commercial Smart City creativities, } \\
\text { around Rotterdam city. The authors also look at some 'Smart Urban Safety' } \\
\text { observes in the city, which is not publicly capable. }\end{array}$ \\
\hline
\end{tabular}

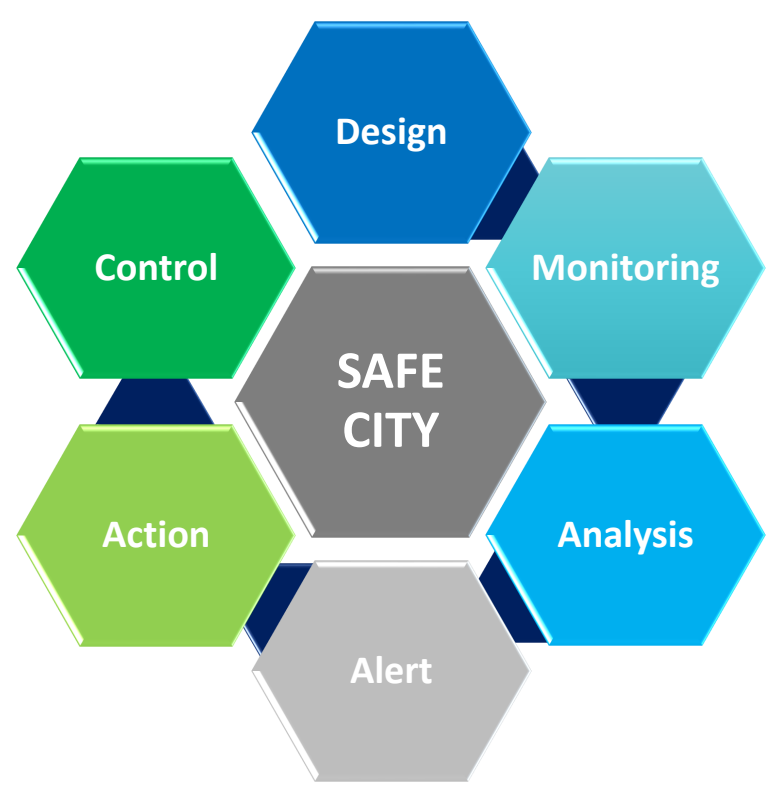

Fig. 26: Safe City Process Flow

threats and how to tackle them is the central question at this stage. More effectively and deeply, the thought process at this stage will result in security and safety at later stages. After the design, the monitoring process takes place to monitor the city processes $24 / 7$. The analysis was performed in near real-time using advanced AI techniques for possible pattern matching with the issue. After the match's occurrence, the alert is generated per the pattern's severity, and necessary actions were performed to neutralize the situation. The whole process is managed and controlled centrally.

Critical Analysis: Controlling crime is the primary function of any city, and smart cities are no exception. Maintaining a law and order situation is crucial for maintaining the quality of life in smart cities. Future smart cities will use advanced technologies to monitor the city situation to prevent any unpleasant situation before happening. It involves strict monitoring using sensors and cameras placed at appropriate locations, AI, and ML techniques to predict and act according to the model's outcome. Further, law enforcement officials use the latest technologies like drones, forensics tools, and big data analytics, resulting in faster analysis and investigations and often come up with more accurate investigations. More reliance and integration of technology in future smart cities will further ease the LEAs to make those cities SAFER.

3) Smart Law Enforcement: In the modern world, the inclusion of technology in law enforcement agencies' working increases many folds. Drone technologies, real-time monitoring, spying and bugging, crime investigation, and criminal tracking are now done more effectively and efficiently using the latest developments in information technology and digital forensics. $\mathrm{AI}$ is also used for crime suspect analysis and detection, and in a specific condition, the technology-based solution performs better than its counterpart human police officers [290]. The 
same technologies can also be used for safety and crime prevention by monitoring and analyzing the situation at the target place. It applies ML algorithms to identify possible crime-related activity. Using AI can update the nearby law enforcement personal or public as per the situation about the possible happening of the unwanted situation [292], [293].

The four main pillars for smart law enforcement operations are Prepare, Prevent, Protect and Pursue. To effectively use smart law enforcement's technological base, the operation and a human resource should be trained enough to incorporate the technological advancement into their day-to-day operations to improve accuracy and productivity. After the execution of this step, the next challenge is to prevent the crime before happening. ML, DL, and AI techniques can be used for context-aware situation monitoring and crime anticipating. In case of a crime, then law enforcement personal must be trained to protect human lives at any cost. The remaining importance goes to the property and governance. If the criminal successfully executes the crime, the law enforcement then uses safe city infrastructure to identify, detect and pursue the criminal to deliver justice to the victims. Figure 27 presents the four main pillars of smart law enforcement in a smart city.

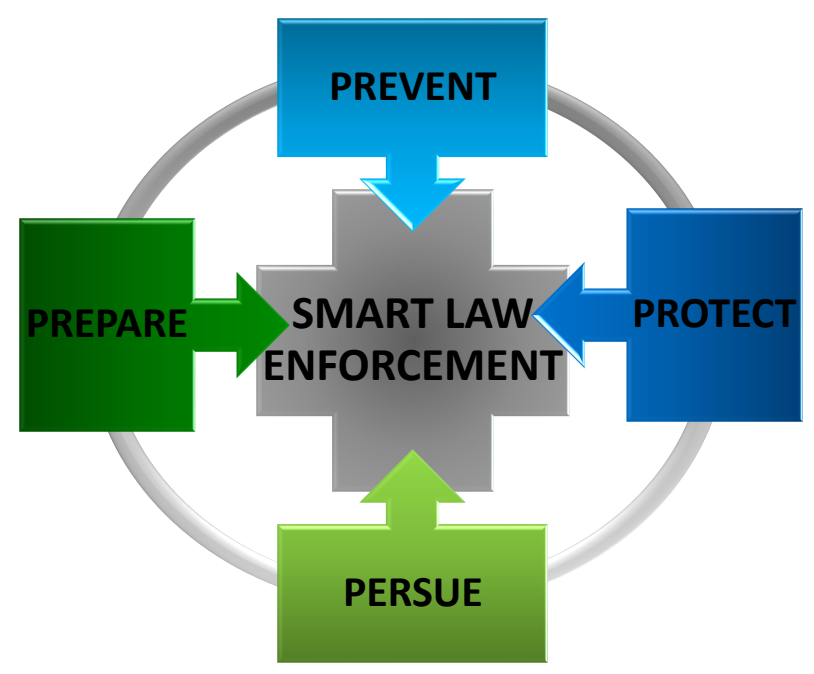

Fig. 27: Four Pillars Of Smart Law Enforcement

These pillars overlap and connect, creating a solid mesh bond to support and strengthen each other.

While creating bonds, contracts, and other agreements, the use of blockchain can result in verifiable and denial/tamper resistance built into it. This will results in less load on LEA's due to cases filed for financial and business frauds. UAE is using an utterly technology-based e-Trial within the judicial system. The role of UAV is also increasing in LEA operations and is widely used in operations by the LEA personnel in developed countries [247].

A video monitor is a critical part of the overall law enforcement activities. It requires monitoring (manually or intelligently) to deploy and manage optical surveillance equipment mainly based on fiber-optic communication. The work of [294] sheds light on recent trends and technologies in optical performance modeling using ML. The authors focus specifically on using ML-based optical performance modeling (OPM) and modulation format identification (FDI) by discussing key advantages gained after deploying optical networks in different scenarios.

Critical Analysis: Future cities will eventually see a decrease in crime rate due to increasing technological advancement, and it will become difficult to execute a crime successfully. The empowerment gained by the LEA will result in more focus on the prevention of crime and making it difficult for malicious persons to execute a crime. Monitoring and analysis solutions based on DL techniques and pattern recognition and facial recognition will update the local law enforcement and emergency service personals about the possible areas their services are required. Root cause analysis to prevent crimes can also be done using the data collected, stored, and analyzed during the operations. However, there is a cost to pay for such implementation, which compromises the persons' privacy. Privacy concerns are already raised on such monitoring activities and are increasing day by day. The major challenge in this regard is privacy-aware monitoring, analysis, and ML. Table $\mathrm{XII}$ presents the Smart Governance Literature section tabularly.

\section{K. Smart Environment}

Another primary target for a smart city is transformation by effective and efficient utilization of renewable energy sources, reduced waste production, and cycling and re-usage to reduce the environment's impact. This is one of the crucial pillars of a smart city and requires smart thinking while planning and designing any future smart city focusing on impacts and outcomes on the People, Places and Planet [295].

The smart environment is the broader term that covers water and air quality, pollution, weather, radiation, waste, health, and natural disasters. Under this scope, a grid of sensors, monitors, analyzers, and actuators worked together to achieve a specific sub-task. These sub-tasks are categorically divided into two domains, i.e., environmental resource management and environment quality and protection management.

1) Green Smart Cities IoT: It is essential to take the necessary steps to promote energy efficiency and prevent energy depletion from accomplishing green IoT implementation. Using clustering, we can extend the lifetime and efficiency of networks like IoT, depending on the quality of clustering schemes selection [296]. IoT is an eco network, not only used for transferring data into and between different networks; it is connected with Cloud Computing and Big Data to provide intelligence and recognize connected devices' behaviors. [303] Presents a comprehensive overview of the green IoT included its applications, challenges, concepts, and technologies. After increasing the popularity of IoT, IoT-based applications and devices like smart city applications, low power, and long-range wireless-based connectivity solutions are increased day by day [297]. To improve the sensor-based communication in SMART cities [296] presented an IoT method for constructing a green wireless sensor network (GWSN) with minimal power usage, minimum radio frequency impacts, and better performance. [298] applied a convolutional neural to of a green IoT network for monitoring. Figure 28 presents the lifecycle of green IoT in smart cities. 
TABLE XIII: Summary of Smart Environment Literature

\begin{tabular}{|c|c|c|}
\hline Ref. & Proposed Work & Outcome \\
\hline 295 & $\begin{array}{l}\text { How to achieve Sustainable and Smart Environments } \\
\text { using "Smart Thinking" in city development using } \\
\text { case study principles of Hong Kong. }\end{array}$ & $\begin{array}{l}\text { By reviewing and assessing the effort in making a sustainable and smart } \\
\text { city by Hong Kong, the research paper enables and aware other researchers } \\
\text { regarding the overall future positioning and quality of smart cities in achieving } \\
\text { a sustainable and smart Hong Kong. }\end{array}$ \\
\hline $\mid 296$ & $\begin{array}{l}\text { Development of Green Wireless Sensor Network } \\
\text { with the help of IoT Network to improve sensor- } \\
\text { based communication quality in smart cities. }\end{array}$ & $\begin{array}{l}\text { The research paper proposed a new algorithm called Improved Adaptive } \\
\text { Ranking Based Energy-Efficient Opportunistic Routing Protocol. The protocol } \\
\text { is based on relative distance, regional density, and residual energy. In improving } \\
\text { energy efficiency, the last node, half node, and first node death are the main } \\
\text { challenges. }\end{array}$ \\
\hline [297] & $\begin{array}{l}\text { For energy-efficient connectivity, the authors pro- } \\
\text { posed using a multi-hop LoRa network in a smart } \\
\text { city environment. }\end{array}$ & $\begin{array}{l}\text { Using a cased study, the authors compare and evaluate the single and multi- } \\
\text { hop LoRa network in terms of energy efficiency and range extension using } \\
\text { the evaluation packet reception ration method for different spreading factors, } \\
\text { transmission powers, and distances. Results of the experiments show that the } \\
\text { multi-hop LoRa network enhances coverage and saved significant energy. }\end{array}$ \\
\hline$|298|$ & $\begin{array}{l}\text { Using a convolutional neural network (CNN), the } \\
\text { authors proposed the intelligent safety surveillance } \\
\text { (ISS) method. For detecting workers' helmets, CNN } \\
\text { is a supervised auto technique. }\end{array}$ & $\begin{array}{l}\text { The authors trained the ISS model using the CNN technique and labeled the } \\
\text { datasets. The authors redesign the CNN framework and loss functions which } \\
\text { are based on YOLOv3. Based on the specific detection evolution metrics, the } \\
\text { proposed ISS method is tested. The experiment results show that the proposed } \\
\text { ISS technique enables the different models to learn labeled information from } \\
\text { datasets. }\end{array}$ \\
\hline$|\overline{299}|$ & $\begin{array}{l}\text { The authors proposed and developed a green acces- } \\
\text { sibility index method to represent the efficiency of } \\
\text { assessing public green spaces. }\end{array}$ & $\begin{array}{l}\text { To improve trip time and optimize path choice time estimation, a map service } \\
\text { is adopted. To estimate green space accessibility, an improved method is raised } \\
\text { at a fine scale. The technique improved the capacity of public urban green } \\
\text { spaces to inform planning. }\end{array}$ \\
\hline$|\overline{300}|$ & $\begin{array}{l}\text { The authors proposed green energy to give IoT } \\
\text { devices power and revolutionarily wireless charging. }\end{array}$ & $\begin{array}{l}\text { The authors proposed green IoT in three steps: ambient energy harvesting, } \\
\text { Energy wireless charging, and Energy balancing. }\end{array}$ \\
\hline $\mid 301$ & $\begin{array}{l}\text { The research paper proposed privacy and security } \\
\text { issues challenges in IoT-based agriculture. }\end{array}$ & $\begin{array}{l}\text { The authors summarized existing surveys on smart agriculture and described a } \\
\text { four-tier green IoT framework for agriculture. The authors categorize included } \\
\text { integrity, authentication, privacy, availability, and confidentiality in the classi- } \\
\text { fication of threat models used in green IoT. }\end{array}$ \\
\hline $\mid 302$ & $\begin{array}{l}\text { For effective waste disposal and recycling using a } \\
\text { DL strategy, a novel technique is proposed for waste } \\
\text { management. }\end{array}$ & $\begin{array}{l}\text { To train a dataset YoLOv3 algorithm is utilized in the Darknet network. Six } \\
\text { objects (organic waste, plastic, cardboard, paper, glass, and metal) have been } \\
\text { used for a trained dataset. Using YoLov3-tiny detection task and comparative } \\
\text { assessment is performed. The results show that the YOLOv3 framework yields } \\
\text { acceptable simplification capability for all classes with the variability of waste } \\
\text { material. }\end{array}$ \\
\hline
\end{tabular}

\section{GREEN IOT DESIGN}

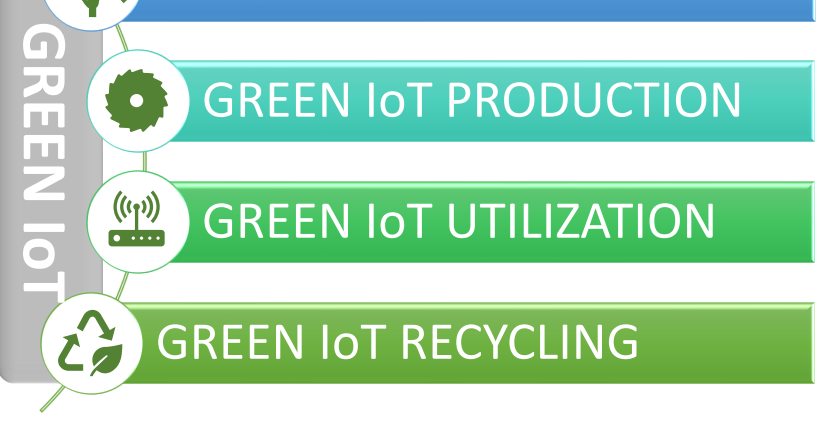

Fig. 28: Green IoT Lifecycle

As urbanization results in a reduction of ecological land and an increase in climate disturbance, keeping in view the danger to nature, the metropolitan-level green urban spaces are promoted in developed countries [299]. The idea and empowerment behind green's growth are to make the climate impact as little as possible on implementing technology to get the most benefits out of it. The most devastating one is brown energy for powering up / charging these devices, which cost- wise damage is most prominent among all [300]. In the field of smart agriculture, green IoT-based agriculture is proposed through the implementation of four layers at the agriculture sensor level, fog computing level, the core layer, and a cloud layer in [301]. The overall green IoT life cycle is based on the green by design, green during production, green in utilization, and green while recycling, i.e., having minimum to no impact on the environment during all these phases [303].

The new enhancement in IoT felt after the continual rise in hacking incidents involving IoT devices realizes the need to develop secure IoT (SIoT). Security by design is the longterm solution for battling the security risks aligned with IoT devices in smart factories, smart homes, smart transportation, smart fintech, and smart healthcare, to name a few. This is the central concept behind [304] research motives.

IoT is composed of embedded systems with communication to the internet, making the true green IoT achievable. The communication part must be given importance for efficiency and lesser resource hungry. [305] provides a comprehensive overview of the latest research in the physical and crosslayer integrated systems. The physical layer section discusses coded modulation schemes based on P-LDPC. The cross-layer section discusses the JSCC, JCPNC, and JCC-DCSK to make communication efficient and green.

Critical Analysis: Excessive use of technology results in extra resource usage, which ultimately results in many impacts 
on climate. As most solution implemented in the smart city is heavily based on IoT, optimization efforts are made to minimize the adverse effects of such implementations on the environment. These impacts may be direct or indirect, and both are hazardous in the long run. To control the negative effects of technologies, the challenge was accepted to reduce the negative impact using various corrective and preventive measures to make the technologies green enough to show the difference in past and present. The major work was done in the field of power consumption of IoT devices and the network area. Devices, protocols, and algorithms are devised to view the challenge of minimizing power consumption to increase the product work-life and keep in view the harsh environment devices may have to face after deployment and the reduction in impact on climate minimizing electromagnetic emissions.

2) Smart Waste Management: Once considered a responsibility to fulfill clean environment requirements, Waste management is evolving as a new opportunities highway. The improvement in the quality of life and advancement in technology often results in a degradation in the climate [302]. In the city, innovative or conventional, it was considered a challenge to manage the life cycle of waste, i.e., waste generation, collection, transportation, separation, and treatment. Treatment may include recycling, incineration, and land-filling [306]. The initial implementation of smart waste management revolves around the inclusion of technology in the whole process. Researches in different areas now make it possible to generate products from waste in different ways. Wastewater is now treated and provided for irrigation purposes. In some areas, electricity is produced while incinerating the waste at the city level. Plastic from the waste is not molded and reformed for its later utilization in various ways. A relatively more minor portion of waste that is not utilizable is now left at the end of the cycle for disposal. Figure 29 presents the waste management system in a smart city.

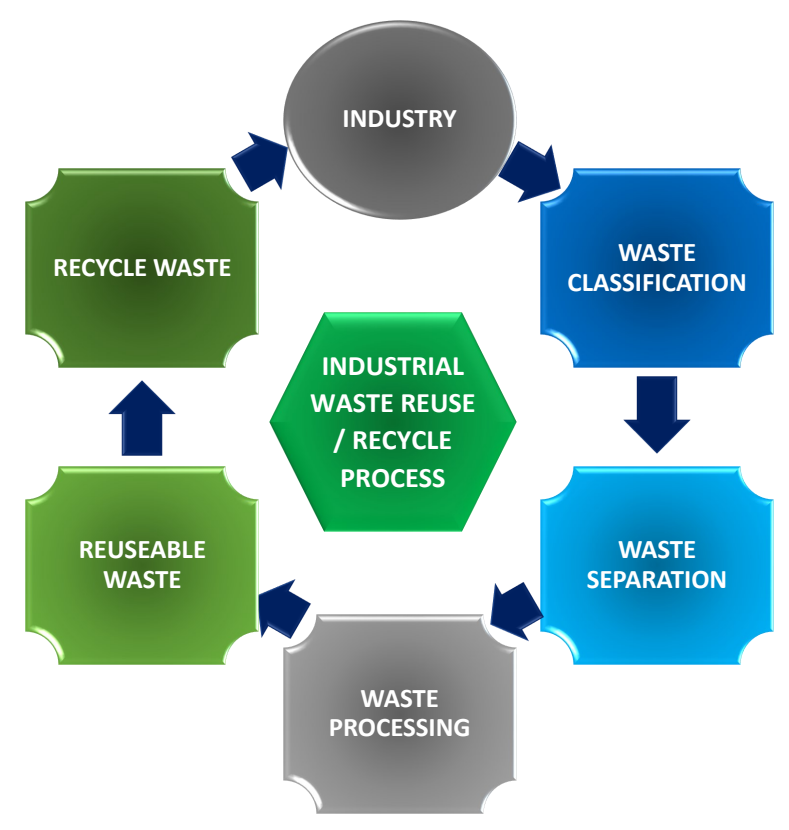

Fig. 29: Waste Management in Smart City
Automation in waste management for future smart cities requires a technology-based automated and coordinated system to analyze, manage and reprocess/recycle the waste. Sensorbased IoT devices are in use for the same in today's industries [307.

Critical Analysis: The smart city's waste management will be an iterative process where the waste will be processed and reused for other activities. The approach in the future smart city regarding waste would be REUse, REDuce and RECycle. Initially, waste management was considered overhead, but now it transforms entirely differently. Waste management now becomes a revenue generation stream where industrial waste is recycled and reused in several industries and saves crucial foreign reserves. Another advantage is the lesser harm to the climate due to lesser production of waste after reuse / recycle. Waste processing is now turning towards an industry that generates wealth out of the waste. Advanced technologies will eventually transform this industry into providers of processed raw material to other industries and reduce the waste output of the smart cities.

3) Smart Power: Electricity is the commodity of life, which becomes an integral part of human life, like air, food, or water. Not only do the essential gadgets and communication tools need power, but the recent shift on green transportation creates the need for electric power to run electric vehicles. Hence, the city, without power, cannot manage its affairs and operations. The technology dependence of smart cities requires the supply of enough and uninterrupted energy as per requirements. One of the best solutions to produce green energy with minimal impact on the environment is solar energy, which provides a sustainable and continuous (daytime) energy source at small and large scale and supports modular development/implementation approach [295]. Fulfilling the future smart city's energy needs will be the big challenge to solve as lack of adequate power, even for a short time, will result in significant disruption [308]. As a smart city's main requirement is to have a reliable, energy-efficient, and continuous supply of power, the complexity of such uninterrupted power supply increases by many fold [309]. [310] suggests using a clustering technique to resolve complexity in the scalability of the power grids and other CPSs for future smart cities. [311] emphasize the requirements of an electrical energy system that is prone to unwanted issues and incidents like cyber attacks and physical risks for future smart cities.

There is a need to use advanced AI and ML approaches in every aspect of power generation, from requirement analysis to end-users utilization. This will optimize the utilization of existing generation approaches and prevent energy starvation [312]. Further to this, power generation and distribution companies must also enhance their technical competence to remain competitive in the relevant market. Gaining operational efficiencies can be one of the crucial aspects in the power sector of future smart cities. It can be attained using hybrid tactics based on management, data analytics, and trend analysis.

Critical Analysis: Power acts as the blood in smart cities. Due to the complete technology base back-end in every aspect of the smart cities, the power outage can standstill the city's running life. On the other hand, the power requirement is 
an ever-increasing phenomenon, and with the advancement in technologies, more power requirements in anticipated in the coming years. The growth in power generation is not at that pace and also possesses cost overheads. The futureproof approach generates most of the required energy using renewable technologies from natural sources such as wind, water, and the sun. Further inclusion of AI and ML techniques to monitor the demand and supply can help achieve optimum operational efficiencies. Security and threat management of the power infrastructure is also crucial as power outages may negatively impact the smart city. Table XIII presents the literature on the smart environment.

\section{Open Research Issues and Future Directions}

System of Systems is the phenomenon of collecting complex systems that coordinate with each other and work collectively and cumulatively to appear as a single system to the enduser. The internal systems pool their resources and capabilities to offer more services as a joint single entity/system. Smart cities are one such example of a system of systems. With the wide range of potential research and applications in smart cities, several open issues and challenges need to be considered. Researchers are working on the architectures and models of smart cities to deploy, integrate and build smart city applications. Examples of these broad range issues included inter-connectivity, communication and security, and smart cities' seamless integration. Several smart city approaches and policies are also under investigation. Governing bodies of smart cities are already working on the operation guidelines, regulations, and policies. These efforts will guide and help access more smart cities' applicable technologies, improve operability, smooth development efforts, services, simplify seamless integration with other types of systems, and finally fully integrate different applications in smart cities.

\section{A. Technical Risks and Challenges}

Technology advancement is an opportunity as well as an issue in the paradigm of smart cities. The increasing pace in the invention of new technology lacks standardization. It is possible only when one has a stable technology to benchmark and design or define minimal to maintain. Further, the latest technologies may have issues in their integration with the existing technologies. They may require changes within the established systems that will again need to assess the system's stability and functionality before incorporating.

In communication, smart cities' fundamental components and computing, storage, and reporting have been faced and dealt with different challenges. A careful analysis is required in terms of node communication component cost, network cost, battery survival, data throughput, real-time communication requirement, coverage area, range of communication, mobility requirement of nodes, deployment model, and latency management [313].

The birth of IoNT is a significant breakthrough in the recent past which adds miniaturization to the size of IoT devices and makes their wide-scale implementation further easy. Multiple sensors getting a more precise status of the aspect without bringing significant addition to the size and power consumption requirements [231]. However, adding more nodes in the system means more data to handle in the compute and network layers.

1) Resources: Resources required to convert a city's infrastructure into a smart one require resources and investments in technological, financial, and human sectors.

2) Implementation: Generally, one of the two approaches is followed for smart city projects (i.e., top-down approach and bottom-up approach). The top-down approach is used when converting an established city from tradition to smart. The top tiers of the systems are converted to smart, and then based on their data requirements, the systems beneath them are identified and converted gradually, resulting in the whole transformation taking years. A bottom-up approach is used in designing a new smart city project. The system's components are designed by incorporating the smartness in them, and then their outputs are integrated and utilized at the higher level for further processing and utilization in decision support. The selection of the best-suited methodology is essential for a practical smart city project.

3) Technology Reliance: The smart city concept is nothing but the inclusion of technology in a smarter way to join different technology-based solutions within the various aspects of city life to combine the effort in a coordinated manner to achieve a better standard of living in that city. These included technology has some hazards and threats too, which, after their utilization into smart city functions, added those threats to the horizon of smart city infrastructure.

\section{B. Open Issues based on the Literature Review}

With the wide range of potential research and applications in smart cities, we highlight several open issues, challenges, and practical future directions based on the literature review.

1) $6 G$ Networks: The need for a faster network connection is the result of $5 \mathrm{G}$. However, the recent development of technology is rigorously progressing in terms of processing. It requires an even faster network connection to keep the synchronizations of the system, and this requirement gives rise to $6 \mathrm{G}$ networks. The supreme connectivity abilities of 6G inefficiency and faster bandwidth will experience virtual and augmented reality (VR/AR). The aspiration to impart at ever higher information rates will not ever stop. To achieve at terabytes each subsequent information rate, it is inescapable to work at ever more elevated recurrence groups. Enormous scope receiving antenna exhibits is expected to beat the expanded way misfortune and other spread marvels need the help of different equipment parts, including signal blenders, ADCs/DACs, and power amplifiers. The significant expense and force utilization of these segments at the mmWave and $\mathrm{THz}$ groups make it hard to receive traditional handset structures, thus influencing the design of signal processing algorithms. To adequately create such complex frameworks, cooperation among the equipment and algorithms domains will be required; that is, the equipment calculation co-plan should be supported. 
2) Big Data 2.0: The potential for data will probably continue growing. The prime grounds behind this are the extreme increment in handheld apparatus and internet-connected apparatus predicted to rise within an empirical purchase. SQL will continue to be the norm for information investigation, and Spark will emerge, which could emerge as the numerical instrument for information investigation. Programs such as investigation minus the current clear presence of an analyst have been put to shoot more than together with Micro Soft and sales-force both not too long ago declaring attributes permitting non-coders to generate programs for seeing data. According to IDC, all company analytics applications include intelligence at which is needed from 2020. To put it differently, it might be explained that prescriptive analytics will soon likely probably be constructed into the business program. Apps such as Kafka and Spark will let end customers create conclusions inside real life. ML will possess a much more significant part in data prep and predictive investigation from businesses from the forthcoming times. Security and privacy challenges linked to huge statistics will rise; also, from 2018, $50 \%$ of organization integrity violations will undoubtedly soon probably be associated with info. Chief info officer will probably be quite a frequent sight in most organizations in the long run though it is thought it will not continue long term. Autonomous robots and agents, autonomous cars, digital private assistants, and also intelligent apparatus are going to undoubtedly probably likely soon become a vast tendency. Substantial data ability dip will certainly reduce from the forthcoming times since can be observed those times. The International Institute for Analytics forecasts that employers will probably work with internal and recruiting teaching for budding statistics boffins to receive their issues carried out. Organizations will shortly have the ability to get calculations instead of system them and insert their data. Existing products and providers such as Algorithmia, DataXu, and also Kaggle will rise to an enormous scale; this will be, algorithm economies will probably arise. More organizations will take to draw their earnings out of their data. The difference between comprehension and activity from big numbers can probably diminish, and much more energy will be awarded to gaining execution and insights instead of collecting big data. Speedy and technical data will probably replace colossal information. Organizations are to request the most relevant issues and make much better usage of this information, and their vast numbers are fresh nowadays.

3) WiFi 7: 802.11ax, the usage of high modulation orders, proactively encouraging Aid of $320 \mathrm{MHz}$ broadcasts, that is twice the $160 \mathrm{MHz}$ of all 4096-QAM- upward from 1024QAM from 802.11ax- and also the feasibility of numerous when in comparison to Wi-Fi 6, Wi-Fi 7 (or 802.11 Function as) may additionally utilize for the Wi-Fi standard. However, lead improvements on Wi-Fi to include multi-band/multichannel aggregation and performance and deliver increased range and electrical strength efficacy, far superior disturbance mitigations, greater power density, and greater cost-efficiency. The Wi-Fi 7th generation can also be known as Wi-Fi's substantial throughput because of its estimated capacity to aid up to $30 \mathrm{Gbps}$ Throughput, about three times speedier than Wi-
Fi 6. Additionally, there is a lot of all proposed characteristics resource components, like collections of OFMDA tones.

4) Industry 5.0: Power for running a smart city is a fundamental necessity. However, there is no detailed research on the different power sources such as nuclear, wind, hydro, and thermal energies to light up smart cities. A detailed comparative analysis of power sources is proposed by the researchers in the coming future work. Smart cities are ways considered to be revolutionizing in technical ways, but the trends in smart cities govern some non-technical ways. The objects' art and design are some of the most highly neglected topics from being reviewed by the researchers when doing literature work on smart cities. Art and design significantly impact smart cities' visualization regarding the buildings, architecture, and interior. The progress of smart cities is highly dependent on satellites for IoT and inter-connectivity around the world. However, the operational medium of the satellites is also in space outside the planet Earth. Thus, the researchers intend to conduct future research on the effects of astronomy on human-made things and how they can be more efficient for future smart cities. The need for Industry 5.0 arises when it is observed that efficiency in the workflow can be further extended to an ultimate optimum level when HCI is incorporated in industries. The robustness of smart systems in the cities also arouses threats to precious information. The research directions must be primarily sketched in the way of cyber defense mechanisms, i.e., intrusion detection systems and intrusion prevention systems.

5) Advanced Robotic Systems: Next-generation software in robotics and mechatronics will demand essential, adaptive, streamlined, and significantly more economical technology. Technologies can be accessible due to the digital removal of small-level opinions controller, sensors, cabling computers. Conventional electrical motors, for example, terminal motors and drive-train, are not very appropriate for Boolean technology, even although, due to the fact they get intricate, cumbersome in addition to high priced. These kinds of automation were not recognized and therefore have been struck with chief topics of usability. So in the upcoming creation, the most current fad that's the Web of stuff will undoubtedly be utilized with those technologies and devices that we utilize inside our daily lives to do our job better with hardly any relief. Two-way communication might be said as full-duplex communication, exactly wherever the system interacts with still yet another apparatus of its work and own so. It continues to be referred to since the Web of objects and will certainly be known as an interconnection of devices or items.

6) Advanced Cyber-Security: Security is the fundamental requirement of every aspect of life. Thus, to enhance the security of the proposed literature, a suitable environment shall be developed to test out the loopholes in current state-of-the-art models. Furthermore, the work will be extended to assess the vulnerabilities of that critical system that are not reviewed so far. Also, it is noted that the side-channel attacks are highly neglected in the smart cities literature frameworks. For this deficiency, the researchers intend first to identify the exposed systems in public that can cause a data breach. Current stateof-the-art techniques and newly devised proposed approaches 
shall be tested on that cybersecurity threat to testify the strength of the approaches.

7) ECO-Friendly Technologies: Smart plantation and crossproduct vegetation are some of the commonly practiced techniques in farms for smart revenue. However, there is still a big room for research in making this vegetation nutritious for the residents of smart cities. Thus, the researchers also intend to conduct detailed research over the cross vegetation for envisioning better health of citizens of a future smart city. In medicine, the researchers intend to review the chemical compositions of medicines and study their side effects on the modern smart city inhabitants according to their activities. In order to make smart agriculture more robust and dynamic, use of nano technology specially IoNT devices can play a vital role in the context of smart cities. This area has huge study requirements to make an intelligent smart environmental protection and improvement system with the reliance on IoNT sensors data.

8) Artificial Intelligence: In the AI-based modernization of smart cities, we identify additional spaces where automation and sophistication can be done. For this purpose, thorough research shall be conducted to test out the conventional methods, current state-of-the-art optimized techniques, and the newly envisioned proposed approaches. Future is about resiliency in ML, context awareness in ML, federated learning, federated deep reinforcement learning, explainable AI, self-supervised learning, quantum computing, context-aware natural Language Processing, transfer learning, extended reality. In HCI, it is being observed that human brain-linked technology is being highly under trending research topics, but the connections of this technology are still missing in smart cities. Thus, human brain optimized and controlled automated technologies shall be reviewed in detail to make smart cities even more robust. To make work efficient, automated, and eco-friendly, electric motors and AI vast open room for research to make life smarter and healthier.

9) Healthcare and Lifestyle: The prime purpose of smart cities is to bring comfort for human beings and efficiency in current state-of-the-art techniques and technologies available for humankind to live a better quality of life. Smart cities are initially envisioned to upgrade daily life living standards in terms of ease, efficiency, and security. The design has also become a mode of human interaction with the information since it makes the information more engaging. The researchers intend to thoroughly review these fields to study the revolutionizing behavior of art, design, and the fashion industry. The world is facing calamities and pandemics since the beginning of life. Therefore, to make the smart cities more sustainable towards disastrous in-comings, an adequately devised backup mechanism should be envisioned to encounter uncertain circumstances. This highlights a great field of research when the recent pandemic, COVID-19, is regarded, and no such preventive mechanism had been found at the initial level. The inclusion of IoNT in H-IoT can results in increase in spread as well as depth of the state of the art technology based healthcare facilities and medical tasks that were previously considered impossible before the nano-tech age. Effective design, development and operations of such nano sized gadgets is a challenge that needs careful attention in order to make humans enjoying healthier life in the future smart cities.

10) Context and Situation Awareness: Within future smart city systems, situational awareness is the critical intelligence that can be beneficial for endless implementation. Situational awareness is most utilizable for law enforcement and crime control. It can also help in health care facility monitoring and may act as a positive add-on for smart nursing homes and smart childcare facilities. The activity tracking, identification, and context-aware monitoring will bring in-depth changes in how many systems in the smart city interact with the city environment. Architecture, analysis, and adoption are the three A's of situational awareness techniques, which will see comprehensive research and development shortly.

11) Concept Transportation: Transportation systems evolve in various perspectives, including eco-friendly characteristics, smart features, and multi-terrain maneuvering capabilities. Moreover, significant research is going on to enable road transportation systems to fly. In future transportation, it is intended to introduce EV systems in heavy vehicles along with robust catalytic converters to maintain the power while being eco-friendly. Moreover, a whole new routing system for flying vehicles must be devised to maintain a uniform flow.

\section{CONCLUSION}

The concept of future smart cities is vital to improving citizen's well-being and quality of life. A smart city is such a broad concept that doing it in steps is more fruitful for traditional cities. However, to design a new city, planning is based on technology to make it a smart city from scratch will be a better option to adopt. The world is scurrying towards implementing technologies to ease the governance, management, and control of the city and ease and quality of life of citizens. This paper discussed requirements for future smart cities, application frameworks, 360-degree coverage requirements, technology challenges, and implementation strategies are discussed briefly to make them a baseline standard for future smart cities. We reviewed the latest state-of-the-art studies and their crucial point of interest. This paper also combines the vital technologies/areas related to smart cities in a single paper to provide a way to look into the smart cities as a single system of systems instead of separate independent systems as current research focusing on a single aspect of a smart city. This 360-degree viewing/covering smart cities will eventually result in more research in the area while viewing the smart cities as a whole single system composed of different complex systems as mentioned in the taxonomy presented in this paper. Furthermore, we compared the proposed future smart city architecture with current state-of-the-art studies. We notice that state-of-the-art lacks in several areas of research which majorly include $6 \mathrm{G}$ networks, WiFi-7, industry 5.0, robotic systems, human well-being, HVAC, pantry backup, calamity backup that are inevitable for future smart cities. We also discussed the different technological challenges of future smart cities. 


\section{REFERENCES}

[1] C. Perera, Y. Qin, J. C. Estrella, S. Reiff-Marganiec, and A. V. Vasilakos, "Fog computing for sustainable smart cities: A survey," ACM Comput. Surv., vol. 50, no. 3, Jun. 2017. [Online]. Available: https://doi.org/10.1145/3057266

[2] Y. Liu, "The application of human-computer interaction in smart city planning and design," in International Conference of Design, User Experience, and Usability. Springer, 2018, pp. 101-111.

[3] B. Knowles, O. Bates, and M. Håkansson, "This changes sustainable hci," in Proceedings of the 2018 CHI Conference on Human Factors in Computing Systems, 2018, pp. 1-12.

[4] E. Okai, X. Feng, and P. Sant, "Smart cities survey," in 2018 IEEE 20th International Conference on High Performance Computing and Communications; IEEE 16th International Conference on Smart City; IEEE 4th International Conference on Data Science and Systems (HPCC/SmartCity/DSS), 2018, pp. 1726-1730.

[5] C. S. Lai, L. L. Lai, and Q. H. Lai, Smart City. Cham: Springer International Publishing, 2021, pp. 1-171. [Online]. Available: https://doi.org/10.1007/978-3-030-52155-4_1

[6] ISO/IEC. Iso/iec 30182:2017(en) smart city concept model guidance for establishing a model for data interoperability. [Online]. Available: https://www.iso.org/obp/ui\#tiso:std:iso-iec:30182:ed-1:v1:en

[7] F. Xhafa, B. Kilic, and P. Krause, "Evaluation of iot stream processing at edge computing layer for semantic data enrichment," Future Generation Computer Systems, vol. 105, pp. 730 - 736, 2020. [Online]. Available: http://www.sciencedirect.com/science/article/pii/ S0167739X19321296

[8] M. M. Alam, H. Malik, M. I. Khan, T. Pardy, A. Kuusik, and Y. Le Moullec, "A survey on the roles of communication technologies in iot-based personalized healthcare applications," IEEE Access, vol. 6, pp. 36611-36631, 2018.

[9] C. S. Lai, Y. Jia, Z. Dong, D. Wang, Y. Tao, Q. H. Lai, R. T. Wong, A. F. Zobaa, R. Wu, and L. L. Lai, "A review of technical standards for smart cities," Clean Technologies, vol. 2, no. 3, pp. 290-310, 2020.

[10] R. Zhao, T. Stincescu, E. E. Ballantyne, and D. A. Stone, "Sustainable city: Energy usage prediction method for electrified refuse collection vehicles," Smart Cities, vol. 3, no. 3, pp. 1100-1116, 2020.

[11] H. Baali, H. Djelouat, A. Amira, and F. Bensaali, "Empowering technology enabled care using iot and smart devices: A review," IEEE Sensors Journal, vol. 18, no. 5, pp. 1790-1809, 2017.

[12] Y.-S. Jeong and J. H. Park, "Security, privacy, and efficiency of sustainable computing for future smart cities," JIPS (J. Inf. Process. Syst.), vol. 16, pp. 1-5, 2020.

[13] A. C. Şerban and M. D. Lytras, "Artificial intelligence for smart renewable energy sector in europe-smart energy infrastructures for next generation smart cities," IEEE Access, vol. 8, pp. 77 364-77 377, 2020

[14] G. Javadzadeh and A. M. Rahmani, "Fog computing applications in smart cities: A systematic survey," Wireless Networks, vol. 26, no. 2 , pp. 1433-1457, 2020.

[15] L.-D. Radu, "Disruptive technologies in smart cities: A survey on current trends and challenges," Smart Cities, vol. 3, no. 3, pp. 1022 $1038,2020$.

[16] L. U. Khan, I. Yaqoob, N. H. Tran, S. A. Kazmi, T. N. Dang, and C. S. Hong, "Edge computing enabled smart cities: A comprehensive survey," IEEE Internet of Things Journal, 2020.

[17] A. N. Muhammad, A. M. Aseere, H. Chiroma, H. Shah, A. Y. Gital, and I. A. T. Hashem, "Deep learning application in smart cities: recent development, taxonomy, challenges and research prospects," Neural Computing and Applications, pp. 1-37, 2020.

[18] M. Bilal, R. S. A. Usmani, M. Tayyab, A. A. Mahmoud, R. M. Abdalla, M. Marjani, T. R. Pillai, and I. A. Targio Hashem, "Smart cities data: Framework, applications, and challenges," Handbook of Smart Cities, pp. 1-29, 2020.

[19] S. Bhattacharya, S. R. K. Somayaji, T. R. Gadekallu, M. Alazab, and P. K. R. Maddikunta, "A review on deep learning for future smart cities," Internet Technology Letters, p. e187, 2020.

[20] M. Songhorabadi, M. Rahimi, A. M. M. Farid, and M. H. Kashani, "Fog computing approaches in smart cities: A state-of-the-art review," arXiv preprint arXiv:2011.14732, 2020.

[21] Y. Zhou, F. R. Yu, J. Chen, and Y. Kuo, "Cyber-physical-social systems: A state-of-the-art survey, challenges and opportunities," IEEE Communications Surveys \& Tutorials, vol. 22, no. 1, pp. 389-425, 2019.
[22] H. Wang, H. Zhao, J. Zhang, D. Ma, J. Li, and J. Wei, "Survey on unmanned aerial vehicle networks: A cyber physical system perspective," IEEE Communications Surveys \& Tutorials, vol. 22, no. 2, pp. $1027-1070,2019$.

[23] Y. A. Qadri, A. Nauman, Y. B. Zikria, A. V. Vasilakos, and S. W. Kim, "The future of healthcare internet of things: A survey of emerging technologies," IEEE Communications Surveys \& Tutorials, vol. 22, no. 2, pp. 1121-1167, 2020

[24] A. Kirimtat, O. Krejcar, A. Kertesz, and M. F. Tasgetiren, "Future trends and current state of smart city concepts: A survey," IEEE Access, vol. 8, pp. 86448-86467, 2020 .

[25] P. Wegner, "Interoperability," ACM Computing Surveys (CSUR), vol. 28 , no. 1 , pp. $285-287,1996$.

[26] M. D. Hill, "What is scalability?" ACM SIGARCH Computer Architecture News, vol. 18, no. 4, pp. 18-21, 1990.

[27] A. B. Bondi, "Characteristics of scalability and their impact on performance," in Proceedings of the 2nd international workshop on Software and performance, 2000, pp. 195-203

[28] J.-C. Fernandez, L. Mounier, and C. Pachon, "A model-based approach for robustness testing," in IFIP international conference on testing of communicating systems. Springer, 2005, pp. 333-348.

[29] J. W. Baker, M. Schubert, and M. H. Faber, "On the assessment of robustness," Structural Safety, vol. 30, no. 3, pp. 253-267, 2008.

[30] H. Han, L.-T. J. Hsu, J.-S. Lee, and C. Sheu, "Are lodging customers ready to go green? an examination of attitudes, demographics, and ecofriendly intentions," International journal of hospitality management, vol. 30, no. 2, pp. 345-355, 2011.

[31] W. A. Asman, "Ammonia emission in europa: Updated emission and emission variations," RIVM Rapport 228471008, 1992.

[32] M.-L. Bourguet, "Designing and prototyping multimodal commands." in Interact, vol. 3. Citeseer, 2003, pp. 717-720.

[33] S. Kettebekov and R. Sharma, "Toward natural gesture/speech control of a large display," in IFIP International Conference on Engineering for Human-Computer Interaction. Springer, 2001, pp. 221-234.

[34] M. Gupta, M. Abdelsalam, S. Khorsandroo, and S. Mittal, "Security and privacy in smart farming: Challenges and opportunities," IEEE Access, vol. 8, pp. 34 564-34 584, 2020.

[35] I. Al Ridhawi, S. Otoum, M. Aloqaily, Y. Jararweh, and T. Baker, "Providing secure and reliable communication for next generation networks in smart cities," Sustainable Cities and Society, vol. 56, p. 102080, 2020. [Online]. Available: http://www.sciencedirect.com/ science/article/pii/S2210670720300676

[36] W. E. F. WEF. The impact of $5 \mathrm{~g}$ : Creating new value across industries and society. [Online]. Available: http://www3.weforum.org/ docs/WEF_The_Impact_of_5G_Report.pdf

[37] P. Ranaweera, A. D. Jurcut, and M. Liyanage, "Survey on multi-access edge computing security and privacy," IEEE Communications Surveys $\&$ Tutorials, pp. 1-1, 2021.

[38] T. Group. Building a 5g world we can all trust. [Online]. Available: https://www.thalesgroup.com/sites/default/files/ database/document/2020-12/tel-Trusted-5G_2.pdf

[39] I. T. Union. Itu ai/ml in $5 \mathrm{~g}$ challenge. [Online]. Available: https://www.itu.int/en/ITU-T/AI/challenge/2020/Pages/default.aspx

[40] P. Porambage, J. Okwuibe, M. Liyanage, M. Ylianttila, and T. Taleb, "Survey on multi-access edge computing for internet of things realization," IEEE Communications Surveys \& Tutorials, vol. 20, no. 4, pp. 2961-2991, 2018.

[41] B. Han, W. Jiang, M. A. Habibi, and H. D. Schotten, "An abstracted survey on 6g: Drivers, requirements, efforts, and enablers," 2021.

[42] H. Yu, M. K. Afzal, Y. B. Zikria, A. Rachedi, and F. H. Fitzek, "Tactile internet: Technologies, test platforms, trials, and applications," Future Generation Computer Systems, vol. 106, pp. 685-688, 2020. [Online]. Available: https://www.sciencedirect.com/science/article/pii/ S0167739X20304192

[43] I. I. T. Union. Report itu-r m.2370-0 : Imt traffic estimates for the years 2020 to 2030. [Online]. Available: https://www.itu.int/dms_pub/ itu-r/opb/rep/R-REP-M.2370-2015-PDF-E.pdf

[44] H. Tataria, M. Shafi, A. F. Molisch, M. Dohler, H. Sjöland, and F. Tufvesson, "6g wireless systems: Vision, requirements, challenges, insights, and opportunities," arXiv preprint arXiv:2008.03213, 2020.

[45] M. A. Habibi, M. Nasimi, B. Han, and H. D. Schotten, "A comprehensive survey of ran architectures toward $5 \mathrm{~g}$ mobile communication system," IEEE Access, vol. 7, pp. 70 371-70 421, 2019.

[46] R. Ali, Y. B. Zikria, A. K. Bashir, S. Garg, and H. S. Kim, "Urllc for $5 \mathrm{~g}$ and beyond: Requirements, enabling incumbent technologies and network intelligence," IEEE Access, pp. 1-1, 2021. 
[47] R. Khan, P. Kumar, D. N. K. Jayakody, and M. Liyanage, "A survey on security and privacy of $5 \mathrm{~g}$ technologies: Potential solutions, recent advancements, and future directions," IEEE Communications Surveys \& Tutorials, vol. 22, no. 1, pp. 196-248, 2020.

[48] S. E. Bibri, The IoT and Big Data Analytics for Smart Sustainable Cities: Enabling Technologies and Practical Applications. Cham: Springer International Publishing, 2020, pp. 191-226. [Online]. Available: https://doi.org/10.1007/978-3-030-41746-8_8

[49] F. Cappa, R. Oriani, E. Peruffo, and I. McCarthy, "Big data for creating and capturing value in the digitalized environment: Unpacking the effects of volume, variety, and veracity on firm performance," Journal of Product Innovation Management, vol. 38, no. 1, pp. 49-67, 2021.

[50] N. Shehab, M. Badawy, and H. Arafat, "Big data analytics and preprocessing," in Machine Learning and Big Data Analytics Paradigms: Analysis, Applications and Challenges. Springer, 2021, pp. 25-43.

[51] Y. Guo, B. Zhang, Y. Sun, K. Jiang, and K. Wu, "Machine learning based feature selection and knowledge reasoning for cbr system under big data," Pattern Recognition, vol. 112, p. 107805, 2021.

[52] G. Sharma, V. Tripathi, and A. Srivastava, "Recent trends in big data ingestion tools: A study," in Research in Intelligent and Computing in Engineering. Springer, 2021, pp. 873-881.

[53] B. Vamsi, B. P. Doppala, N. T. Rao, and D. Bhattacharyya, "Comparative analysis of prevalent disease by preprocessing techniques using big data and machine learning: An extensive review," Machine Intelligence and Soft Computing, pp. 27-38, 2021.

[54] Y. Hajjaji, W. Boulila, I. R. Farah, I. Romdhani, and A. Hussain, "Big data and iot-based applications in smart environments: A systematic review," Computer Science Review, vol. 39, p. 100318, 2021.

[55] D. Ardagna, C. Cappiello, W. Samá, and M. Vitali, "Context-aware data quality assessment for big data," Future Generation Computer Systems, vol. 89, pp. 548-562, 2018. [Online]. Available: https: //Www.sciencedirect.com/science/article/pii/S0167739X17329151

[56] S. Mazumdar, D. Seybold, K. Kritikos, and Y. Verginadis, "A survey on data storage and placement methodologies for cloud-big data ecosystem," Journal of Big Data, vol. 6, no. 1, pp. 1-37, 2019.

[57] A. Garg, N. Mittal et al., "A security and confidentiality survey in wireless internet of things (iot)," in Internet of Things and Big Data Applications. Springer, 2020, pp. 65-88.

[58] M. U. Khan, A. R. Javed, M. Ihsan, and U. Tariq, "A novel category detection of social media reviews in the restaurant industry," Multimedia Systems, pp. 1-14, 2020.

[59] B. Bellalta, L. Bononi, R. Bruno, and A. Kassler, "Next generation ieee 802.11 wireless local area networks: Current status, future directions and open challenges," Computer Communications, vol. 75 , pp. 1 - 25, 2016. [Online]. Available: http://www.sciencedirect.com/ science/article/pii/S0140366415003874

[60] Q. T. FEIBUSTech. Wi-fi 6 industry impact report. [Online]. Available: https://www.qualcomm.com/media/documents/ files/wi-fi-6-industry-impact-report.pdf

[61] E. Khorov, I. Levitsky, and I. F. Akyildiz, "Current status and directions of ieee 802.11 be, the future wi-fi7," IEEE Access, vol. 8, pp. 88664 88688,2020

[62] R. Ali, Y. B. Zikria, B.-S. Kim, and S. W. Kim, "Deep reinforcement learning paradigm for dense wireless networks in smart cities," in Smart cities performability, cognition, \& security. Springer, 2020, pp. 43-70.

[63] B. Chen, J. Wan, L. Shu, P. Li, M. Mukherjee, and B. Yin, "Smart factory of industry 4.0: Key technologies, application case, and challenges," IEEE Access, vol. 6, pp. 6505-6519, 2018.

[64] J.-P. A. Yaacoub, O. Salman, H. N. Noura, N. Kaaniche, A. Chehab, and M. Malli, "Cyber-physical systems security: Limitations, issues and future trends," Microprocessors and Microsystems, vol. 77, p. 103201, 2020.

[65] A. R. Javed, M. O. Beg, M. Asim, T. Baker, and A. H. Al-Bayatti, "Alphalogger: Detecting motion-based side-channel attack using smartphone keystrokes," Journal of Ambient Intelligence and Humanized Computing, pp. 1-14, 2020.

[66] S. Nahavandi, "Industry 5.0-a human-centric solution," Sustainability, vol. 11, no. 16, 2019. [Online]. Available: https://www.mdpi.com/ $2071-1050 / 11 / 16 / 4371$

[67] J. Vogt, "Where is the human got to go? artificial intelligence, machine learning, big data, digitalisation, and human-robot interaction in industry 4.0 and 5.0," 2021.

[68] X. Chen, M. A. Eder, A. Shihavuddin, and D. Zheng, "A humancyber-physical system toward intelligent wind turbine operation and maintenance," Sustainability, vol. 13, no. 2, 2021. [Online]. Available: https://www.mdpi.com/2071-1050/13/2/561
[69] A. R. Javed, M. U. Sarwar, S. Khan, C. Iwendi, M. Mittal, and N. Kumar, "Analyzing the effectiveness and contribution of each axis of tri-axial accelerometer sensor for accurate activity recognition," Sensors, vol. 20, no. 8, p. 2216, 2020.

[70] M. U. Sarwar and A. R. Javed, "Collaborative health care plan through crowdsource data using ambient application," in 2019 22nd International Multitopic Conference (INMIC). IEEE, 2019, pp. 1-6.

[71] W. Zehra, A. R. Javed, Z. Jalil, H. U. Khan, and T. R. Gadekallu, "Cross corpus multi-lingual speech emotion recognition using ensemble learning," Complex \& Intelligent Systems, pp. 1-10, 2021

[72] F. Aslam, W. Aimin, M. Li, and K. Ur Rehman, "Innovation in the era of iot and industry 5.0: absolute innovation management (aim) framework," Information, vol. 11, no. 2, p. 124, 2020.

[73] D. Kiran, I. Sharma, and I. Garg, "Industry 5.0 and smart cities: A futuristic approach," European Journal of Molecular \& Clinical Medicine, vol. 7, no. 8, pp. 2750-2756, 2020.

[74] M. Rosemann, J. Becker, and F. Chasin, "City 5.0," Business \& Information Systems Engineering, pp. 1-7, 2020

[75] M. Kowalkiewicz, M. Rosemann, and P. Dootson, Retail 5.0: Check-out the future. Australia: PwC Chair in Digital Economy, 2017. [Online]. Available: https://eprints.qut.edu.au/110377/

[76] M. Kowalkiewicz and P. Dootson, Government 5.0: the future of public services. Australia: The Chair in Digital Economy, 2019. [Online]. Available: https://eprints.qut.edu.au/133743/

[77] P. B. Tookanlou and H. Wong, "Determining the optimal customization levels, lead times, and inventory positioning in vertical product differentiation," International Journal of Production Economics, vol. 221, p 107479,2020

[78] O. A. ElFar, C.-K. Chang, H. Y. Leong, A. P. Peter, K. W. Chew, and P. L. Show, "Prospects of industry 5.0 in algae: Customization of production and new advance technology for clean bioenergy generation," Energy Conversion and Management: X, p. 100048, 2020.

[79] M. E. Studley and H. Little, "Robots in smart cities," in How Smart Is Your City? Springer, 2020, pp. 75-88.

[80] R. Macrorie, S. Marvin, and A. While, "Robotics and automation in the city: a research agenda," Urban Geography, vol. 0, no. 0, pp. 1-21, 2019. [Online]. Available: https://doi.org/10.1080/02723638. 2019.1698868

[81] K. M. Kumaran and M. Chinnadurai, "Cloud-based robotic system for crowd control in smart cities using hybrid intelligent generic algorithm," Journal of Ambient Intelligence and Humanized Computing, pp. $1-14,2020$.

[82] J. J. Roldán-Gómez, E. González-Gironda, and A. Barrientos, "A survey on robotic technologies for forest firefighting: Applying drone swarms to improve firefighters' efficiency and safety," Applied Sciences, vol. 11, no. 1, p. 363, 2021.

[83] M. Mamchenko, P. Ananyev, A. Kontsevoy, A. Plotnikova, and Y. Gromov, "The concept of robotics complex for transporting special equipment to emergency zones and evacuating wounded people," in Proceedings of 15th International Conference on Electromechanics and Robotics" Zavalishin's Readings". Springer, 2021, pp. 211-223.

[84] G. T. Gonzalez, U. Kaur, M. Rahman, V. Venkatesh, N. Sanchez, G. Hager, Y. Xue, R. Voyles, and J. Wachs, "From the dexterous surgical skill to the battlefield-a robotics exploratory study," Military Medicine, vol. 186, no. Supplement_1, pp. 288-294, 2021.

[85] K. R. Krishna, Aerial Robotics in Agriculture: Parafoils, Blimps, Aerostats, and Kites. CRC Press, 2021.

[86] K. Huang, D. Subedi, R. Mitra, I. Yung, K. Boyd, E. Aldrich, and D. Chitrakar, "Telelocomotion-remotely operated legged robots," Applied Sciences, vol. 11, no. 1, p. 194, 2021.

[87] A. Marchetti, C. Di Dio, F. Manzi, and D. Massaro, "Robotics in clinical and developmental psychology," Reference module in neuroscience and biobehavioral psychology, 2022.

[88] A. M. Qureshi, J. Lymer, and S. Dougherty, "Robotic small sat servicing: A next generation servicing architecture incorporating advanced robotics," in AIAA Scitech 2021 Forum, 2021, p. 0073.

[89] O. Zaki, M. Dunnigan, V. Robu, and D. Flynn, "Reliability and safety of autonomous systems based on semantic modelling for selfcertification. robotics 2021, 10, 10," 2021.

[90] D. Mitchell, O. Zaki, J. Blanche, J. Roe, L. Kong, S. Harper, V. Robu, T. Lim, and D. Flynn, "Symbiotic system design for safe and resilient autonomous robotics in offshore wind farms," arXiv preprint arXiv:2101.09491, 2021.

[91] T. Soyata, H. Habibzadeh, C. Ekenna, B. Nussbaum, and J. Lozano, "Smart city in crisis: Technology and policy concerns," Sustainable Cities and Society, vol. 50, p. 101566, 2019. 
[92] K. N. Qureshi, M. Q. Tayyab, S. U. Rehman, and G. Jeon, "An interference aware energy efficient data transmission approach for smart cities healthcare systems," Sustainable Cities and Society, vol. 62, p. 102392, 2020. [Online]. Available: http://www.sciencedirect. com/science/article/pii/S2210670720306132

[93] A. R. Javed, M. U. Sarwar, M. O. Beg, M. Asim, T. Baker, and H. Tawfik, "A collaborative healthcare framework for shared healthcare plan with ambient intelligence," Human-centric Computing and Information Sciences, vol. 10, no. 1, pp. 1-21, 2020.

[94] I. D. Corporation. Worldwide wearables market forecast to maintain double-digit growth in 2020 and through 2024, according to idc. [Online]. Available: https://www.idc.com/getdoc.jsp?containerId= prUS46885820

[95] G. Manogaran, R. Varatharajan, D. Lopez, P. M. Kumar, R. Sundarasekar, and C. Thota, "A new architecture of internet of things and big data ecosystem for secured smart healthcare monitoring and alerting system," Future Generation Computer Systems, vol. 82, pp. 375 - 387, 2018. [Online]. Available: http://www.sciencedirect.com/science/article/pii/S0167739X17305149

[96] B. R. Ecclestone, K. Bell, S. Abbasi, D. Dinakaran, M. Taher, J. R. Mackey, and P. H. Reza, "Histopathology for mohs micrographic surgery with photoacoustic remote sensing microscopy," Biomed. Opt. Express, vol. 12, no. 1, pp. 654-665, Jan 2021. [Online]. Available: http://www.osapublishing.org/boe/abstract.cfm?URI=boe-12-1-654

[97] R. Latifi, X. Da Dong, Z. Abouezzi, A. Kaul, A. Caine, R. Bergamaschi, A. Rojas, I. A. Laskowski, D. C. Koo, T. L. Weigel, K. Alizadeh, N. Gopal, A. Saji, A. Dixon, B. Zhang, J. Phillips, J. B. Cooper, and C. D. Gandhi, Surgical Telementoring and Teleproctoring. Cham: Springer International Publishing, 2021, pp. 431-453. [Online] Available: https://doi.org/10.1007/978-3-030-56917-4_27

[98] T. Yaqoob, H. Abbas, and M. Atiquzzaman, "Security vulnerabilities, attacks, countermeasures, and regulations of networked medical devices-a review," IEEE Communications Surveys Tutorials, vol. 21, no. 4, pp. 3723-3768, 2019

[99] J. Růžička and K. Navrátilová, "Crisis management as the part of smart traffic control in cities," in 2020 Smart City Symposium Prague (SCSP), 2020 , pp. $1-5$.

[100] F. Bénaben, M. Lauras, S. Truptil, and N. Salatgé, "A metamodel for knowledge management in crisis management," in 2016 49th Hawaii International Conference on System Sciences (HICSS), 2016, pp. 126135.

[101] C. Fan, C. Zhang, A. Yahja, and A. Mostafavi, "Disaster city digital twin: A vision for integrating artificial and human intelligence for disaster management," International Journal of Information Management, vol. 56, p. 102049, 2021.

[102] U. N. O. F. T. C. O. H. A. (OCHA). (2018) Ocha strategic plan, 2018-21. [Online]. Available: https://www.unocha.org/sites/unocha/ files/OCHA

[103] —. (2021) Ocha global humantarian overview 2021 - abridged version. [Online]. Available: https://www.unocha.org/sites/unocha/files/ GHO-2021-Abridged-EN.pdf

[104] S. Dong, A. Esmalian, H. Farahmand, and A. Mostafavi, "An integrated physical-social analysis of disrupted access to critical facilities and community service-loss tolerance in urban flooding," Computers, Environment and Urban Systems, 2020.

[105] L. Petersen, L. Fallou, P. Reilly, and E. Serafinelli, "Public expectations of critical infrastructure operators in times of crisis," Sustainable and Resilient Infrastructure, vol. 5, no. 1-2, pp. 62-77, 2020.

[106] J. Andharia, "Blurred boundaries, shared practices: Disaster studies as an emerging discipline and disaster management as a field of practice," Disaster Studies, pp. 33-76, 2020.

[107] S. U. Rehman, A. R. Javed, M. U. Khan, M. Nazar Awan, A. Farukh, and A. Hussien, "Personalisedcomfort: a personalised thermal comfort model to predict thermal sensation votes for smart building residents," Enterprise Information Systems, pp. 1-23, 2020.

[108] W. Li, C. Koo, T. Hong, J. Oh, S. H. Cha, and S. Wang, "A novel operation approach for the energy efficiency improvement of the hvac system in office spaces through real-time big data analytics," Renewable and Sustainable Energy Reviews, vol. 127, p. 109885, 2020.

[109] M. S. Mirnaghi and F. Haghighat, "Fault detection and diagnosis of large-scale hvac systems in buildings using data-driven methods: A comprehensive review," Energy and Buildings, p. 110492, 2020.

[110] L. Yu, Y. Sun, Z. Xu, C. Shen, D. Yue, T. Jiang, and X. Guan, "Multiagent deep reinforcement learning for hvac control in commercial buildings," IEEE Transactions on Smart Grid, vol. 12, no. 1, pp. 407419,2020
[111] J. García-Sanz-Calcedo, N. de Sousa Neves, and J. P. A. Fernandes, "Measurement of embodied carbon and energy of hvac facilities in healthcare centers," Journal of Cleaner Production, p. 125151, 2020.

[112] J. Latham, "The myth of a food crisis," in Rethinking Food and Agriculture. Elsevier, 2021, pp. 93-111.

[113] S. Singh, R. Kumar, R. Panchal, and M. K. Tiwari, "Impact of covid-19 on logistics systems and disruptions in food supply chain," International Journal of Production Research, pp. 1-16, 2020.

[114] A. L. Dahir, “'instead of coronavirus, the hunger will kill us.'a global food crisis looms," The New York Times, vol. 22, 2020

[115] L. Pafnoiu et al., "Food crisis-global priority," Ovidius University Annals, Economic Sciences Series, vol. 20, no. 1, pp. 232-236, 2020.

[116] Y. Liu, C. Yang, L. Jiang, S. Xie, and Y. Zhang, "Intelligent edge computing for iot-based energy management in smart cities," IEEE Network, vol. 33, no. 2, pp. 111-117, 2019.

[117] Z. Ullah, F. Al-Turjman, L. Mostarda, and R. Gagliardi, "Applications of artificial intelligence and machine learning in smart cities," Computer Communications, 2020.

[118] V. Fernandez-Anez, J. M. Fernández-Güell, and R. Giffinger, "Smart city implementation and discourses: An integrated conceptual model. the case of vienna," Cities, vol. 78, pp. 4 - 16, 2018 [Online]. Available: http://www.sciencedirect.com/science/article/pii/ S0264275117306558

[119] F. Pinna, F. Masala, and C. Garau, "Urban policies and mobility trends in italian smart cities," Sustainability, vol. 9, no. 4, p. 494, Mar 2017. [Online]. Available: http://dx.doi.org/10.3390/su9040494

[120] R. Cowley, S. Joss, and Y. Dayot, "The smart city and its publics: insights from across six uk cities," Urban Research \& Practice, vol. 11, no. 1, pp. 53-77, 2018. [Online]. Available: https://doi.org/10.1080/17535069.2017.1293150

[121] A. Datta, "The digital turn in postcolonial urbanism: Smart citizenship in the making of india's 100 smart cities," Transactions of the Institute of British Geographers, vol. 43, no. 3, pp. 405-419, 2018. [Online] Available: https://rgs-ibg.onlinelibrary.wiley.com/doi/abs/10.1111/tran. 12225

[122] A. Karvonen, F. Cugurullo, and F. Caprotti, Inside Smart Cities : Place, Politics and Urban Innovation. Taylor \& Francis Group, 2019, qC 20180416

[123] A.-M. Valdez, M. Cook, and S. Potter, "Roadmaps to utopia: Tales of the smart city," Urban Studies, vol. 55, no. 15, pp. 3385-3403, 2018 [Online]. Available: https://doi.org/10.1177/0042098017747857

[124] Y. Wu, W. Zhang, J. Shen, Z. Mo, and Y. Peng, "Smart city with chinese characteristics against the background of big data: Idea, action and risk," Journal of Cleaner Production, vol. 173, pp. 60 - 66, 2018, sustainable urban transformations towards smarter healthier cities: theories, agendas and pathways. [Online]. Available: http://www.sciencedirect.com/science/article/pii/S0959652617300549

[125] R. Dowling, P. McGuirk, and C. Gillon, "Strategic or piecemeal? smart city initiatives in sydney and melbourne," Urban Policy and Research, vol. 37, no. 4, pp. 429-441, 2019. [Online]. Available: https://doi.org/10.1080/08111146.2019.1674647

[126] S. Joss, F. Sengers, D. Schraven, F. Caprotti, and Y. Dayot, "The smart city as global discourse: Storylines and critical junctures across 27 cities," Journal of Urban Technology, vol. 26, no. 1, pp. 3-34, 2019. [Online]. Available: https://doi.org/10.1080/10630732.2018.1558387

[127] E. Alpaydin, Introduction to machine learning. MIT press, 2020.

[128] I. H. Sarker, "Machine learning: Algorithms, real-world applications and research directions," SN Computer Science, vol. 2, no. 3, pp. 1$21,2021$.

[129] M. Zekić-Sušac, S. Mitrović, and A. Has, "Machine learning based system for managing energy efficiency of public sector as an approach towards smart cities," International journal of information management, p. $102074,2020$.

[130] M. Shafiq, Z. Tian, A. K. Bashir, A. Jolfaei, and X. Yu, "Data mining and machine learning methods for sustainable smart cities traffic classification: A survey," Sustainable Cities and Society, vol. 60, p. 102177,2020

[131] D. Iskandaryan, F. Ramos, and S. Trilles, "Air quality prediction in smart cities using machine learning technologies based on sensor data: A review," Applied Sciences, vol. 10, no. 7, p. 2401, 2020.

[132] I. A. T. Hashem, A. E. Ezugwu, M. A. Al-Garadi, I. N. Abdullahi, O. Otegbeye, Q. O. Ahman, G. C. Mbah, A. K. Shukla, and H. Chiroma, "A machine learning solution framework for combatting covid-19 in smart cities from multiple dimensions," medRxiv, 2020.

[133] P. Kumar, G. P. Gupta, and R. Tripathi, "Tp2sf: A trustworthy privacypreserving secured framework for sustainable smart cities by leveraging 
blockchain and machine learning," Journal of Systems Architecture, p. 101954, 2020.

[134] S. M. H. Fard, H. Karimimpour, A. Dehghantanha, A. N. Jahromi, and G. Srivastava, "Ensemble sparse representation-based cyber threat hunting for security of smart cities," Computers \& Electrical Engineering, vol. 88 , p. $106825,2020$.

[135] H. Kim and J. Ben-Othman, "Toward integrated virtual emotion system with ai applicability for secure cps-enabled smart cities: Ai-based research challenges and security issues," IEEE Network, vol. 34, no. 3, pp. 30-36, 2020.

[136] M. M. Rashid, J. Kamruzzaman, M. M. Hassan, T. Imam, and S. Gordon, "Cyberattacks detection in iot-based smart city applications using machine learning techniques," International Journal of Environmental Research and Public Health, vol. 17, no. 24, p. 9347, 2020.

[137] M. A. Rahman, A. T. Asyhari, L. Leong, G. Satrya, M. H. Tao, and M. Zolkipli, "Scalable machine learning-based intrusion detection system for iot-enabled smart cities," Sustainable Cities and Society, vol. 61, p. 102324, 2020.

[138] A. Basit, M. Zafar, X. Liu, A. R. Javed, Z. Jalil, and K. Kifayat, "A comprehensive survey of ai-enabled phishing attacks detection techniques," Telecommunication Systems, pp. 1-16, 2020.

[139] Z. Allam, Big Data, Artificial Intelligence and the Rise of Autonomous Smart Cities. Cham: Springer International Publishing, 2021, pp. 730. [Online]. Available: https://doi.org/10.1007/978-3-030-59448-0_2

[140] F. O. Olowononi, D. B. Rawat, and C. Liu, "Resilient machine learning for networked cyber physical systems: A survey for machine learning security to securing machine learning for cps," IEEE Communications Surveys Tutorials, vol. 23, no. 1, pp. 524-552, 2021.

[141] A. Mohasseb, B. Aziz, J. Jung, and J. Lee, "Cyber security incidents analysis and classification in a case study of korean enterprises," Knowledge and Information Systems, vol. 62, no. 7, pp. 2917-2935, 2020.

[142] S. I. Imtiaz, S. ur Rehman, A. R. Javed, Z. Jalil, X. Liu, and W. S. Alnumay, "Deepamd: Detection and identification of android malware using high-efficient deep artificial neural network," Future Generation Computer Systems, vol. 115, pp. 844-856, 2020.

[143] M. A. Wani, F. A. Bhat, S. Afzal, and A. I. Khan, Advances in deep learning. Springer, 2020

[144] A. A. Elsaeidy, N. Jagannath, A. G. Sanchis, A. Jamalipour, and K. S. Munasinghe, "Replay attack detection in smart cities using deep learning," IEEE Access, vol. 8, pp. 137 825-137 837, 2020.

[145] S. K. Singh, Y.-S. Jeong, and J. H. Park, "A deep learning-based iotoriented infrastructure for secure smart city," Sustainable Cities and Society, vol. 60, p. 102252, 2020.

[146] D. Chen, P. Wawrzynski, and Z. Lv, "Cyber security in smart cities: A review of deep learning-based applications and case studies," Sustainable Cities and Society, p. 102655, 2020.

[147] R. Vinayakumar, M. Alazab, S. Srinivasan, Q.-V. Pham, S. K. Padannayil, and K. Simran, "A visualized botnet detection system based deep learning for the internet of things networks of smart cities," IEEE Transactions on Industry Applications, vol. 56, no. 4, pp. 4436-4456, 2020.

[148] M. A. Ferrag, L. Maglaras, S. Moschoyiannis, and H. Janicke, "Deep learning for cyber security intrusion detection: Approaches, datasets, and comparative study," Journal of Information Security and Applications, vol. 50, p. 102419, 2020.

[149] N. Magaia, R. Fonseca, K. Muhammad, A. H. F. N. Segundo, A. V. L. Neto, and V. H. C. de Albuquerque, "Industrial internet of things security enhanced with deep learning approaches for smart cities," IEEE Internet of Things Journal, 2020.

[150] A. R. Javed, M. Usman, S. U. Rehman, M. U. Khan, and M. S. Haghighi, "Anomaly detection in automated vehicles using multistage attention-based convolutional neural network," IEEE Transactions on Intelligent Transportation Systems, 2020.

[151] S. Afzal, M. Asim, A. R. Javed, M. O. Beg, and T. Baker, "Urldeepdetect: A deep learning approach for detecting malicious urls using semantic vector models," Journal of Network and Systems Management, vol. 29, no. 3, pp. 1-27, 2021.

[152] M. Aqib, R. Mehmood, A. Alzahrani, and I. Katib, "A smart disaster management system for future cities using deep learning, gpus, and in-memory computing," in Smart Infrastructure and Applications. Springer, 2020, pp. 159-184.

[153] B. Ghose and Z. Rehena, "A deep learning approach for predicting air pollution in smart cities," in Computational Intelligence and Machine Learning. Springer, 2021, pp. 29-38.

[154] S. C. K. Tekouabou, W. Cherif, H. Silkan et al., "Improving parking availability prediction in smart cities with iot and ensemble-based model," Journal of King Saud University-Computer and Information Sciences, 2020.

[155] Y. Chen, X. Zou, K. Li, K. Li, X. Yang, and C. Chen, "Multiple local 3d cnns for region-based prediction in smart cities," Information Sciences, vol. 542, pp. 476-491, 2021.

[156] A. Belhadi, Y. Djenouri, G. Srivastava, D. Djenouri, J. C.-W. Lin, and G. Fortino, "Deep learning for pedestrian collective behavior analysis in smart cities: A model of group trajectory outlier detection," Information Fusion, vol. 65, pp. 13-20, 2021.

[157] S. Khan, S. Nazir, and H. Anwar, "Deep learning-based urban big data fusion in smart cities: Towards traffic monitoring and flow-preserving fusion," Computers \& Electrical Engineering, vol. 89, p. 106906, 2021.

[158] A. Alghamdi, M. Hammad, H. Ugail, A. Abdel-Raheem, K. Muhammad, H. S. Khalifa, and A. A. Abd El-Latif, "Detection of myocardial infarction based on novel deep transfer learning methods for urban healthcare in smart cities," Multimedia tools and applications, pp. 122,2020

[159] M. A. Al-Garadi, A. Mohamed, A. K. Al-Ali, X. Du, I. Ali, and M. Guizani, "A survey of machine and deep learning methods for internet of things (iot) security," IEEE Communications Surveys Tutorials, vol. 22, no. 3, pp. 1646-1685, 2020.

[160] N. C. Luong, D. T. Hoang, S. Gong, D. Niyato, P. Wang, Y. Liang, and D. I. Kim, "Applications of deep reinforcement learning in communications and networking: A survey," IEEE Communications Surveys Tutorials, vol. 21, no. 4, pp. 3133-3174, 2019.

[161] Y. B. Zikria, M. K. Afzal, S. W. Kim, A. Marin, and M. Guizani, "Deep learning for intelligent iot: Opportunities, challenges and solutions," Computer Communications, vol. 164, pp. 50-53, 2020. [Online]. Available: https://www.sciencedirect.com/science/article/pii/ S0140366420319046

[162] A. R. Javed and Z. Jalil, "Byte-level object identification for forensic investigation of digital images," in 2020 International Conference on Cyber Warfare and Security (ICCWS). IEEE, 2020, pp. 1-4.

[163] S. Gupta and B. Sundar, "A computer vision based approach for automated traffic management as a smart city solution," in 2020 IEEE International Conference on Electronics, Computing and Communication Technologies (CONECCT), 2020, pp. 1-6.

[164] M.-C. Chang, C.-K. Chiang, C.-M. Tsai, Y.-K. Chang, H.-L. Chiang, Y.-A. Wang, S.-Y. Chang, Y.-L. Li, M.-S. Tsai, and H.-Y. Tseng, "Ai city challenge 2020 - computer vision for smart transportation applications," in Proceedings of the IEEE/CVF Conference on Computer Vision and Pattern Recognition (CVPR) Workshops, June 2020.

[165] J. G. Shanahan and L. Dai, "Introduction to computer vision and real time deep learning-based object detection," in Proceedings of the 26th ACM SIGKDD International Conference on Knowledge Discovery \& Data Mining, ser. KDD '20. New York, NY, USA: Association for Computing Machinery, 2020, p. 3523-3524. [Online]. Available: https://doi.org/10.1145/3394486.3406713

[166] S. Bhattacharya, S. R. K. Somayaji, T. R. Gadekallu, M. Alazab, and P. K. R. Maddikunta, "A review on deep learning for future smart cities," Internet Technology Letters, vol. n/a, no. n/a, p. e187, 2020. [Online]. Available: https://onlinelibrary.wiley.com/doi/abs/10. $1002 /$ itl2.187

[167] M. Shorfuzzaman, M. S. Hossain, and M. F. Alhamid, "Towards the sustainable development of smart cities through mass video surveillance: A response to the covid-19 pandemic," Sustainable cities and society, vol. 64, p. 102582, 2021.

[168] A. R. Neto, T. P. Silva, T. V. Batista, F. C. Delicato, P. F. Pires, and F. Lopes, "An architecture for distributed video stream processing in iomt systems," Open Journal of Internet Of Things (OJIOT), vol. 6 no. 1 , pp. 89-104, 2020.

[169] A. Rocha Neto, T. P. Silva, T. Batista, F. C. Delicato, P. F. Pires, and F. Lopes, "Leveraging edge intelligence for video analytics in smart city applications," Information, vol. 12, no. 1, p. 14, 2021.

[170] P. W. Tien, S. Wei, and J. Calautit, "A computer vision-based occupancy and equipment usage detection approach for reducing building energy demand," Energies, vol. 14, no. 1, p. 156, 2021.

[171] G. Laudante, V. Musone, M. Rak, S. Venticinque, and G. Salzillo, "A cloud-edge smart infrastructures for road safety," in 2020 IEEE 20th Mediterranean Electrotechnical Conference ( MELECON), 2020, pp. $147-152$.

[172] A. e. a. Finogeev, "Intelligent monitoring system for smart road environment," Journal of Industrial Information Integration, vol. 15, pp. $15-20,2019$

[173] A. R. Javed, R. Abid, B. Aslam, H. A. Khalid, M. Z. Khan, O. H. Alhazmi, and M. Rizwan, "Green5g: Enhancing capacity and coverage in device-to-device communication," CMC, 2021. 
[174] N. Chen, M. Wang, N. Zhang, and X. Shen, "Energy and information management of electric vehicular network: A survey," IEEE Communications Surveys Tutorials, vol. 22, no. 2, pp. 967-997, 2020.

[175] A. Rehman Javed, Z. Jalil, S. Atif Moqurrab, S. Abbas, and X. Liu, "Ensemble adaboost classifier for accurate and fast detection of botnet attacks in connected vehicles," Transactions on Emerging Telecommunications Technologies, vol. n/a, no. n/a, p. e4088, 2020. [Online]. Available: https://onlinelibrary.wiley.com/doi/abs/10.1002/ett. 4088

[176] S. Gyawali, S. Xu, Y. Qian, and R. Q. Hu, "Challenges and solutions for cellular based v2x communications," IEEE Communications Surveys \& Tutorials, vol. 23 , no. 1 , pp. 222-255, 2021

[177] J. Yan, J. Liu, and F.-M. Tseng, "An evaluation system based on the self-organizing system framework of smart cities: A case study of smart transportation systems in china," Technological Forecasting and Social Change, vol. 153, p. 119371, 2020. [Online]. Available: http://www.sciencedirect.com/science/article/pii/S0040162518301021

[178] G. Bakioglu and A. O. Atahan, "Ahp integrated topsis and vikor methods with pythagorean fuzzy sets to prioritize risks in self-driving vehicles," Applied Soft Computing, vol. 99, p. 106948, 2021. [Online]. Available: https://www.sciencedirect.com/science/article/pii/ S1568494620308863

[179] R. Hussain and S. Zeadally, "Autonomous cars: Research results, issues, and future challenges," IEEE Communications Surveys Tutorials, vol. 21, no. 2, pp. 1275-1313, 2019.

[180] I.-S. Sorlei, N. Bizon, P. Thounthong, M. Varlam, E. Carcadea, M. Culcer, M. Iliescu, and M. Raceanu, "Fuel cell electric vehicles-a brief review of current topologies and energy management strategies," Energies, vol. 14, no. 1, p. 252, 2021.

[181] A. Olabi, T. Wilberforce, and M. A. Abdelkareem, "Fuel cell application in the automotive industry and future perspective," Energy, vol. 214 , p. 118955,2021

[182] S. S. Ahmed, K. F. Hulme, G. Fountas, U. Eker, I. V. Benedyk, S. E. Still, and P. C. Anastasopoulos, "The flying car-challenges and strategies toward future adoption," Frontiers in Built Environment, vol. 6, p. 106, 2020. [Online]. Available: https://www.frontiersin.org/article/10.3389/fbuil.2020.00106

[183] S. S. Ahmed, G. Fountas, U. Eker, S. E. Still, and P. C. Anastasopoulos, "An exploratory empirical analysis of willingness to hire and pay for flying taxis and shared flying car services," Journal of Air Transport Management, vol. 90, p. 101963, 2021. [Online]. Available: http://www.sciencedirect.com/science/article/pii/S0969699720305469

[184] X. Liu, K. Reddi, A. Elgowainy, H. Lohse-Busch, M. Wang, and N. Rustagi, "Comparison of well-to-wheels energy use and emissions of a hydrogen fuel cell electric vehicle relative to a conventional gasoline-powered internal combustion engine vehicle," International Journal of Hydrogen Energy, vol. 45, no. 1, pp. 972-983, 2020.

[185] I. E. A. (IEA). Global demand for pure hydrogen, 19752018. [Online]. Available: https://www.iea.org/data-and-statistics/ charts/global-demand-for-pure-hydrogen-1975-2018

[186] A. Ferrara, S. Jakubek, and C. Hametner, "Energy management of heavy-duty fuel cell vehicles in real-world driving scenarios: Robust design of strategies to maximize the hydrogen economy and system lifetime," Energy Conversion and Management, p. 113795, 2021.

[187] A. Mofolasayo, "Potential policy issues with flying car technology," Transportation Research Procedia, vol. 48, pp. 8 - 22, 2020, recent Advances and Emerging Issues in Transport Research - An Editorial Note for the Selected Proceedings of WCTR 2019 Mumbai. [Online]. Available: http://www.sciencedirect.com/science/article/pii/ S2352146520304130

[188] M. N. Postorino and G. M. L. Sarné, "Reinventing mobility paradigms: Flying car scenarios and challenges for urban mobility," Sustainability, vol. 12, no. 9, p. 3581, Apr 2020. [Online]. Available: http://dx.doi.org/10.3390/su12093581

[189] C. O. A. W. C. Jeff Wilke. A drone program taking flight. [Online]. Available: https://www.aboutamazon.com/news/ transportation/a-drone-program-taking-flight

[190] H. Wang, H. Zhao, J. Zhang, D. Ma, J. Li, and J. Wei, "Survey on unmanned aerial vehicle networks: A cyber physical system perspective," IEEE Communications Surveys Tutorials, vol. 22, no. 2, pp. 10271070, 2020.

[191] S. A. Chaudhry, K. Yahya, M. Karuppiah, R. Kharel, A. K. Bashir, and Y. B. Zikria, "Gcacs-iod: A certificate based generic access control scheme for internet of drones," Computer Networks, vol. 191, p. 107999, 2021. [Online]. Available: https://www.sciencedirect.com/ science/article/pii/S1389128621001195
[192] P. Sanjeevikumar, "Green energy: Solar energy, photovoltaics, and smart cities," Wiley, 2021

[193] J. B. Park, R. J. Craggs, and C. C. Tanner, "Eco-friendly and lowcost enhanced pond and wetland (epw) system for the treatment of secondary wastewater effluent," Ecological Engineering, vol. 120, pp. 170-179, 2018.

[194] A. Iqbal and K. A. Al-Ghamdi, "Energy-efficient cellular manufacturing system: Eco-friendly revamping of machine shop configuration," Energy, vol. 163, pp. 863-872, 2018.

[195] S. Banjo, B. Bolaji, I. Osagie, O. Fayomi, O. Fakehinde, P. Olayiwola, S. Oyedepo, and N. Udoye, "Experimental analysis of the performance characteristic of an eco-friendly hc600a as a retrofitting refrigerant in a thermal system," in Journal of Physics: Conference Series, vol. 1378 , no. 4. IOP Publishing, 2019, p. 042033.

[196] M. Topaloglu, F. Yarkin, and T. Kaya, "Solid waste collection system selection for smart cities based on a type-2 fuzzy multi-criteria decision technique," Soft Computing, vol. 22, no. 15, pp. 4879-4890, 2018.

[197] T. Issac, S. Silas, and E. B. Rajsingh, "Dynamic and static system modeling with simulation of an eco-friendly smart lighting system," in Systems Simulation and Modeling for Cloud Computing and Big Data Applications. Elsevier, 2020, pp. 81-97.

[198] S. Urooj, F. Alrowais, Y. Teekaraman, H. Manoharan, and R. Kuppusamy, "Iot based electric vehicle application using boosting algorithm for smart cities," Energies, vol. 14, no. 4, p. 1072, 2021.

[199] M. Aloqaily, S. Otoum, I. Al Ridhawi, and Y. Jararweh, "An intrusion detection system for connected vehicles in smart cities," Ad Hoc Networks, vol. 90, p. 101842, 2019.

[200] I. Mahmood and J. A. Zubairi, "Efficient waste transportation and recycling: Enabling technologies for smart cities using the internet of things," IEEE Electrification Magazine, vol. 7, no. 3, pp. 33-43, 2019.

[201] T. Ali, M. Irfan, A. S. Alwadie, and A. Glowacz, "Iot-based smart waste bin monitoring and municipal solid waste management system for smart cities," Arabian Journal for Science and Engineering, vol. 45, pp. $10185-10198,2020$.

[202] J. Palanca, J. Jordán, J. Bajo, and V. Botti, "An energy-aware algorithm for electric vehicle infrastructures in smart cities," Future Generation Computer Systems, vol. 108, pp. 454-466, 2020

[203] W. Ejaz and A. Anpalagan, "Internet of things enabled electric vehicles in smart cities," in Internet of things for smart cities. Springer, 2019, pp. $39-46$.

[204] M. Laroui, A. Dridi, H. Afifi, H. Moungla, M. Marot, and M. A. Cherif, "Energy management for electric vehicles in smart cities: a deep learning approach," in 2019 15th International Wireless Communications \& Mobile Computing Conference (IWCMC). IEEE, 2019, pp. 20802085.

[205] B. Hu, Y. Feng, J. Sun, Y. Gao, and J. Tan, "Driving preference analysis and electricity pricing strategy comparison for electric vehicles in smart city," Information Sciences, vol. 504, pp. 202-220, 2019.

[206] M. S. Chaudhari, B. Patil, and V. Raut, "Iot based waste collection management system for smart cities: an overview," in 2019 3rd International Conference on Computing Methodologies and Communication (ICCMC). IEEE, 2019, pp. 802-805.

[207] M. I. Aceleanu, A. C. Serban, M.-C. Suciu, and T. I. Biţoiu, "The management of municipal waste through circular economy in the context of smart cities development," IEEE Access, vol. 7, pp. 133602 133614,2019

[208] M. Sharma, S. Joshi, D. Kannan, K. Govindan, R. Singh, and H. Purohit, "Internet of things (iot) adoption barriers of smart cities' waste management: An indian context," Journal of Cleaner Production, vol. 270 , p. 122047,2020

[209] F. Alqahtani, Z. Al-Makhadmeh, A. Tolba, and W. Said, "Internet of things-based urban waste management system for smart cities using a cuckoo search algorithm," Cluster Computing, vol. 23, pp. 1769-1780, 2020.

[210] S. Idwan, I. Mahmood, J. A. Zubairi, and I. Matar, "Optimal management of solid waste in smart cities using internet of things," Wireless Personal Communications, vol. 110, no. 1, pp. 485-501, 2020.

[211] Z. Mingaleva, N. Vukovic, I. Volkova, and T. Salimova, "Waste management in green and smart cities: A case study of russia," Sustainability, vol. 12, no. 1, p. 94, 2020.

[212] S. Srinivasan, V. Ravi, M. Alazab, S. Ketha, A.-Z. Ala'M, and S. K. Padannayil, "Spam emails detection based on distributed word embedding with deep learning," in Machine Intelligence and Big Data Analytics for Cybersecurity Applications. Springer, 2021, pp. 161189.

[213] A. Tandon, A. Dhir, A. N. Islam, and M. Mäntymäki, "Blockchain in healthcare: A systematic literature review, synthesizing framework 
and future research agenda," Computers in Industry, vol. 122, p. 103290, 2020. [Online]. Available: http://www.sciencedirect.com/ science/article/pii/S0166361520305248

[214] R. A. Popa, C. M. S. Redfield, N. Zeldovich, and H. Balakrishnan, "Cryptdb: Protecting confidentiality with encrypted query processing," in Proceedings of the Twenty-Third ACM Symposium on Operating Systems Principles, ser. SOSP '11. New York, NY, USA: Association for Computing Machinery, 2011, p. 85-100. [Online]. Available: https://doi.org/10.1145/2043556.2043566

[215] F. Shahzad, W. Iqbal, and F. S. Bokhari, "On the use of cryptdb for securing electronic health data in the cloud: A performance study," in 2015 17th International Conference on E-health Networking, Application Services (HealthCom), 2015, pp. 120-125.

[216] R. A. Mishra, A. Kalla, A. Braeken, and M. Liyanage, "Privacy protected blockchain based architecture and implementation for sharing of students' credentials," Information Processing \& Management vol. 58, no. 3, p. 102512, 2021. [Online]. Available: https: //www.sciencedirect.com/science/article/pii/S0306457321000212

[217] R. Kitchin and M. Dodge, "The (in)security of smart cities: Vulnerabilities, risks, mitigation, and prevention," Journal of Urban Technology, vol. 26, no. 2, pp. 47-65, 2019. [Online]. Available: https://doi.org/10.1080/10630732.2017.1408002

[218] Z. A. Baig, P. Szewczyk, C. Valli, P. Rabadia, P. Hannay, M. Chernyshev, M. Johnstone, P. Kerai, A. Ibrahim, K. Sansurooah, N. Syed, and M. Peacock, "Future challenges for smart cities: Cyber-security and digital forensics," Digital Investigation, vol. 22, pp. 3-13, 2017. [Online]. Available: https://www.sciencedirect.com/ science/article/pii/S1742287617300579

[219] M. M. Losavio, K. P. Chow, A. Koltay, and J. James, "The internet of things and the smart city: Legal challenges with digital forensics, privacy, and security," Security and Privacy, vol. 1, no. 3, p. e23, 2018. [Online]. Available: https://onlinelibrary.wiley.com/doi/abs/10. $1002 /$ spy 2.23

[220] W. Ahmed, F. Shahzad, A. R. Javed, F. Iqbal, and L. Ali, "Whatsapp network forensics: Discovering the ip addresses of suspects," in 2021 11th IFIP International Conference on New Technologies, Mobility and Security (NTMS), 2021, pp. 1-7.

[221] M. Li, C. Lal, M. Conti, and D. Hu, "Lechain: A blockchainbased lawful evidence management scheme for digital forensics," Future Generation Computer Systems, vol. 115, pp. 406-420, 2021. [Online]. Available: https://www.sciencedirect.com/science/article/pii/ S0167739X1933167X

[222] S. Mrdovic, IoT Forensics. Cham: Springer International Publishing, 2021, pp. 215-229. [Online]. Available: https://doi.org/10.1007/ 978-3-030-10591-4_13

[223] C. Arumugam and S. Shunmuganathan, "Digital forensics: Essential competencies of cyber-forensics practitioners," in Advances in Machine Learning and Computational Intelligence. Springer, 2021, pp. 843851.

[224] H. van Beek, J. van den Bos, A. Boztas, E. van Eijk, R. Schramp, and M. Ugen, "Digital forensics as a service: Stepping up the game," Forensic Science International: Digital Investigation, vol. 35, p. 301021, 2020. [Online]. Available: https://www.sciencedirect.com/ science/article/pii/S2666281720300706

[225] M. Stoyanova, Y. Nikoloudakis, S. Panagiotakis, E. Pallis, and E. K. Markakis, "A survey on the internet of things (iot) forensics: Challenges, approaches, and open issues," IEEE Communications Surveys Tutorials, vol. 22, no. 2, pp. 1191-1221, 2020.

[226] M. Mittal, C. Iwendi, S. Khan, and A. Rehman Javed, "Analysis of security and energy efficiency for shortest route discovery in low-energy adaptive clustering hierarchy protocol using levenberg-marquardt neural network and gated recurrent unit for intrusion detection system," Transactions on Emerging Telecommunications Technologies, $p$. e3997, 2020.

[227] M. Usman Sarwar, A. Rehman Javed, F. Kulsoom, S. Khan, U. Tariq, and A. Kashif Bashir, "Parciv: Recognizing physical activities having complex interclass variations using semantic data of smartphone," Software: Practice and Experience, vol. 51, no. 3, pp. 532-549, 2021

[228] D. Li, L. Deng, M. Lee, and H. Wang, "Iot data feature extraction and intrusion detection system for smart cities based on deep migration learning," International journal of information management, vol. 49, pp. $533-545,2019$.

[229] E. Ismagilova, L. Hughes, N. P. Rana, and Y. K. Dwivedi, "Security, privacy and risks within smart cities: Literature review and development of a smart city interaction framework," Information Systems Frontiers, pp. 1-22, 2020.
[230] Y. Hu, A. Yang, H. Li, S. Yuyan, and L. Sun, "On smart city and safe city concepts : A survey of intrusion detection on industrial control systems," International Journal of Distributed Sensor Networks, vol. 14, no. 8, pp. 836-845, 2018. [Online]. Available: https://journals.sagepub.com/doi/abs/10.1177/1550147718794615

[231] F. Al-Turjman, "Intelligence and security in big 5g-oriented iont: An overview," Future Generation Computer Systems, vol. 102, pp. 357 368, 2020. [Online]. Available: http://www.sciencedirect.com/science/ article/pii/S0167739X19301074

[232] C. Iwendi, Z. Jalil, A. R. Javed, T. Reddy, R. Kaluri, G. Srivastava, and O. Jo, "Keysplitwatermark: Zero watermarking algorithm for software protection against cyber-attacks," IEEE Access, vol. 8, pp. 72650$72660,2020$.

[233] S. ur Rehman, M. Khaliq, S. I. Imtiaz, A. Rasool, M. Shafiq, A. R. Javed, Z. Jalil, and A. K. Bashir, "Diddos: An approach for detection and identification of distributed denial of service (ddos) cyberattacks using gated recurrent units (gru)," Future Generation Computer Systems, vol. 118, pp. 453-466, 2021.

[234] A. Rehman, S. U. Rehman, M. Khan, M. Alazab, and T. Reddy, "Canintelliids: Detecting in-vehicle intrusion attacks on a controller area network using cnn and attention-based gru," IEEE Transactions on Network Science and Engineering, 2021.

[235] A. Basit, M. Zafar, A. R. Javed, and Z. Jalil, "A novel ensemble machine learning method to detect phishing attack," in 2020 IEEE 23rd International Multitopic Conference (INMIC). IEEE, 2020, pp. $1-5$.

[236] V. Ravi, M. Alazab, S. Srinivasan, A. Arunachalam, and K. Soman, "Adversarial defense: Dga-based botnets and dns homographs detection through integrated deep learning," IEEE Transactions on Engineering Management, 2021.

[237] S. Sriram, R. Vinayakumar, M. Alazab, and K. Soman, "Network flow based iot botnet attack detection using deep learning," in IEEE INFOCOM 2020-IEEE Conference on Computer Communications Workshops (INFOCOM WKSHPS). IEEE, 2020, pp. 189-194.

[238] L. Yang, N. Elisa, and N. Eliot, "Privacy and security aspects of egovernment in smart cities," in Smart cities cybersecurity and privacy. Elsevier, 2019, pp. 89-102.

[239] R. A. Ramadan, "Efficient intrusion detection algorithms for smart cities-based wireless sensing technologies," Journal of Sensor and Actuator Networks, vol. 9, no. 3, p. 39, 2020.

[240] N. Mohammad, "A multi-tiered defense model for the security analysis of critical facilities in smart cities," IEEE Access, vol. 7, pp. 152585 $152598,2019$.

[241] A. Aldaej, "Enhancing cyber security in modern internet of things (iot) using intrusion prevention algorithm for iot (ipai)," IEEE Access, 2019.

[242] K. N. Qureshi, S. S. Rana, A. Ahmed, and G. Jeon, "A novel and secure attacks detection framework for smart cities industrial internet of things," Sustainable Cities and Society, vol. 61, p. 102343, 2020.

[243] N. Chaabouni, M. Mosbah, A. Zemmari, C. Sauvignac, and P. Faruki, "Network intrusion detection for iot security based on learning techniques," IEEE Communications Surveys Tutorials, vol. 21, no. 3, pp. $2671-2701,2019$.

[244] M. Rana, A. Shafiq, I. Altaf, M. Alazab, K. Mahmood, S. A. Chaudhry, and Y. B. Zikria, "A secure and lightweight authentication scheme for next generation iot infrastructure," Computer Communications, vol. 165, pp. 85-96, 2021. [Online]. Available: https://www.sciencedirect. com/science/article/pii/S0140366420319745

[245] J. Niemann and A. Pisla, Smart Life Cycle Services. Cham: Springer International Publishing, 2021, pp. 107-121. [Online]. Available: https://doi.org/10.1007/978-3-030-56449-0_9

[246] M. J. Kim and H. J. Jun, "Towards a sustainable life: Smart and green design in buildings and community," Sustainability, vol. 13, no. 3, 2021. [Online]. Available: https://www.mdpi.com/2071-1050/ $13 / 3 / 1022$

[247] D. Srivastava, S. Rakesh kumar, N. Gayathri, and F. Al-Turjman, "Chapter 10 - security aspects and uavs in socialized regions," in Security in IoT Social Networks, ser. Intelligent Data-Centric Systems, F. Al-Turjman and B. Deebak, Eds. Academic Press, 2021, pp. 229-245. [Online]. Available: https://www.sciencedirect.com/science/ article/pii/B9780128215999000108

[248] D. Orosz, "Examining the contribution of smart homes to the smart performance of cities," THEORY METHODOLOGY PRACTICE: CLUB OF ECONOMICS IN MISKOLC, vol. 17, no. SI, pp. 23-30, 2021.

[249] K. D., A. Harshavardhan, V. Manoj Kumar, D. Sunitha, and S. N. Korra, "Ble in iot: Improved link stability and energy conservation using fuzzy approach for smart homes automation," 
Materials Today: Proceedings, 2021. [Online]. Available: https: //www.sciencedirect.com/science/article/pii/S221478532040094X

[250] J. Wang, N. Spicher, J. M. Warnecke, M. Haghi, J. Schwartze, and T. M. Deserno, "Unobtrusive health monitoring in private spaces: The smart home," Sensors, vol. 21, no. 3, 2021. [Online]. Available: https://www.mdpi.com/1424-8220/21/3/864

[251] S. Oueida, M. Aloqaily, and S. Ionescu, "A smart healthcare reward model for resource allocation in smart city," Multimedia tools and applications, vol. 78, no. 17, pp. 24 573-24 594, 2019.

[252] B. Xu, L. Li, D. Hu, B. Wu, C. Ye, and H. Cai, "Healthcare data analysis system for regional medical union in smart city," Journal of Management Analytics, vol. 5, no. 4, pp. 334-349, 2018. [Online]. Available: https://doi.org/10.1080/23270012.2018.1490211

[253] N. Gavrilović and A. Mishra, "Software architecture of the internet of things (iot) for smart city, healthcare and agriculture: analysis and improvement directions," Journal of Ambient Intelligence and Humanized Computing, pp. 1-22, 2020.

[254] J. A. Malek, S. B. Lim, and T. Yigitcanlar, "Social inclusion indicators for building citizen-centric smart cities: A systematic literature review," Sustainability, vol. 13, no. 1, 2021. [Online]. Available: https://www.mdpi.com/2071-1050/13/1/376

[255] T. Shafeer, M. Senthil Arumugam, and A. Sasikala, "On the design and applications of an integrated smart home automation," in Data Intelligence and Cognitive Informatics, I. Jeena Jacob, S. Kolandapalayam Shanmugam, S. Piramuthu, and P. Falkowski-Gilski, Eds. Singapore: Springer Singapore, 2021, pp. 35-52.

[256] S. K. H. M. Siddiqa, K. Apurva, D. Nandan, and S. Kumar, "Documentation on smart home monitoring using internet of things," in ICCCE 2020, A. Kumar and S. Mozar, Eds. Singapore: Springer Singapore, 2021 , pp. $1115-1124$.

[257] S. F. Tahir, L. G. Fahad, and K. Kifayat, "Key feature identification for recognition of activities performed by a smart-home resident," Journal of Ambient Intelligence and Humanized Computing, vol. 11, no. 5, pp. $2105-2115,2020$

[258] A. R. Javed, L. G. Fahad, A. A. Farhan, S. Abbas, G. Srivastava, R. M Parizi, and M. S. Khan, "Automated cognitive health assessment in smart homes using machine learning," Sustainable Cities and Society, vol. 65 , p. 102572, 2021.

[259] M. Shabbir, A. Shabbir, C. Iwendi, A. R. Javed, M. Rizwan, N. Herencsar, and J. C.-W. Lin, "Enhancing security of health information using modular encryption standard in mobile cloud computing," IEEE Access, vol. 9, pp. 8820-8834, 2021.

[260] T. M. Vinod Kumar and B. Dahiya, Smart Economy in Smart Cities. Singapore: Springer Singapore, 2017, pp. 3-76. [Online]. Available: https://doi.org/10.1007/978-981-10-1610-3_1

[261] P. Cardullo and R. Kitchin, "Being a "citizen'in the smart city: up an down the scaffold of smart citizen participation in dublin, ireland," GeoJournal, vol. 84, no. 1, pp. 1-13, 2019.

[262] A. Simonofski, T. Vallé, E. Serral, and Y. Wautelet, "Investigating context factors in citizen participation strategies: A comparative analysis of swedish and belgian smart cities," International Journal of Information Management, vol. 56, p. 102011, 2021. [Online]. Available: https://www.sciencedirect.com/science/article/pii/S0268401219302439

[263] M. J. Nikki Han and M. J. Kim, "A critical review of the smart city in relation to citizen adoption towards sustainable smart living," Habitat International, vol. 108, p. 102312, 2021. [Online]. Available: https://www.sciencedirect.com/science/article/pii/S0197397521000011

[264] T. Ji, J.-H. Chen, H.-H. Wei, and Y.-C. Su, "Towards peoplecentric smart city development: Investigating the citizens' preferences and perceptions about smart-city services in taiwan," Sustainable Cities and Society, vol. 67, p. 102691, 2021. [Online]. Available: https://www.sciencedirect.com/science/article/pii/S2210670720309069

[265] K. E. Zarecor, D. J. Peters, and S. Hamideh, Rural Smart Shrinkage and Perceptions of Quality of Life in the American Midwest. Cham: Springer International Publishing, 2021, pp. 395-415. [Online]. Available: https://doi.org/10.1007/978-3-030-50540-0_20

[266] I. L.A., G. T.A., G. M.P., and G. V.V, "Digital business model and smart economy sectoral development trajectories substantiation," in Internet of Things, Smart Spaces, and Next Generation Networks and Systems, vol. 10531. Springer International Publishing, 2017, pp. pp 13-28.

[267] L. Svobodová and D. Bednarska-Olejniczak, "Smart city and economy: Bibliographic coupling and co-occurrence," in Conference on e-Business, e-Services and e-Society. Springer, 2020, pp. 102-113.

[268] K. Govindan, K. M. Shankar, and D. Kannan, "Achieving sustainable development goals through identifying and analyzing barriers to industrial sharing economy: A framework development," International Journal of Production Economics, vol. 227, p. 107575, 2020.
[Online]. Available: https://www.sciencedirect.com/science/article/pii/ S0925527319304177

[269] S. S. Kamble, A. Gunasekaran, A. Ghadge, and R. Raut, "A performance measurement system for industry 4.0 enabled smart manufacturing system in smmes- a review and empirical investigation," International Journal of Production Economics, vol 229, p. 107853, 2020. [Online]. Available: http://www.sciencedirect. com/science/article/pii/S0925527320302176

[270] A. Chaudhuri, "Transformation with trustworthy digital: Policy desiderata for businesses in post covid-19 world," EDPACS, vol. 63, no. 1, pp. 1-8, 2021.

[271] M. Mkansi, "E-business adoption costs and strategies for retail micro businesses," Electronic Commerce Research, pp. 1-41, 2021

[272] L. Cao, "Ai in fintech: A research agenda," arXiv preprint arXiv:2007.12681, 2020

[273] X. Wang and F. Xu, "A theory of fintech and trade finance," SSRN, 2021. [Online]. Available: https://ssrn.com/abstract $=3777250$

[274] J. Lee, Y. C. Lee, and J. T. Kim, "Migration from the traditional to the smart factory in the die-casting industry: Novel process data acquisition and fault detection based on artificial neural network," Journal of Materials Processing Technology, vol. 290, p. 116972, 2021. [Online]. Available: https://www.sciencedirect.com/science/article/pii/ S0924013620303939

[275] B. I. Oladapo, S. O. Ismail, T. D. Afolalu, D. B. Olawade, and M. Zahedi, "Review on 3d printing: Fight against covid-19," Materials chemistry and physics, vol. 258, p. 123943, 2021.

[276] S. E. Starnawska, "Sustainability in the banking industry through technological transformation," in The Palgrave Handbook of Corporate Sustainability in the Digital Era. Springer, 2020, pp. 429-453.

[277] Y. Ren, R. Xie, F. R. Yu, T. Huang, and Y. Liu, "Potential identity resolution systems for the industrial internet of things: A survey," IEEE Communications Surveys Tutorials, vol. 23, no. 1, pp. 391-430, 2021.

[278] F. Strozzi, C. Colicchia, A. Creazza, and C. Noè, "Literature review on the 'smart factory' concept using bibliometric tools," International Journal of Production Research, vol. 55, no. 22, pp. 6572-6591, 2017. [Online]. Available: https://doi.org/10.1080/00207543.2017.1326643

[279] M. Resman, M. Turk, and N. Herakovič, "Methodology for planning smart factory," Procedia CIRP, vol. 97, pp. 401-406, 2021, 8th CIRP Conference of Assembly Technology and Systems. [Online]. Available: https://www.sciencedirect.com/science/article/pii/S2212827120314803

[280] G. Goh, S. Sing, and W. Yeong, "A review on machine learning in 3d printing: Applications, potential, and challenges," Artificial Intelligence Review, vol. 54, no. 1, pp. 63-94, 2021.

[281] F. Sgarbossa, M. Peron, F. Lolli, and E. Balugani, "Conventional or additive manufacturing for spare parts management: An extensive comparison for poisson demand," International Journal of Production Economics, vol. 233, p. 107993, 2021. [Online]. Available: https: //www.sciencedirect.com/science/article/pii/S092552732030342X

[282] H. Jiang, "Smart urban governance in the 'smart' era: Why is it urgently needed?" Cities, p. 103004, 2020. [Online]. Available: http://www.sciencedirect.com/science/article/pii/S0264275120313524

[283] S. Barns, "Smart cities and urban data platforms: Designing interfaces for smart governance," City, Culture and Society, vol. 12, pp. 5 12, 2018, innovation and identity in next generation smart cities. [Online]. Available: http://www.sciencedirect.com/science/article/pii/ S1877916617302047

[284] G. V. Pereira, P. Parycek, E. Falco, and R. Kleinhans, "Smart governance in the context of smart cities: A literature review," Information Polity, vol. 23, no. 2, 2018.

[285] S. Alawadhi and H. J. Scholl, "Smart governance: A cross-case analysis of smart city initiatives," in 2016 49th Hawaii International Conference on System Sciences (HICSS), 2016, pp. 2953-2963.

[286] J. Rose, J. S. Persson, and L. T. Heeager, "How e-government managers prioritise rival value positions: The efficiency imperative," Information polity, vol. 20, no. 1, pp. 35-59, 2015

[287] P. Kulkarni and K. Akhilesh, "Big data analytics as an enabler in smart governance for the future smart cities," in Smart Technologies. Springer, 2020, pp. 53-65.

[288] S. Saharan, N. Kumar, and S. Bawa, "An efficient smart parking pricing system for smart city environment: A machine-learning based approach," Future Generation Computer Systems, vol. 106, pp. $622-$ 640, 2020. [Online]. Available: http://www.sciencedirect.com/science/ article/pii/S0167739X19322496

[289] J. Ristvej, M. Lacinák, and R. Ondrejka, "On smart city and safe city concepts," Mobile Networks and Applications, vol. 25, no. 3, pp. 836$845,2020$. 
[290] T. Rademacher, Artificial Intelligence and Law Enforcement. Cham: Springer International Publishing, 2020, pp. 225-254. [Online]. Available: https://doi.org/10.1007/978-3-030-32361-5_10

[291] M. Helmold, "Total revenue management (trm)," in Total Revenue Management (TRM). Springer, 2020, pp. 1-12.

[292] F. de Haan and V. Butot, Finding Safety in the Smart City: A Discourse Analysis with Strategic Implications. Cham: Springer International Publishing, 2021, pp. 225-242. [Online]. Available: https://doi.org/10.1007/978-3-030-42523-4_16

[293] A. Muhammad, M. Asad, and A. R. Javed, "Robust early stage botnet detection using machine learning," in 2020 International Conference on Cyber Warfare and Security (ICCWS). IEEE, 2020, pp. 1-6.

[294] W. S. Saif, M. A. Esmail, A. M. Ragheb, T. A. Alshawi, and S. A. Alshebeili, "Machine learning techniques for optical performance monitoring and modulation format identification: A survey," IEEE Communications Surveys Tutorials, vol. 22, no. 4, pp. 2839- 2882 , 2020.

[295] S. S. Govada, T. Rodgers, L. Cheng, and H. Chung, "Smart environment for smart and sustainable hong kong," in Smart Environment for Smart Cities. Springer, 2020, pp. 57-90.

[296] P. Chithaluru, F. Al-Turjman, M. Kumar, and T. Stephan, "I-areor: An energy-balanced clustering protocol for implementing green iot in smart cities," Sustainable Cities and Society, p. 102254, 2020.

[297] M. S. Aslam, A. Khan, A. Atif, S. A. Hassan, A. Mahmood, H. K. Qureshi, and M. Gidlund, "Exploring multi-hop lora for green smart cities," IEEE Network, vol. 34, no. 2, pp. 225-231, 2020.

[298] W. Cao, J. Zhang, C. Cai, Q. Chen, Y. Zhao, Y. Lou, W. Jiang, and G. Gui, "Cnn-based intelligent safety surveillance in green iot applications," China Communications, vol. 18, no. 1, pp. 108-119, 2021.

[299] J. Zhang, W. Yue, P. Fan, and J. Gao, "Measuring the accessibility of public green spaces in urban areas using web map services," Applied Geography, vol. 126, p. 102381, 2021. [Online]. Available: https://www.sciencedirect.com/science/article/pii/S0143622820314806

[300] X. Liu and N. Ansari, "Toward green iot: Energy solutions and key challenges," IEEE Communications Magazine, vol. 57, no. 3, pp. 104110,2019

[301] M. A. Ferrag, L. Shu, X. Yang, A. Derhab, and L. Maglaras, "Security and privacy for green iot-based agriculture: Review, blockchain solutions, and challenges," IEEE Access, vol. 8, pp. 32 031-32 053, 2020.

[302] S. Kumar, D. Yadav, H. Gupta, O. P. Verma, I. A. Ansari, and C. W. Ahn, "A novel yolov3 algorithm-based deep learning approach for waste segregation: Towards smart waste management," Electronics, vol. 10, no. 1, 2021. [Online]. Available: https: //www.mdpi.com/2079-9292/10/1/14

[303] M. A. M. Albreem, A. A. El-Saleh, M. Isa, W. Salah, M. Jusoh, M. M. Azizan, and A. Ali, "Green internet of things (iot): An overview," in 2017 IEEE 4th International Conference on Smart Instrumentation, Measurement and Application (ICSIMA), 2017, pp. 1-6.

[304] S. A. Hamad, Q. Z. Sheng, W. E. Zhang, and S. Nepal, "Realizing an internet of secure things: A survey on issues and enabling technologies," IEEE Communications Surveys Tutorials, vol. 22, no. 2, pp. 1372-1391, 2020.

[305] Q. Chen, L. Wang, P. Chen, and G. Chen, "Optimization of component elements in integrated coding systems for green communications: A survey," IEEE Communications Surveys Tutorials, vol. 21, no. 3, pp. $2977-2999,2019$.

[306] K. Pardini, J. J. Rodrigues, O. Diallo, A. K. Das, V. H. C. de Albuquerque, and S. A. Kozlov, "A smart waste management solution geared towards citizens," Sensors, vol. 20, no. 8, p. 2380, 2020.

[307] A. Curtis, B. Küppers, S. Möllnitz, K. Khodier, and R. Sarc, "Real time material flow monitoring in mechanical waste processing and the relevance of fluctuations," Waste Management, vol. 120, pp. 687-697, 2021. [Online]. Available: https://www.sciencedirect.com/ science/article/pii/S0956053X20306085

[308] T. Alam, M. A. Khan, N. K. Gharaibeh, and M. K. Gharaibeh, Big Data for Smart Cities: A Case Study of NEOM City, Saudi Arabia. Cham: Springer International Publishing, 2021, pp. 215-230. [Online]. Available: https://doi.org/10.1007/978-3-030-60922-1_11

[309] E. E. Khalil, "12 - distributed energy in smart cities and the infrastructure," in Solving Urban Infrastructure Problems Using Smart City Technologies, J. R. Vacca, Ed. Elsevier, 2021, pp. 249-268. [Online]. Available: https://www.sciencedirect.com/science/article/pii/ B9780128168165000127

[310] M. Tharwat and A. Khattab, Clustering Techniques for Smart Cities: An Artificial Intelligence Perspective. Cham: Springer
International Publishing, 2021, pp. 113-134. [Online]. Available: https://doi.org/10.1007/978-3-030-60922-1_6

[311] C. Konstantinou, "Towards a secure and resilient all-renewable energy grid for smart cities," IEEE Consumer Electronics Magazine, pp. 1-1, 2021.

[312] T. Ahmad, D. Zhang, C. Huang, H. Zhang, N. Dai, Y. Song, and $\mathrm{H}$. Chen, "Artificial intelligence in sustainable energy industry: Status quo, challenges and opportunities," Journal of Cleaner Production, vol. 289, p. 125834, 2021. [Online]. Available: https: //www.sciencedirect.com/science/article/pii/S0959652621000548

[313] M. M. Alam, H. Malik, M. I. Khan, T. Pardy, A. Kuusik, and Y. Le Moullec, "A survey on the roles of communication technologies in iotbased personalized healthcare applications," IEEE Access, vol. 6, pp. 36611-36631, 2018. 